TRANSACTIONS OF THE

AMERICAN MATHEMATICAL SOCIETY

Volume 357, Number 2, Pages 571-615

S 0002-9947(04)03685-2

Article electronically published on September 23, 2004

\title{
ANALYSIS ON PRODUCTS OF FRACTALS
}

\author{
ROBERT S. STRICHARTZ
}

\begin{abstract}
For a class of post-critically finite (p.c.f.) fractals, which includes the Sierpinski gasket (SG), there is a satisfactory theory of analysis due to Kigami, including energy, harmonic functions and Laplacians. In particular, the Laplacian coincides with the generator of a stochastic process constructed independently by probabilistic methods. The probabilistic method is also available for non-p.c.f. fractals such as the Sierpinski carpet. In this paper we show how to extend Kigami's construction to products of p.c.f. fractals. Since the products are not themselves p.c.f., this gives the first glimpse of what the analytic theory could accomplish in the non-p.c.f. setting. There are some important differences that arise in this setting. It is no longer true that points have positive capacity, so functions of finite energy are not necessarily continuous. Also the boundary of the fractal is no longer finite, so boundary conditions need to be dealt with in a more involved manner. All in all, the theory resembles PDE theory while in the p.c.f. case it is much closer to ODE theory.
\end{abstract}

\section{INTRODUCTION}

Euclidean space may be constructed as a product of lines, and analysis on Euclidean space may be obtained by appropriately transporting corresponding concepts from the line. This will be our model for creating analysis on a product of fractals. Because products of post-critically finite (p.c.f.) fractals are not p.c.f., this enables us to construct an analytic theory on an entirely different kind of fractal. Indeed, the "differential equations" on p.c.f. fractals such as the Sierpinski gasket (SG) have much in common with ODE's on the line or interval. We will see that the analogous theory on products resembles PDE theory. Of course this is not surprising. The point is that by exploiting the product structure we are able to obtain significant results rather easily. We hope that this will serve as a model for what we can expect, and not expect, from analysis on a wider class of fractals.

The analytic theory on p.c.f. fractals was developed by Kigami Ki1 - Ki4 following the work of several probabilists who constructed stochastic processes analogous to Brownian motion, thus obtaining a Laplacian indirectly as the generator of the process. See the book of Barlow $[\mathrm{Ba}$ for an account of this development. At present, both points of view have important consequences for the theory. Although we emphasize the analytic development, we will need to use heat kernel estimates,

Received by the editors July 8, 2003.

2000 Mathematics Subject Classification. Primary 31C45, 28A80.

The author's research was supported in part by the National Science Foundation, grant DMS0140194 . 
which are obtainable only via probability theory. The expository article [S3] explains some of the goals of the theory in comparision with analysis on manifolds. We refer the reader to the book $\mathrm{Ki3}$, for complete details.

The four main concepts in analysis on p.c.f. fractals are: energy, harmonic functions, Laplacian, and resistance metric. The energy form $\mathcal{E}(u, v)$, the analog of

$\int_{0}^{1} u^{\prime}(x) v^{\prime}(x) d x$ on the unit interval, is constructed as the limit of renormalized graph energies on a sequence of graphs $\left\{\Gamma_{m}\right\}$ approximating the fractal. This approach is feasible because points have positive capacity, which implies that finite energy functions are continuous. This approach is not feasible in the product setting, for example in the case of the square. However, in Section 2 we are able to give several equivalent and natural definitions of $\mathcal{E}(u, v)$ in the product setting. A harmonic function is defined to be an energy minimizer subject to boundary conditions. In the p.c.f. case the boundary is finite, so the space of harmonic functions is finite dimensional, and it is best to think of harmonic functions as analogs of linear functions on the interval. In the product setting, the space of harmonic functions is infinite dimensional. We give elementary properties of harmonic functions in Section 3, but postpone until Section 8 more precise results. For technical reasons we introduce the more restricted class of pluriharmonic functions, that form a finite dimensional space, and may be computed precisely.

When we define a Laplacian $\Delta$ in Section 4 we will show that harmonic functions coincide with solutions of $\Delta h=0$. We use a weak form to define $\Delta$, essentially

$$
-\mathcal{E}(u, v)=\int(\Delta u) v d \mu
$$

if $v$ vanishes on the boundary. In the p.c.f. case it is possible to identify the $L^{2}$ domain of $\Delta$ with a natural Sobolev space, but this is not true in the product setting. For example, there are many harmonic functions in the square that belong to the Sobolev space $H^{1}$ but not $H^{2}$. The key idea is that we need to have boundary values in the appropriate boundary Sobolev spaces. In the p.c.f. we get this for free because the boundary is so small. It is not until Section 7 that we are able to present a full characterization of the appropriate Sobolev space of functions with Laplacians in $L^{2}$. For functions in this Sobolev space we are able to prove some of the basic results known in the p.c.f. case, including existence of normal derivatives on the boundary and the Gauss-Green formula. We are also able to prove $L^{2}$ boundedness of Riesz transforms and a Fubini-type characterization of the Sobolev norm, results which have no analog in the p.c.f. setting. The Riesz transforms are clearly natural examples of the analog of Calderon-Zygmund operators in this setting; at present there are no natural candidates for Calderon-Zygmund operators on p.c.f. fractals, other than the Hilbert transform on the interval.

There is no analog of the resistance metric in the product setting; we do not see any way to relate resistance networks to the structure of these fractals. Instead, we cobble together resistance metrics on each of the factors to define a metric on the product, essentially mimicking the Pythagorean theorem in Euclidean space (more precisely a Finsler metric construction). In Section 6 we show easily that heat kernel estimates extend from the factors to the product, using this metric. Heat kernel estimates have many pleasant consequences, and we use them in key ways in the rest of the paper. 
Because the boundary creates difficulties, we find it convenient to work in a "cover space" that has no boundary. In the p.c.f. setting, we construct the double cover by taking two copies of the fractal and identifying common boundary points. In [S6] we showed that in the case of SG the double cover fits into a general framework of fractafolds, where every point has a neighborhood isomorphic to a neighborhood in the original fractal. While this is not necessarily the case for all p.c.f. fractals, it is still fairly easy to extend all constructions from the fractal to its double cover. In particular, the eigenfunctions of the Laplacian on the double cover consist of just the odd extensions of Dirichlet eigenfunctions and the even extensions of Neumann eigenfunctions on the fractal. In the product setting we simply take the product of the double covers of the factors. For the case of the square this produces a quadruple cover by a 2 -torus. In Section 5 we show how to characterize the domain of the energy form and the $L^{2}$ domain of the Laplacian on the cover space of a product fractal in terms of Sobolev spaces defined by eigenfunction expansions. This enables us to prove a number of important properties. The issue of the corresponding properties on the product fractal is then reduced to an extension problem: which functions on the fractal can be extended to the cover to lie in the appropriate Sobolev space? For the domain of the energy form this is trivial, since an even reflection suffices. For the $L^{2}$ domain of the Laplacian we show in Theorem 7.7 that extension is equivalent to the trace on the boundary belonging to the appropriate Sobolev space.

Section 7 deals with traces and extensions relating Sobolev spaces on $K$ and its boundary. We are able to identify the correct trace space for normal derivatives, but the analog of Theorem 7.7 remains a conjecture. Section 8 deals with representation formulas for harmonic functions, including an analog of the Poisson integral. In Section 9 we briefly discuss pointwise formulas for energy and Laplacian that are valid under suitable regularity assumptions. In Section 10 we give characterizations of Sobolev spaces of small order, both in the p.c.f. and the product setting. These results seem rather generic, and should be valid in any context in which the appropriate heat kernel estimates are true. We conclude with Section 11 in which we discuss hypoellipticity properties. We show that harmonic functions are automatically smooth, and we conjecture that $u$ must be smooth on an open set if $\Delta u=f$ and $f$ is smooth on that open set.

The simplest example of a product fractal is the square, but it is not truly fractal, merely self-similar. All the results in this paper are already known for the square, although it may be difficult to find some of them in the literature. So perhaps the basic example to consider is $\mathrm{SG}^{2}=\mathrm{SG} \times \mathrm{SG}$. This is a self-similar fractal generated by an iterated function system (i.f.s.) of 9 mappings $\left\{F_{i}^{\prime} \times F_{j}^{\prime}\right\}$ for $i, j=1,2,3$, where $\left\{F_{i}^{\prime}\right\}$ is the i.f.s. generating SG. Let $\left\{q_{1}^{\prime}, q_{2}^{\prime}, q_{3}^{\prime}\right\}$ denote the boundary points of SG. Then the boundary of $\mathrm{SG}^{2}$ is made up of six "faces", three horizontal faces $S G \times\left\{q_{i}^{\prime}\right\}$ and three vertical faces $\left\{q_{i}^{\prime}\right\} \times \mathrm{SG}$. The 9 points $\left\{\left(q_{i}^{\prime}, q_{j}^{\prime}\right)\right\}$ make up what might be called the distinguished boundary of $\mathrm{SG}^{2}$. Horizontal and vertical faces meet at these points. The boundary forms a fractafold without boundary in the sense of [S6], but we will not use the spectral analysis of this fractafold here (just like the identification of the boundary of the square with a circle does not play a role in analysis on the square). The 9 cells $F_{i}^{\prime} \times F_{j}^{\prime}\left(\mathrm{SG}^{2}\right)=F_{i}^{\prime}(\mathrm{SG}) \times F_{j}^{\prime}(\mathrm{SG})$ of level 1 that make up $\mathrm{SG}^{2}$ intersect either at single points or on sets isomorphic to SG. In particular, $\mathrm{SG}^{2}$ is not finitely ramified: it remains connected after the removal 
of finitely many points. (In fact, it should be possible to prove that to disconnect $\mathrm{SG}^{2}$ it is necessary to remove a set of the same dimension as SG.)

The construction of $\mathrm{SG}^{2}$ naturally embeds it in $\mathbb{R}^{4}$. Of course we need to emphasize that, just as in the p.c.f. case, the analysis is based on the topology of the fractal, and not the geometry in any particular realization. In fact, SG with its resistance metric does not embed isometrically (or even quasi-isometrically) in any Euclidean space, and the same will be true for $\mathrm{SG}^{2}$ with the metric we use. Nevertheless, it would be desirable to have a geometric realization that allows us to "visualize" the fractal. The Hausdorff dimension of $\mathrm{SG}^{2}$ in its self-similar embedding in $\mathbb{R}^{4}$ is $2 \log 3 / \log 2>3$, so this precludes a quasi-isometric embedding in $\mathbb{R}^{3}$. (Note added in proof: Maria Roginskaya has shown that $\mathrm{SG}^{2}$ does not embed topologically in $\mathbb{R}^{3}$.)

A simpler product, $[0,1] \times \mathrm{SG}$, is easy to visualize (a vertical stack of SG's), but because of the mismatch of scaling factors we are not able to include this example in many of our theorems. In fact the full strength of our theory only applies to products with identical factors, and the scaling factors must be homogeneous throughout the fractal. Of course, it can be argued that $[0,1] \times \mathrm{SG}$ is the natural underlying space for the space-time equations on SG, such as the heat and wave equation, that have been extensively studied [DSV], CDS]. Indeed, the mismatch in scaling factors has already been identified as a cause for some of the unexpected behaviors of solutions of these equations. The product fractals discussed in this work allow a wide range of "differential operators" beside the Laplacian; future work might reveal a full "fractal PDE" theory.

A finite element method numerical algorithm for $S G^{2}$ has been worked out by Brian Bockelman, and is available at the website www.math.cornell.edu/ ${ }^{\text {stu } 28041 /}$ fem/index.htm.

We have structured the paper to add assumptions as we go along. In the early sections we show that a reasonable theory exists on a wide class of products, but in order to obtain the more precise results of the later sections we have to narrow the class of examples.

Some of our results depend on estimates for normal derivatives of the Dirichlet heat kernel on the p.c.f. factors. At present, these estimates ((7.16), (8.3) and (8.11)) have not been established on any fractals (other than the interval). We believe that (8.11) is essentially trivial, but (7.16) and (8.3) are more challenging. Numerical evidence for (7.16) in the case of the Sierpinski gasket may be found at the website www.math.cornell.edu/ $\sim$ Bengal/ and in the paper [BSSY]. Heat kernel estimates in general require probabilistic methods, which are quite different than the methods used in this paper. We hope that by exhibiting some interesting consequences of these estimates we will stimulate work on proving them.

Notation conventions. So as not to burden the reader with excessive notation, we work with prodcts of just two factors. The extension of the results to products with more than two factors should be routine. We denote by a prime anything having to do with the first factor, and by a double prime anything having to do with the second factor. Thus the product will be written $K=K^{\prime} \times K^{\prime \prime}$, a variable point in $K$ will be $x=\left(x^{\prime}, x^{\prime \prime}\right)$. Similarly $\mathcal{E}^{\prime}$ and $\Delta^{\prime}$ denote energy and Laplacian on $K^{\prime}$. This notation should be self-explanatory, and we hope it will help the reader easily grasp the meaning of all equations. We also need to distinguish between spaces and their covers, and we do this by using a tilde for the cover. Thus $\widetilde{K}^{\prime}$ and $\widetilde{K}^{\prime \prime}$ denote 
the double covers of $K^{\prime}$ and $K^{\prime \prime}$, and $\widetilde{K}=\widetilde{K}^{\prime} \times \widetilde{K}^{\prime \prime}$ is the quadruple cover of $K$. We will not put tildes over variables, but by writing $\widetilde{u}^{\prime}\left(x^{\prime}\right)$ it will be clear that we are referring to a function on $\widetilde{K}^{\prime}$.

Next we describe the basic assumptions on the factor $K^{\prime}$ (we make the same assumptions about $K^{\prime \prime}$ ): $K^{\prime}$ is a p.c.f. self-similar set with boundary $V_{0}^{\prime}$, selfsimilar measure $\mu^{\prime}$ and self-similar regular energy $\mathcal{E}^{\prime}$. For simplicty of notation we assume that $K^{\prime}$ is embedded in some Euclidean space, and $K^{\prime}$ is the invariant set for a contractive linear iterated function system (i.f.s.) $\left\{F_{i}^{\prime}\right\}_{i=1, \ldots, n_{0}^{\prime}}$. Let $q_{i}^{\prime}$ denote the fixed point of $F_{i}^{\prime}$, and let $V_{0}^{\prime}=\left\{q_{i}\right\}_{i=1, \ldots, n^{\prime}}$ for some $n^{\prime} \leq n_{0}^{\prime}$. The p.c.f. condition is that $K^{\prime}$ is connected and

$$
F_{i}^{\prime} K^{\prime} \cap F_{j}^{\prime} K^{\prime} \subseteq F_{i}^{\prime} V_{0}^{\prime} \cap F_{j}^{\prime} V_{0}^{\prime} \text { for } i \neq j .
$$

We refer to the sets $F_{i}^{\prime} K^{\prime}$ as cells of level 1 . In general we write $w^{\prime}=\left(w_{1}^{\prime}, \ldots, w_{m}^{\prime}\right)$ for a word of length $\left|w^{\prime}\right|=m$, each $w_{j}^{\prime}$ chosen from $\left\{1, \ldots, n_{0}^{\prime}\right\}$, and $F_{w^{\prime}}^{\prime}=F_{w_{1}^{\prime}}^{\prime} \circ$ $\cdots \circ F_{w_{m}^{\prime}}^{\prime}$. Then the sets $F_{w^{\prime}}^{\prime} K^{\prime}$ are called cells of level $m$. Distinct cells of a given level can intersect only at isolated points. The self-similar probability measure $\mu^{\prime}$ is determined by the choice of probability weights $\left\{\mu_{i}^{\prime}\right\}_{i=1, \ldots, n_{0}^{\prime}}$ via the identity

$$
\mu^{\prime}=\sum_{i=1}^{n_{0}^{\prime}} \mu_{i}^{\prime} \mu^{\prime} \circ\left(F_{i}^{\prime}\right)^{-1},
$$

or equivalently

$$
\int_{K^{\prime}} f d \mu^{\prime}=\sum_{i=1}^{n_{0}^{\prime}} \mu_{i}^{\prime} \int f \circ F_{i}^{\prime} d \mu^{\prime} .
$$

To define the self-similar energy we consider $K^{\prime}$ as the limit of graphs $\Gamma_{m}^{\prime}$ with vertices $V_{m}^{\prime}$ and edge relation $x^{\prime} \sim_{m} y^{\prime}$ defined inductively as follows: $\Gamma_{0}^{\prime}$ is the complete graph on $V_{0}^{\prime}$, and $V_{m}^{\prime}=\bigcup_{i=1}^{n_{0}^{\prime}} F_{i}^{\prime} V_{m-1}^{\prime}$ and $x^{\prime} \sim_{m} y^{\prime}$ if and only if $x^{\prime}$ and $y^{\prime}$ belong to the same cell $F_{w^{\prime}}^{\prime} K^{\prime}$ of level $m$. We choose positive conductances $c_{i j}^{\prime}=c_{j i}^{\prime}$ for $i \neq j, i, j=1, \ldots, n^{\prime}$. In other words $c^{\prime}$ are defined on the edges of the graph $\Gamma_{0}^{\prime}$. We may think of the reciprocals as resistances in an electrical network with vertices $V_{0}^{\prime}$. We choose also resistance renormalization factors $r_{i}^{\prime}$ for $i=1, \ldots, n_{0}^{\prime}$ satisfying $0<r_{i}^{\prime}<1$. Then we create a self-similar electrical network on $\Gamma_{m}^{\prime}$ by defining $c_{x^{\prime} y^{\prime}}^{\prime}=\left(r_{w^{\prime}}^{\prime}\right)^{-1} c_{i j}^{\prime}$ if $x^{\prime}=F_{w^{\prime}}^{\prime} q_{i}^{\prime}$ and $y^{\prime}=F_{w^{\prime}}^{\prime} q_{j}^{\prime}$, where $r_{w^{\prime}}^{\prime}=r_{w_{1}^{\prime}}^{\prime} \cdots r_{w_{m}^{\prime}}^{\prime}$. Note that the condition $r_{i}^{\prime}<1$ means that the resistances of the edges tend to zero as $m \rightarrow \infty$. The energy $\mathcal{E}_{m}^{\prime}\left(u^{\prime}, v^{\prime}\right)$ for $u^{\prime}, v^{\prime}$ functions on $V_{m}^{\prime}$ is defined by

$$
\mathcal{E}_{m}^{\prime}\left(u^{\prime}, v^{\prime}\right)=\sum_{x^{\prime} \sim m} c_{x^{\prime} y^{\prime}}\left(u^{\prime}\left(x^{\prime}\right)-u^{\prime}\left(y^{\prime}\right)\right)\left(v^{\prime}\left(x^{\prime}\right)-v^{\prime}\left(y^{\prime}\right)\right) .
$$

Note that we also have

$$
\mathcal{E}_{m}^{\prime}\left(u^{\prime}, v^{\prime}\right)=\sum_{\left|w^{\prime}\right|=m}\left(r_{w}^{\prime}\right)^{-1} \mathcal{E}_{0}^{\prime}\left(u^{\prime} \circ F_{w}^{\prime}, v^{\prime} \circ F_{w}^{\prime}\right) .
$$

This alone is not enough to enable us to pass to the limit as $m \rightarrow \infty$. We need one more very restrictive compatibility condition:

$$
\mathcal{E}_{1}^{\prime}\left(\bar{u}^{\prime}, \bar{u}^{\prime}\right)=\mathcal{E}_{0}^{\prime}\left(u^{\prime}, u^{\prime}\right),
$$


where $\bar{u}^{\prime}$ is chosen among all extensions of $u^{\prime}$ to $V_{1}^{\prime}$ so as to minimize $\mathcal{E}_{1}^{\prime}$ (this is called the harmonic extension). This places rather complicated nonlinear constraints on the initial conductances and resistance renormalization factors. Questions about existence and uniqueness of such energies are still not completely resolved, but it suffices for our purposes to note that there are many explicit examples known, including the unit interval $I$ (both $r_{i}^{\prime}=1 / 2$ ) and SG (all $c_{i j}^{\prime}=1$ and all $r_{i}^{\prime}=3 / 5$ ).

It follows easily from $(1.7)$ that $\mathcal{E}_{m}^{\prime}\left(u^{\prime}, u^{\prime}\right)$ is a monotone increasing function for any $u^{\prime}$ defined on $K^{\prime}$, hence

$$
\mathcal{E}^{\prime}\left(u^{\prime}, u^{\prime}\right)=\lim _{m \rightarrow \infty} \mathcal{E}_{m}^{\prime}\left(u^{\prime}, u^{\prime}\right)
$$

is well defined as an extended real number, and we define dom $\mathcal{E}^{\prime}$ to be the functions for which the limit is finite. The basic facts are that dom $\mathcal{E}^{\prime}$ is a dense subspace of $C\left(K^{\prime}\right)$, and modulo constants it forms a Hilbert space with norm $\mathcal{E}^{\prime}\left(u^{\prime}, u^{\prime}\right)^{1 / 2}$. For $u^{\prime}, v^{\prime} \in \operatorname{dom} \mathcal{E}^{\prime}$,

$$
\mathcal{E}^{\prime}\left(u^{\prime}, v^{\prime}\right)=\lim _{m \rightarrow \infty} \mathcal{E}_{m}^{\prime}\left(u^{\prime}, v^{\prime}\right)
$$

is well defined and gives the inner product. There is an $n^{\prime}$-dimensional space $\mathcal{H}_{0}^{\prime}$ of harmonic functions obtained by assigning values in $V_{0}^{\prime}$, taking the minimum energy extension at each level, and extending from $V_{*}^{\prime}=\bigcup_{m=0}^{\infty} V_{m}^{\prime}$ to $K^{\prime}$ by continuity. For harmonic functions, $\mathcal{E}_{m}^{\prime}\left(u^{\prime}, u^{\prime}\right)$ is independent of $m$ (in fact $\mathcal{E}_{m}^{\prime}\left(u^{\prime}, v^{\prime}\right)$ is independent of $m$ if just $u^{\prime}$ is harmonic). The self-similarity of the energy $\mathcal{E}^{\prime}$ may be expressed as

$$
\mathcal{E}^{\prime}\left(u^{\prime}, v^{\prime}\right)=\sum_{i=1}^{n_{0}^{\prime}}\left(r_{i}^{\prime}\right)^{-1} \mathcal{E}^{\prime}\left(u^{\prime} \circ F_{i}^{\prime}, v^{\prime} \circ F_{i}^{\prime}\right),
$$

or more generally

$$
\mathcal{E}^{\prime}\left(u^{\prime}, v^{\prime}\right)=\sum_{\left|w^{\prime}\right|=m}\left(r_{w^{\prime}}^{\prime}\right)^{-1} \mathcal{E}^{\prime}\left(u^{\prime} \circ F_{w^{\prime}}^{\prime}, u^{\prime} \circ F_{w^{\prime}}^{\prime}\right) .
$$

Given the energy $\mathcal{E}^{\prime}$, we define a natural metric, called the effective resistance metric on $K^{\prime}$, by

$$
d\left(x^{\prime}, y^{\prime}\right)=\left(\min \left\{\mathcal{E}(u, u): u\left(x^{\prime}\right)=0 \text { and } u\left(y^{\prime}\right)=1\right\}\right)^{-1} .
$$

An important observation is that this metric has nothing to do with the Euclidean metric of the ambient space where $K^{\prime}$ is embedded. The construction of $\mathcal{E}^{\prime}$ in fact only uses the topology of $K^{\prime}$, and it can be shown that the effective resistance metric yields the same topology. In most examples, the fractal $K^{\prime}$ with the effective resistance metric will not embed isometrically (or even quasi-isometrically) in any Euclidean space. The effective resistance metric is not exactly self-similar, but is approximately self-similar: each cell $F_{w^{\prime}}^{\prime} K^{\prime}$ has diameter on the order of $r_{w^{\prime}}^{\prime}$.

Given the energy $\mathcal{E}^{\prime}$ and measure $\mu^{\prime}$, we define a Laplacian $\Delta^{\prime}$ with domain $\operatorname{dom} \Delta^{\prime}$ as follows: $u^{\prime} \in \operatorname{dom} \Delta^{\prime}$ with $\Delta^{\prime} u^{\prime}=f^{\prime}$ for $u^{\prime} \in \operatorname{dom} \mathcal{E}^{\prime}$ and $f^{\prime} \in C\left(K^{\prime}\right)$ provided

$$
-\mathcal{E}^{\prime}\left(u^{\prime}, v^{\prime}\right)=\int_{K^{\prime}} f^{\prime} v^{\prime} d \mu^{\prime} \text { for all } v^{\prime} \in \operatorname{dom}_{0} \mathcal{E}
$$


where $\operatorname{dom}_{0} \mathcal{E}^{\prime}$ denotes the subspace of dom $\mathcal{E}^{\prime}$ of functions vanishing on the boundary $V_{0}^{\prime}$. There is also an equivalent pointwise formula

$$
\Delta^{\prime} u^{\prime}=\lim _{m \rightarrow \infty} \Delta_{m}^{\prime} u^{\prime}
$$

for the graph Laplacian

$$
\Delta_{m}^{\prime} u^{\prime}\left(x^{\prime}\right)=\left(\int \psi_{x^{\prime}}^{(m)} d \mu^{\prime}\right)^{-1} \sum_{y^{\prime} \sim m} c_{x^{\prime}}^{\prime} c_{x^{\prime} y^{\prime}}\left(u^{\prime}\left(y^{\prime}\right)-u^{\prime}\left(x^{\prime}\right)\right)
$$

where $\psi_{x^{\prime}}^{(m)}$ is the piecewise harmonic spline of level $m$ equal to 1 at $x^{\prime}$ and 0 at all other vertices in $V_{m}^{\prime}$. The limit (1.14) must be uniform (note that $\Delta_{m}^{\prime} u^{\prime}$ is not defined on $V_{0}^{\prime}$, however) in a suitable sense. (On SG (1.15) takes the simple form

$$
\Delta_{m}^{\prime} u^{\prime}\left(x^{\prime}\right)=\left(\frac{3}{2}\right) 5^{m} \sum_{y^{\prime} \sim_{m} x^{\prime}}\left(u^{\prime}\left(y^{\prime}\right)-u^{\prime}\left(x^{\prime}\right)\right)
$$

for $x^{\prime} \in V_{m}^{\prime} \backslash V_{0}^{\prime}$, and there are exactly four terms in the sum (1.16).) We also define $\operatorname{dom}_{L^{2}} \Delta^{\prime}$ by requiring (1.13) to hold for some $f^{\prime} \in L^{2}$. The equivalent pointwise formula would require the limit (1.14) to hold in a suitable $L^{2}$ sense. (This equivalence is not explictly stated in the literature, but should be fairly straightforward to derive.) The Laplacian satisfies the scaling identity

$$
\Delta^{\prime}\left(u^{\prime} \circ F_{w^{\prime}}^{\prime}\right)=r_{w} \mu_{w}\left(\Delta u^{\prime}\right) \circ F_{w^{\prime}}^{\prime} .
$$

Another important concept is the normal derivative at boundary points. The definition only depends on $\mathcal{E}^{\prime}$ :

$$
\partial_{n} u^{\prime}\left(q_{i}^{\prime}\right)=\lim _{m \rightarrow \infty}\left(r_{i}^{\prime}\right)^{-m} \sum_{y^{\prime} \sim q_{m} q_{i}^{\prime}}\left(u^{\prime}\left(q_{i}^{\prime}\right)-u^{\prime}\left(y^{\prime}\right)\right)
$$

if the limit exists. But the theorem guaranteeing existence requires that $u^{\prime} \in$ $\operatorname{dom} \Delta^{\prime}\left(\right.$ or $\left.\operatorname{dom}_{L^{q}} \Delta^{\prime}\right)$. (Note that this will hold for any choice of weights $\left\{\mu_{i}^{\prime}\right\}$ defining $\mu$, even though these weights do not enter into (1.18).) We can then state two forms of the Gauss-Green formula:

$$
-\mathcal{E}^{\prime}\left(u^{\prime}, v^{\prime}\right)=\int_{K^{\prime}}\left(\Delta^{\prime} u^{\prime}\right) v^{\prime} d \mu-\sum_{V_{0}^{\prime}}\left(\partial_{n} u^{\prime}\right) v^{\prime}
$$

for $u^{\prime} \in \operatorname{dom}_{L^{2}} \Delta^{\prime}$ and $v^{\prime} \in \operatorname{dom} \mathcal{E}^{\prime}$, and

$$
\int_{K^{\prime}}\left(\left(\Delta^{\prime} u^{\prime}\right) v^{\prime}-u^{\prime} \Delta^{\prime} v^{\prime}\right) d \mu^{\prime}=\sum_{V_{0}^{\prime}}\left(\left(\partial_{n} u^{\prime}\right) v^{\prime}-u^{\prime} \partial_{n} v^{\prime}\right)
$$

for $u^{\prime}, v^{\prime} \in \operatorname{dom}_{L^{2}} \Delta^{\prime}$. These formulas also localize to any cell.

\section{ENERGY}

Suppose we are given energy forms $\mathcal{E}^{\prime}$ on $K^{\prime}$ and $\mathcal{E}^{\prime \prime}$ on $K^{\prime \prime}$, and probability measures $\mu^{\prime}$ on $K^{\prime}$ and $\mu^{\prime \prime}$ on $K^{\prime \prime}$. We can then construct an energy form $\mathcal{E}$ on $K$ with domain $\operatorname{dom} \mathcal{E}$ that we can represent schematically as $\mathcal{E}^{\prime} \times \mu^{\prime \prime}+\mu^{\prime} \times \mathcal{E}^{\prime \prime}$ as follows.

Definition 2.1. For $u \in L^{2}\left(K, \mu^{\prime} \times \mu^{\prime \prime}\right)$ let

$$
\mathcal{E}(u, u)=\int_{K^{\prime \prime}} \mathcal{E}^{\prime}\left(u\left(\cdot, x^{\prime \prime}\right), u\left(\cdot, x^{\prime \prime}\right)\right) d \mu^{\prime \prime}\left(x^{\prime \prime}\right)+\int_{K^{\prime}} \mathcal{E}^{\prime \prime}\left(u\left(x^{\prime}, \cdot\right), u\left(x^{\prime}, \cdot\right)\right) d \mu^{\prime}\left(x^{\prime}\right) .
$$


We say $u \in \operatorname{dom} \mathcal{E}$ if $\mathcal{E}(u, u)<\infty$. For $u$ and $v$ in $\operatorname{dom} \mathcal{E}$ we let

$$
\mathcal{E}(u, v)=\int_{K^{\prime \prime}} \mathcal{E}^{\prime}\left(u\left(\cdot, x^{\prime \prime}\right), v\left(\cdot, x^{\prime \prime}\right)\right) d \mu^{\prime \prime}\left(x^{\prime \prime}\right)+\int_{K^{\prime}} \mathcal{E}^{\prime \prime}\left(u\left(x^{\prime}, \cdot\right), v\left(x^{\prime}, \cdot\right)\right) d \mu^{\prime}\left(x^{\prime}\right) .
$$

If $C=F_{w} K$ is any cell, then we define similarly $\mathcal{E}_{C}(u, u)$, the energy restricted to $C$. We do the same for any simple subset of $K$, defined to be a finite union of cells.

We assume that $\mathcal{E}^{\prime}, \mu^{\prime}, \mathcal{E}^{\prime \prime}, \mu^{\prime \prime}$ are self-similar with weights $r_{i}^{\prime}, \mu_{i}^{\prime}$ and $r_{j}^{\prime \prime}, \mu_{j}^{\prime \prime}$ for mappings $F_{i}^{\prime}$ and $F_{j}^{\prime \prime}$. It is only in very special cases that $\mathcal{E}$ is self-similar for the mappings $F_{i}^{\prime} \otimes F_{j}^{\prime \prime}$.

Lemma 2.2. Suppose there is a positive constant $b$ such that $r_{i}^{\prime} \mu_{i}^{\prime}=b^{-1}$ and $r_{j}^{\prime \prime} \mu_{j}^{\prime \prime}=b^{-1}$ for all $i$ and $j$. Then $\mathcal{E}$ is self-similar with weights $b r_{i}^{\prime} r_{j}^{\prime \prime}$ for the mapping $F_{i}^{\prime} \otimes F_{j}^{\prime \prime}$, namely

$$
\mathcal{E}(u, v)=\sum_{i=1}^{n^{\prime}} \sum_{j=1}^{n^{\prime \prime}}\left(b r_{i}^{\prime} r_{j}^{\prime \prime}\right)^{-1} \mathcal{E}\left(u \circ\left(F_{i}^{\prime} \otimes F_{j}^{\prime \prime}\right), v \circ\left(F_{i}^{\prime} \otimes F_{j}^{\prime \prime}\right)\right) .
$$

Proof. The self-similarity properties of $\mathcal{E}^{\prime}$ and $\mu^{\prime \prime}$ imply

$$
\begin{aligned}
\int_{K^{\prime \prime}} & \mathcal{E}^{\prime}\left(u\left(x^{\prime}, x^{\prime \prime}\right), v\left(x^{\prime}, x^{\prime \prime}\right)\right) d \mu^{\prime \prime}\left(x^{\prime \prime}\right) \\
= & \sum_{i=1}^{n^{\prime}} \sum_{j=1}^{n^{\prime \prime}}\left(\mu_{j}^{\prime \prime} / r_{i}^{\prime}\right) \mathcal{E}^{\prime}\left(u\left(F_{i}^{\prime} x^{\prime}, F_{j}^{\prime \prime} x^{\prime \prime}\right), u\left(F_{i}^{\prime} x^{\prime}, F_{j}^{\prime \prime} x^{\prime \prime}\right)\right) d \mu^{\prime \prime}\left(x^{\prime \prime}\right) .
\end{aligned}
$$

We obtain a similar expression with a factor of $\left(\mu_{i}^{\prime} / r_{j}^{\prime \prime}\right)$ for the second term in (2.2). But our assumptions imply $r_{i}^{\prime} / \mu_{j}^{\prime \prime}=r_{j}^{\prime \prime} / \mu_{i}^{\prime}=b r_{i}^{\prime} r_{j}^{\prime \prime}$.

For the case of the square, $r_{i}^{\prime}=\mu_{i}^{\prime}=1 / 2$ and $b=4$, so $b r_{i}^{\prime} r_{j}^{\prime \prime}=1$ and $(2.3)$ is a special case of the conformal invariance of energy. For the case of $\mathrm{SG}^{2}, r_{i}^{\prime}=3 / 5$ and $\mu_{i}^{\prime}=1 / 3$ so $b=5$ and $b r_{i}^{\prime} r_{j}^{\prime \prime}=9 / 5$. In the symmetric case $K^{\prime}=K^{\prime \prime}$, if we are given a self-similar energy $\mathcal{E}^{\prime}=\mathcal{E}^{\prime \prime}$ with weights $r_{i}^{\prime}$, we can always choose a self-similar measure $\mu^{\prime}=\mu^{\prime \prime}$ to satisfy the hypotheses of Lemma 2.2 , namely take $\mu_{i}^{\prime}=\left(b r_{i}^{\prime}\right)^{-1}$ for $b=\sum r_{i}^{-1}$. Note, however, that this differs from what is usually considered the natural choice $\left(\mu_{i}^{\prime}=\left(r_{i}^{\prime}\right)^{\alpha}\right.$ where $\left.\sum\left(r_{i}^{\prime}\right)^{\alpha}=1\right)$, unless all the $r_{i}$ weights are equal.

It is easy to construct functions in $\operatorname{dom} \mathcal{E}$ by taking tensor products of dom $\mathcal{E}^{\prime}$ and dom $\mathcal{E}^{\prime \prime}$. In the next definition we single out a special case of such a construction that will be very useful.

Definition 2.3. A function on $K$ is called pluriharmonic if it satisfies both $\Delta_{x^{\prime}}^{\prime} u\left(x^{\prime}, x^{\prime \prime}\right)=0$ and $\Delta_{x^{\prime \prime}}^{\prime \prime} u\left(x^{\prime}, x^{\prime \prime}\right)=0$. The space of pluriharmonic functions, denoted $\mathrm{PH}$, coincides with $\mathcal{H}_{0}^{\prime} \otimes \mathcal{H}_{0}^{\prime \prime}$, with dimension $n^{\prime} n^{\prime \prime}$, and natural basis

$$
h_{i j}\left(x^{\prime}, x^{\prime \prime}\right)=h_{i}^{\prime}\left(x^{\prime}\right) h_{j}^{\prime \prime}\left(x^{\prime \prime}\right) .
$$

A function $u \in \mathrm{PH}$ is uniquely determined by its values on the distinguished boundary $V_{0}^{\prime} \times V_{0}^{\prime \prime}$ by

$$
u=\sum_{i=1}^{n^{\prime}} \sum_{j=1}^{n^{\prime \prime}} u\left(q_{i}^{\prime}, q_{j}^{\prime \prime}\right) h_{i j}
$$

A function is called piecewise pluriharmonic of level $m\left(\mathrm{PPH}_{m}\right)$ if $u$ is continuous and $u\left(F_{w^{\prime}}^{\prime} x^{\prime}, F_{w^{\prime \prime}}^{\prime \prime} x^{\prime \prime}\right) \in \mathrm{PH}$ for all words $w^{\prime}$ and $w^{\prime \prime}$ of length $m$. A function in 


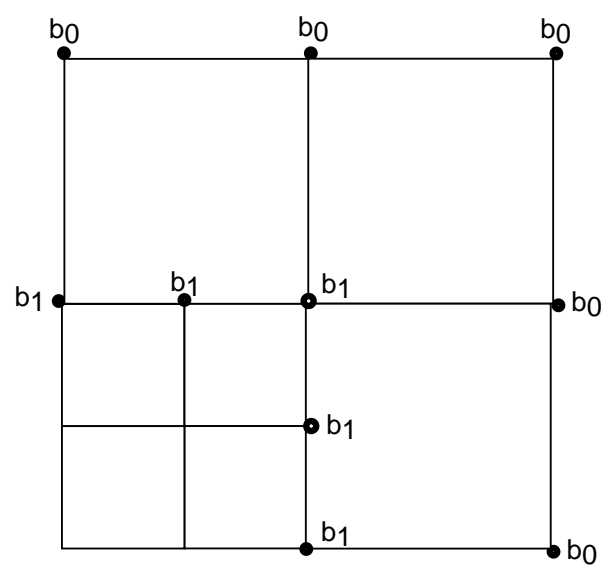

Figure 2.1. The first two steps in the construction

$\mathrm{PPH}_{m}$ is uniquely determined by its values on $V_{m}^{\prime} \times V_{m}^{\prime \prime}$ by a local version of (2.5) (it is easy to check that the extension is consistent on adjacent boundaries of cells, yielding a continuous function). The space $\mathrm{PPH}$ of all piecewise pluriharmonic functions is defined to be the union of $\mathrm{PPH}_{m}$ over all $m$.

Later we will show that $\mathrm{PPH}$ is dense in $\operatorname{dom} \mathcal{E}$. Now we will use a slight generalization of the $\mathrm{PPH}$ construction to obtain functions in $\operatorname{dom} \mathcal{E}$ that have point singularities. It is well known that in the case of the square there exist discontinuous functions in $\operatorname{dom} \mathcal{E}$, and in fact one can write down explicit examples, such as $\log \left(\left|\log \left(x^{2}+y^{2}\right)\right|\right)$. Such functions have rather mild singularities, since the Sobolev embedding theorem implies dom $\mathcal{E} \subseteq L^{q}$ for all $q<\infty$. The above example does not make use of the product structure of the square, so we offer another example which is a bit more awkward, but has the advantage that we can easily generalize it to other product spaces. The function we construct will have a singularity at the origin and be PPH with respect to an infinite set of subsquares obtained as follows: subdivide the square into 4 subsquares of level 1 and choose the 3 that do not contain the origin. For the remaining level 1 square, subdivide it into 4 subsquares of level 2 and choose the 3 that do not contain the origin. Iterate this procedure infinitely often. Given any sequence $\left\{b_{0}, b_{1}, \ldots\right\}$ construct the function $u$ by assigning the value $b_{0}$ to the 5 vertices $(1,0),\left(1, \frac{1}{2}\right),(1,1),\left(\frac{1}{2}, 1\right),(0,1)$ of the level 1 subsquares are not contained in the subsquare of level 1 containing the origin. The value $b_{1}$ is assigned to the 5 vertices $\left(\frac{1}{2}, 0\right),\left(\frac{1}{2}, \frac{1}{4}\right),\left(\frac{1}{2}, \frac{1}{2}\right),\left(\frac{1}{4}, \frac{1}{2}\right)$, $\left(0, \frac{1}{2}\right)$ of the level 2 subsquares that are not contained in the subsquare of level 2 containing the origin. And so on. See Figure 2.1. A simple computation shows that the contribution to the energy from the 3 level 1 squares in $\frac{8}{3}\left(b_{1}-b_{0}\right)^{2}$, the contribution from the 3 level 2 squares is $\frac{8}{3}\left(b_{2}-b_{1}\right)^{2}$, and so on. Thus the total energy is

$$
\frac{8}{3} \sum_{j=1}^{\infty}\left(b_{j}-b_{j-1}\right)^{2}
$$

We can make this finite while letting $b_{j} \rightarrow \infty$ by taking $b_{j}=\log j$. 
Theorem 2.4. Suppose the hypotheses of Lemma 2.2 hold with $b r_{j}^{\prime} r_{k}^{\prime \prime}>1$ for some $(j, k)$. Then there exists a function $u \in \operatorname{dom} \mathcal{E}$ with pole at $\left(q_{j}, q_{k}\right)$ that does not belong to $L^{q}$ for

$$
q>2+\frac{2 \log b}{\log \left(b r_{j}^{\prime} r_{k}^{\prime \prime}\right)}
$$

Proof. Given $q$ satisfying (2.6) choose the sequence $\left\{b_{0}, b_{1}, \ldots\right\}$ to be

$$
b_{m}=\left(b^{2} r_{j}^{\prime} r_{k}^{\prime \prime}\right)^{m / q} .
$$

Assign values to $u$ as follows: $u\left(q_{\ell}^{\prime}, q_{n}^{\prime \prime}\right)=b_{0}$ provided $(\ell, n) \neq(j, k) ; u\left(F_{j}^{\prime} q_{\ell}^{\prime}, F_{k}^{\prime \prime} q_{n}^{\prime \prime}\right)=$ $b_{1}$ provided $(\ell, n) \neq(j, k)$; and $u=b_{0}$ at all other points of $V_{1}^{\prime} \times V_{1}^{\prime \prime}$ except $\left(q_{j}^{\prime}, q_{k}^{\prime \prime}\right)$. Then extend $u$ to be PPH on all level 1 cells except $F_{j}^{\prime} K^{\prime} \times F_{k}^{\prime \prime} K^{\prime \prime}$. Next we assign values for $u$ at each of the vertices of order 2 lying in $F_{j}^{\prime} K^{\prime} \times F_{k}^{\prime \prime} K^{\prime \prime}$ except $\left(q_{j}, q_{k}\right)$, namely $u\left(\left(F_{j}^{\prime}\right)^{2} q_{\ell}^{\prime},\left(F_{k}^{\prime \prime}\right)^{2} q_{n}^{\prime \prime}\right)=b_{2}$ provided $(\ell, n) \neq(j, k)$, and $u=b_{1}$ at the remaining vertices. We extend $u$ to be PPH on all level 2 cells contained in $F_{j}^{\prime} K^{\prime} \times F_{k}^{\prime \prime} K^{\prime \prime}$ except $\left(F_{j}^{\prime}\right)^{2} K^{\prime} \times\left(F_{k}^{\prime \prime}\right)^{2} K^{\prime \prime}$. And so on.

The values of $u$ on the $V_{1}^{\prime} \times V_{1}^{\prime \prime}$ vertices of the level 1 cells (except $F_{j}^{\prime} K^{\prime} \times F_{k}^{\prime \prime} K^{\prime \prime}$ ) are either $b_{0}$ or $b_{1}$, so the contribution to the energy from these cells is exactly $c \frac{\left(b_{1}-b_{0}\right)^{2}}{\left(b r_{j}^{\prime} r_{k}^{\prime \prime}\right)}$ for a positive constant $c$ whose exact value is not important. But then the contributions to the energy from the level 2 cells is exactly $c \frac{\left(b_{2}-b_{1}\right)^{2}}{\left(b r_{j}^{\prime} r_{k}^{\prime \prime}\right)^{2}}$, and so on, so

$$
\mathcal{E}(u, u)=c \sum_{m=1}^{\infty} \frac{\left(b_{m}-b_{m-1}\right)^{2}}{\left(b r_{j}^{\prime} r_{k}^{\prime \prime}\right)^{m}} .
$$

We note that the choice $(2.7)$ allows us to bound $\mathcal{E}(u, u)$ by a convergent geometric sum exactly when (2.6) holds. On the other hand, similar estimates allow us to approximate $\|u\|_{q}^{q}$ from above and below by multiples of

$$
\sum_{m=1}^{\infty} b_{m}^{q}\left(\mu_{j}^{\prime} \mu_{k}^{\prime \prime}\right)^{m}=\sum_{m=1}^{\infty} \frac{b_{m}^{q}}{\left(b^{2} r_{j}^{\prime} r_{k}^{\prime \prime}\right)^{m}} .
$$

The choice (2.7) makes each term in (2.9) equal to 1 , so $u \notin L^{q}$.

We will see later that for many examples (2.6) is sharp, since dom $\mathcal{E} \subseteq L^{q}$ for $q$ equal to the right side of (2.6).

Because dom $\mathcal{E}$ contains discontinuous functions, we have to exercise some caution in working with pointwise values of functions in dom $\mathcal{E}$. However, it is clear from the definition that for $u \in \operatorname{dom} \mathcal{E}$ we must have $u\left(\cdot, x^{\prime \prime}\right) \in \operatorname{dom} \mathcal{E}^{\prime}$ and hence continuous for almost every choice of $x^{\prime \prime}$, and similarly $u\left(x^{\prime}, \cdot\right)$ is continuous for almost every $x^{\prime}$. So there is no harm in making this assumption (rather than just $u \in L^{2}$ ) from the beginning, which we will call minimal regularity.

Theorem 2.5. A function $u$ with minimal regularity belongs to dom $\mathcal{E}$ if and only if

$$
\sup _{m} \int_{K^{\prime \prime}} \mathcal{E}_{m}^{\prime}\left(u\left(\cdot, x^{\prime \prime}\right), u\left(\cdot, x^{\prime \prime}\right)\right) d \mu^{\prime \prime}\left(x^{\prime \prime}\right)
$$

and

$$
\sup _{m} \int_{K^{\prime}} \mathcal{E}_{m}^{\prime \prime}\left(u\left(x^{\prime}, \cdot\right), u\left(x^{\prime}, \cdot\right)\right) d \mu^{\prime}\left(x^{\prime}\right)
$$


are both finite. If $u$ and $v$ are in dom $\mathcal{E}$, then

$$
\begin{aligned}
\mathcal{E}(u, v)=\lim _{m \rightarrow \infty}\left(\int_{K^{\prime \prime}}\right. & \mathcal{E}_{m}^{\prime}\left(u\left(\cdot, x^{\prime \prime}\right), v\left(\cdot, x^{\prime \prime}\right)\right) d \mu^{\prime \prime}\left(x^{\prime \prime}\right) \\
& \left.+\int_{K^{\prime}} \mathcal{E}_{m}^{\prime \prime}\left(u\left(x^{\prime}, \cdot\right), v\left(x^{\prime}, \cdot\right)\right) d \mu^{\prime}\left(x^{\prime}\right)\right) .
\end{aligned}
$$

Proof. The minimal regularity implies $\mathcal{E}_{m}^{\prime}\left(u\left(\cdot, x^{\prime \prime}\right), u\left(\cdot, x^{\prime}\right)\right)$ is well defined for almost every $x^{\prime \prime}$, and $\mathcal{E}_{m}^{\prime \prime}\left(u\left(x^{\prime}, \cdot\right), u\left(x^{\prime}, \cdot\right)\right)$ is well defined for almost every $x^{\prime}$. Since $\mathcal{E}_{m}^{\prime}$ and $\mathcal{E}_{m}^{\prime \prime}$ are monotone increasing in $m$, if $u \in \operatorname{dom} \mathcal{E}$, then

$$
\mathcal{E}_{m}^{\prime}\left(u\left(\cdot, x^{\prime \prime}\right), u\left(\cdot, x^{\prime \prime}\right)\right) \leq \mathcal{E}^{\prime}\left(u\left(\cdot, x^{\prime \prime}\right), u\left(\cdot, x^{\prime \prime}\right)\right)
$$

so

$$
\int_{K^{\prime \prime}} \mathcal{E}_{m}^{\prime}\left(u\left(\cdot, x^{\prime \prime}\right), u\left(\cdot, x^{\prime \prime}\right)\right) d \mu^{\prime \prime}\left(x^{\prime \prime}\right) \leq \int_{K^{\prime \prime}} \mathcal{E}^{\prime}\left(u\left(\cdot, x^{\prime \prime}\right), u\left(\cdot, x^{\prime \prime}\right)\right) d \mu^{\prime \prime}\left(x^{\prime \prime}\right) \leq \mathcal{E}(u, u) .
$$

This shows (2.10) is finite, and similarly (2.11) is finite. Conversely, if (2.10) and (2.11) are finite, then the monotone convergence theorem shows that $u \in \operatorname{dom} \mathcal{E}$, and (2.12) holds for $u=v$. The general case of (2.12) follows by polarization.

Remark. Under the assumptions of Lemma 2.2 we can interpret this characterization in a way that is analogous to the construction of energy in the p.c.f. case. Let

$$
E_{0}(u, u)=\int_{K^{\prime \prime}} \mathcal{E}_{0}^{\prime}\left(u\left(\cdot, x^{\prime \prime}\right), u\left(\cdot, x^{\prime \prime}\right)\right) d \mu^{\prime \prime}\left(x^{\prime \prime}\right)+\int_{K^{\prime}} \mathcal{E}_{0}^{\prime \prime}\left(u\left(x^{\prime}, \cdot\right), u\left(x^{\prime}, \cdot\right)\right) d \mu^{\prime}\left(x^{\prime}\right)
$$

(the sum of the $m=0$ terms in (2.10) and (2.11)). We interpret this as a crude approximation to the energy at level 0 . Note that it depends only on the boundary values of $u$. We then create a more refined level $m$ approximate energy by taking

$$
E_{m}(u, u)=\sum_{\left|w^{\prime}\right|=m} \sum_{\left|w^{\prime \prime}\right|=m}\left(b^{m} r_{w^{\prime}}^{\prime} r_{w^{\prime \prime}}^{\prime \prime}\right)^{-1} E_{0}\left(u \circ\left(F_{w^{\prime}}^{\prime} \times F_{w^{\prime \prime}}^{\prime \prime}\right), u \circ\left(F_{w^{\prime}}^{\prime} \times F_{w^{\prime \prime}}^{\prime \prime}\right)\right),
$$

the sum over all cells of level $m$ of the appropriately renormalized crude energy of the restriction of $u$ to that cell. The point is that this is just the sum of the individual terms in (2.10) and (2.11); the assumptions of Lemma 2.2 guarantee the same renormalization factor for both terms. So (2.12) (for $u=v$ ) says that $\mathcal{E}(u, u)=\lim _{m \rightarrow \infty} E_{m}(u, u)$, and the limit is monotone increasing.

However, there are some differences compared to the p.c.f. case. We cannot assert that $E_{0}(u, u)$ is equal to $\mathcal{E}(u, u)$ if $u$ is harmonic, so $E_{0}(u, u)$ is not the "natural choice" for crude energy. We will return to this issue in Section 8. We do note that $E_{0}(u, u)$ gives the correct energy for pluriharmonic functions, so the limit in (2.12) is attained for finite $m$ if $u$ is PPH.

Another difference is that the renormalization factors in (2.3) do not uniquely (up to a constant multiple) determine the energy, as is often the case for p.c.f. fractals $[\mathrm{M}], \underline{\mathrm{P}},[\mathrm{Sa},[\mathrm{SST}]$. Indeed, we could modify (2.1) by multiplying each integral by an independent positive constant, and (2.3) would still hold. All these energies are different, although they are equivalent (each is bounded by a multiple of any other). We must choose $E_{0}(u, u)$ carefully in order to obtain the exact value of the energy in the limit.

The next characterization of energy involves "multiple Fourier series"; specifically, expansions in products of Neumann eigenfunctions for the Laplacians $\Delta^{\prime}$ and 
$\Delta^{\prime \prime}$. Let $\left\{u_{j}^{\prime}\right\}$ and $\left\{u_{k}^{\prime \prime}\right\}$ denote orthonormal bases for $L^{2}\left(K^{\prime}, \mu^{\prime}\right)$ and $L^{2}\left(K^{\prime \prime}, \mu^{\prime \prime}\right)$, respectively, of such eigenfunctions, so

$$
\left\{\begin{array}{l}
-\Delta^{\prime} u_{j}^{\prime}=\lambda_{j}^{\prime} u_{j}^{\prime}, \partial_{n}^{\prime} u_{j}^{\prime}\left(q_{\ell}^{\prime}\right)=0 \\
-\Delta^{\prime \prime} u_{k}^{\prime \prime}=\lambda_{k}^{\prime \prime} u_{k}^{\prime \prime}, \quad \partial_{n}^{\prime \prime} u_{k}^{\prime \prime}\left(q_{n}^{\prime \prime}\right)=0
\end{array}\right.
$$

with

$$
\left\{\begin{array}{l}
0=\lambda_{0}^{\prime}<\lambda_{1}^{\prime} \leq \lambda_{2}^{\prime} \leq \cdots \\
0=\lambda_{0}^{\prime \prime}<\lambda_{1}^{\prime \prime} \leq \lambda_{2}^{\prime \prime} \leq \cdots
\end{array}\right.
$$

Then clearly $u_{j k}\left(x^{\prime}, x^{\prime \prime}\right)=u_{j}^{\prime}\left(x^{\prime}\right) u_{k}^{\prime \prime}\left(x^{\prime \prime}\right)$ gives an orthonormal basis for $L^{2}(K)$. We will write

$$
u=\sum_{j=0}^{\infty} \sum_{k=0}^{\infty} a_{j k}(u) u_{j k}
$$

with

$$
a_{j k}(u)=\int_{K} u \cdot u_{j k} d \mu
$$

for the expansion of $u \in L^{2}(K)$ in this basis, so

$$
\int_{K} u v d \mu=\sum_{j=0}^{\infty} \sum_{k=0}^{\infty} a_{j k}(u) a_{j k}(v) .
$$

Theorem 2.6. $u \in \operatorname{dom} \mathcal{E}$ if and only if

$$
\sum_{j=0}^{\infty} \sum_{k=0}^{\infty}\left(\lambda_{j}^{\prime}+\lambda_{k}^{\prime \prime}\right) a_{j k}(u)^{2}
$$

is finite, in which case (2.18) equals $\mathcal{E}(u, u)$. More generally

$$
\sum_{j=0}^{\infty} \sum_{k=0}^{\infty}\left(\lambda_{j}^{\prime}+\lambda_{k}^{\prime \prime}\right) a_{j k}(u) a_{j k}(v)=\mathcal{E}(u, v)
$$

for $u$ and $v$ in $\operatorname{dom} \mathcal{E}$.

Proof. We use the analogous characterization of $\operatorname{dom} \mathcal{E}^{\prime}$ and dom $\mathcal{E}^{\prime \prime}$; specifically $u^{\prime} \in \operatorname{dom} \mathcal{E}^{\prime}$ if and only if $\sum_{j=0}^{\infty} \lambda_{j}^{\prime} a_{j}^{\prime}\left(u^{\prime}\right)^{2}$ is finite, in which case it equals $\mathcal{E}^{\prime}\left(u^{\prime}, u^{\prime}\right)$, for $a_{j}^{\prime}\left(u^{\prime}\right)=\int_{K^{\prime}} u^{\prime} u_{j}^{\prime} d \mu^{\prime}$, and similarly for dom $\mathcal{E}^{\prime \prime}$. This result is well known but perhaps not stated explicitly in the literature. A proof for the special case SG is given in [S5], Theorem 3.7 (d), and the same proof works in general. Note that it is important to use Neumann eigenfunctions, as the analogous statement is not valid for Dirichlet eigenfunctions.

The proof is now easy. Fix $x^{\prime \prime}$ and note that

$$
\mathcal{E}^{\prime}\left(u\left(\cdot, x^{\prime \prime}\right), u\left(\cdot, x^{\prime \prime}\right)\right)=\sum_{j=0}^{\infty} \lambda_{j}^{\prime}\left(\sum_{k=0}^{\infty} a_{j k}(u) u_{k}^{\prime \prime}\left(x^{\prime \prime}\right)\right)^{2}
$$


because $\sum_{k=0}^{\infty} a_{j k}(u) u_{k}^{\prime \prime}\left(x^{\prime \prime}\right)=a_{j}^{\prime}\left(u\left(\cdot, x^{\prime \prime}\right)\right)$. Integrate with respect to $x^{\prime \prime}$ and use the orthonormality of $\left\{u_{k}^{\prime \prime}\right\}$ to obtain

$$
\int_{K^{\prime \prime}} \mathcal{E}^{\prime}\left(u\left(\cdot, x^{\prime \prime}\right), u\left(\cdot, x^{\prime \prime}\right)\right) d \mu^{\prime \prime}\left(x^{\prime \prime}\right)=\sum_{j=0}^{\infty} \lambda_{j}^{\prime}\left(\sum_{k=0}^{\infty} a_{j k}(u)^{2}\right) .
$$

Do a similar argument in the reverse order and add the two to obtain the equality of $(2.18)$ and $\mathcal{E}(u, u)$. The rest of the argument is routine.

Corollary 2.7. (a) For $u \in \operatorname{dom} \mathcal{E}, \mathcal{E}(u, u)=0$ if and only if $u$ is constant. (b) The space dom $\mathcal{E}$ modulo constants forms a Hilbert space with inner product $\mathcal{E}(u, v)$. (c) dom $\mathcal{E}$ is dense in $L^{2}$. (d) dom $\mathcal{E} /$ constants $\subseteq L^{2}$ is compact. (e) PPH is dense in $\operatorname{dom} \mathcal{E}$.

Proof. Note that $u_{0}^{\prime}, u_{0}^{\prime \prime}$ and $u_{00}$ are all constant, and $\lambda_{j}^{\prime}+\lambda_{k}^{\prime \prime}=0$ if and only if $j=k=0$. (a), (b) and (c) follow easily. (d) follows because the sequence $\left\{\lambda_{j}^{\prime}+\lambda_{k}^{\prime \prime}\right\}$ arranged in increasing order tends to infinity. To prove (e) note that finite sums of the form (2.15) are dense in $\operatorname{dom} \mathcal{E}$, so it suffices to show that each $u_{j k}$ may be approximated in energy by $\mathrm{PPH}$ functions. But $u_{j}^{\prime}$ and $u_{k}^{\prime \prime}$ may each be approximated, both in $L^{2}$ and energy, by piecewise harmonic functions $\varphi_{j \varepsilon}^{\prime}$ and $\varphi_{k \varepsilon}^{\prime \prime}$, so $\varphi_{j \varepsilon}^{\prime}\left(x^{\prime}\right) \varphi_{k \varepsilon}^{\prime \prime}\left(x^{\prime \prime}\right)$ approximates $u_{j k}$ in energy.

The next result says that $\mathcal{E}$ satisfies the Markov property.

Theorem 2.8. Let $u \in \operatorname{dom} \mathcal{E}$ and let $v(x)=\max (a, \min (u(x), b))$ for any fixed constants $a, b$ with $a<b$. Then $v \in \operatorname{dom} \mathcal{E}$ and $\mathcal{E}(v, v) \leq \mathcal{E}(u, u)$.

Proof. This result is known for $\mathcal{E}^{\prime}$ and $\mathcal{E}^{\prime \prime}$, and it transfers to $\mathcal{E}$ directly from $(2.1)$.

For the next energy formula, using the method of average values originally due to Kusuoka and Zhou [KZ], we restrict attention to $K=\mathrm{SG}^{2}$. No doubt there are analogous results in the general case, but the preliminary work in the p.c.f. case has not yet been done. For a function $u^{\prime}$ defined on $\mathrm{SG}$, we define the averages on cells $F_{w^{\prime}}^{\prime}(\mathrm{SG})$ by

$$
\begin{aligned}
A_{w^{\prime}}\left(u^{\prime}\right) & =3^{m} \int_{F_{w^{\prime}}^{\prime}(\mathrm{SG})} u^{\prime}\left(x^{\prime}\right) d \mu^{\prime}\left(x^{\prime}\right) \\
& =\int_{\mathrm{SG}} u^{\prime}\left(F_{w^{\prime}}^{\prime} x^{\prime}\right) d \mu^{\prime}\left(x^{\prime}\right)
\end{aligned}
$$

for $\left|w^{\prime}\right|=m$. Two cells of level $m$ are adjacent if they have a point in common, and we write $w^{\prime} \sim_{m} \widetilde{w}^{\prime}$. Note that a nonboundary cell always has exactly three neighbors. In $[\mathrm{S} 4]$ we showed that

$$
\mathcal{E}^{\prime}\left(u^{\prime}, u^{\prime}\right)=\lim _{m \rightarrow \infty} \frac{3}{2}\left(\frac{5}{3}\right)^{m} \sum_{w^{\prime} \sim_{m} \widetilde{w}^{\prime}}\left(A_{w^{\prime}}\left(u^{\prime}\right)-A_{\widetilde{w}^{\prime}}\left(u^{\prime}\right)\right)^{2},
$$

so dom $\mathcal{E}^{\prime}$ is characterized by the finiteness of the limit on the right of $(2.21)$. In fact we showed more precisely that

$$
\mathcal{E}_{m}^{\prime}\left(u^{\prime}, u^{\prime}\right)=\frac{3}{2}\left(\left(\frac{3}{5}\right)^{m}-\left(\frac{3}{5}\right)^{2 m}\right)^{-1} \sum_{w^{\prime} \sim_{m} \widetilde{w}^{\prime}}\left(A_{w^{\prime}}\left(u^{\prime}\right)-A_{\widetilde{w}^{\prime}}\left(u^{\prime}\right)\right)^{2}
$$


if $u^{\prime}$ is harmonic, so that by adjusting the constant in (2.21) we have a monotone limit.

Similarly, for $u$ defined on $\mathrm{SG}^{2}$ and a cell $F_{w}\left(\mathrm{SG}^{2}\right)$ of level $m$, so $w=\left(w^{\prime}, w^{\prime \prime}\right)$ with $\left|w^{\prime}\right|=\left|w^{\prime \prime}\right|=m$, we define the average

$$
\begin{aligned}
A_{w}(u) & =9^{m} \int_{F_{w^{\prime}}^{\prime}(\mathrm{SG})} \int_{F_{w^{\prime \prime}}^{\prime \prime}(\mathrm{SG})} u\left(x^{\prime}, x^{\prime \prime}\right) d \mu^{\prime}\left(x^{\prime}\right) d \mu^{\prime \prime}\left(x^{\prime \prime}\right) \\
& =\int_{\mathrm{SG}^{2}} u\left(F_{w} x\right) d \mu(x) .
\end{aligned}
$$

The adjacency relation $w \sim_{m} \widetilde{w}$ will mean that either $w^{\prime} \sim_{m} \widetilde{w}^{\prime}$ and $w^{\prime \prime}=\widetilde{w}^{\prime \prime}$, or $w^{\prime}=\widetilde{w}^{\prime}$ and $w^{\prime \prime} \sim_{m} \widetilde{w}^{\prime \prime}$. In other words, the two cells must intersect along a set equivalent to $\mathrm{SG}$, not just at an isolated point.

Theorem 2.9. For $u$ and $v$ in $\operatorname{dom} \mathcal{E}$ on $S G^{2}$,

$$
\mathcal{E}(u, v)=\lim _{m \rightarrow \infty} \frac{3}{2}\left(\frac{5}{9}\right)^{m} \sum_{w \sim_{m} \widetilde{w}}\left(A_{w}(u)-A_{\widetilde{w}}(u)\right)\left(A_{w}(v)-A_{\widetilde{w}}(v)\right) .
$$

Proof. Suppose $w^{\prime} \sim_{m} \widetilde{w}^{\prime}$ and $w^{\prime \prime}=\widetilde{w}^{\prime \prime}$. Then

$$
A_{w}(u)-A_{\widetilde{w}}(u)=3^{m} \int_{F_{w^{\prime \prime}}^{\prime \prime}(\mathrm{SG})}\left(A_{w^{\prime}}\left(u\left(\cdot, x^{\prime \prime}\right)\right)-A_{\widetilde{w}^{\prime}}\left(u\left(\cdot, x^{\prime \prime}\right)\right)\right) d \mu^{\prime \prime}\left(x^{\prime \prime}\right) .
$$

By Cauchy-Schwartz

$$
\begin{aligned}
& \sum_{\left|w^{\prime \prime}\right|=m} \sum_{w^{\prime} \sim_{m} \widetilde{w}^{\prime}}\left|A_{w}(u)-A_{\widetilde{w}}(u)\right|^{2} \\
& \quad \leq 3^{m} \int_{\mathrm{SG}} \sum_{w^{\prime} \sim_{m} \widetilde{w}^{\prime}}\left|A_{w^{\prime}}\left(u\left(\cdot, x^{\prime \prime}\right)\right)-A_{\widetilde{w}^{\prime}}\left(u\left(\cdot, x^{\prime \prime}\right)\right)\right|^{2} d \mu\left(x^{\prime \prime}\right)
\end{aligned}
$$

hence

$$
\begin{gathered}
\lim _{m \rightarrow \infty}\left(\frac{3}{2}\right)\left(\frac{5}{9}\right)^{m} \sum_{\left|w^{\prime \prime}\right|=m} \sum_{w^{\prime} \sim_{m} \widetilde{w}^{\prime}}\left|A_{w}(u)-A_{\widetilde{w}}(u)\right|^{2} \\
\leq \int_{\mathrm{SG}} \mathcal{E}^{\prime}\left(u\left(\cdot, x^{\prime \prime}\right), u\left(\cdot, x^{\prime \prime}\right)\right) d \mu^{\prime \prime}\left(x^{\prime \prime}\right)
\end{gathered}
$$

by (2.21) and the monotone convergence theorem. By a similar argument in the case $w^{\prime}=\widetilde{w}^{\prime}$ and $w^{\prime \prime} \sim_{m} \widetilde{w}^{\prime \prime}$, and adding, we see that

$$
\lim _{m \rightarrow \infty} \frac{3}{2}\left(\frac{5}{9}\right)^{m} \sum_{w \sim_{m} \widetilde{w}}\left(A_{w}(u)-A_{\widetilde{w}}(u)\right)^{2} \leq \mathcal{E}(u, u) .
$$

If $u$ is also continuous, it is easy to see that we have equality in (2.25). In particular, if $u$ is represented by a finite sum in (2.15). Such functions are dense in $\operatorname{dom} \mathcal{E}$, and we may use $(2.25)$ to control the remainder, to obtain equality for all $u \in \operatorname{dom} \mathcal{E}$. By polarization we then obtain (2.24).

It seems likely that there is a full equivalence, so that the finiteness of the limit in $(2.25)$ for $u$ of minimal regularity would imply $u \in \operatorname{dom} \mathcal{E}$. However, the proof would have to be quite technical, so we will not attempt it here. 


\section{HARMONIC FUNCTIONS}

In this section we require the assumptions of Lemma 2.2. We want to define harmonic functions as energy minimizers, subject to boundary conditions. We observe that functions in dom $\mathcal{E}$ have well-defined boundary values in $L^{2}$ by Theorem 2.5. The converse is of course not true. In Section 7 we will be able to give a rather complete description of the "trace space" of $\operatorname{dom} \mathcal{E}$, but we will side-step the issue in this section by simply assuming that we are given a function $\varphi$ in $L^{2}(\partial K)$ that is the boundary value of a function in $\operatorname{dom} \mathcal{E}$.

Theorem 3.1. Let $\varphi \in L^{2}(\partial K)$ be the boundary values of some $u \in \operatorname{dom} \mathcal{E}$. Then there is a unique function $h \in$ dom $\mathcal{E}$ which achieves the minimum value of $\mathcal{E}(v, v)$ for all $v \in \operatorname{dom} \mathcal{E}$ with $\left.v\right|_{\partial K}=\varphi$. Moreover $h$ depends linearly on $\varphi$.

Proof. Let $B_{\varphi}$ be the subset of dom $\mathcal{E}$ given by boundary condition $\left.v\right|_{\partial K}=\varphi \cdot B_{\varphi}$ is an affine subspace transverse to the constants, so $\mathcal{E}(u-v, u-v)^{1 / 2}$ is a metric on $B_{\varphi}$, and $B_{\varphi}$ is closed and hence complete in this metric (using Theorem 2.5 and Corollary $2.7(\mathrm{~b}))$. Let $m$ denote the infinum of $\mathcal{E}(u, u)$ over $B_{\varphi}$. The key estimate is

$$
\frac{1}{2}(\mathcal{E}(u, u)+\mathcal{E}(v, v))-\mathcal{E}\left(\frac{u-v}{2}, \frac{u-v}{2}\right)=\mathcal{E}\left(\frac{u+v}{2}, \frac{u+v}{2}\right) \geq m
$$

for $u$ and $v$ in $B_{\varphi}$, since $B_{\varphi}$ is convex. If $\left\{u_{k}\right\}$ is a minimizing sequence in $B_{\varphi}$, then (3.1) implies

$$
\mathcal{E}\left(u_{j}-u_{k}, u_{j}-u_{k}\right) \leq 2\left(\mathcal{E}\left(u_{j}, u_{j}\right)+\mathcal{E}\left(u_{k}, u_{k}\right)\right)-4 m,
$$

which shows that $\left\{u_{k}\right\}$ is a Cauchy sequence. By completeness, $u_{k}$ converges in the energy metric. Call the limit $h$. Clearly $h$ is a minimizer. The uniqueness also follows by applying (3.1) to two minimizers $h_{1}$ and $h_{2}$. We obtain

$$
\mathcal{E}\left(h_{1}-h_{2}, h_{1}-h_{2}\right)=0
$$

so $h_{1}=h_{2}$ because they are equal on the boundary.

Definition 3.2. A function $h$ in $\operatorname{dom} \mathcal{E}$ is said to be a harmonic function of finite energy if $h$ minimizes energy among functions with the same boundary values. If $\Omega$ is any open set in $K$ and $h$ is a function defined on $\Omega$, we say $h$ is harmonic in $\Omega$ if for any simple subset $C=\bigcup F_{w} K$ contained in the interior of $\Omega, h$ minimizes $\mathcal{E}_{C}(u, u)$ over all functions $u$ with the same values on $\partial C$.

In order for the definition to be consistent we need to verify the following:

Lemma 3.3. If $h$ is harmonic with finite energy, then $h \circ F_{w}$ is harmonic with finite energy for any word $w$.

Proof. By (2.3) iterated we may write the energy of $h$ as a sum of contributions from all cells of a fixed level $m$,

$$
\mathcal{E}(h, h)=\sum_{|w|=m}\left(b^{m} r_{w}\right)^{-1} \mathcal{E}\left(h \circ F_{w}, h \circ F_{w}\right) .
$$

Suppose for one choice $\widetilde{w}$ of $w$ the function $h \circ F_{\widetilde{w}}$ did not achieve the minimum for its boundary values, so $\mathcal{E}(v, v)<\mathcal{E}\left(h \circ F_{\widetilde{w}}, h \circ F_{\widetilde{w}}\right)$ while $\left.v\right|_{\partial K}=\left.h \circ F_{\widetilde{w}}\right|_{\partial K}$. Then 
we could construct a function $\widetilde{h}$ as follows:

$$
\widetilde{h}(x)= \begin{cases}h(x) & \text { if } x \notin F_{\widetilde{w}} K, \\ v\left(F_{\widetilde{w}}^{-1} x\right) & \text { if } x \in F_{\widetilde{w}} K .\end{cases}
$$

The boundary conditions imply that $\widetilde{h}=h$ on the closure of the complement of $F_{\widetilde{w}} K$, so when we compute $\mathcal{E}(\widetilde{h}, \widetilde{h})$ using $(3.2)$ all the terms corresponding to $w \neq \widetilde{w}$ are the same, while the one corresponding to $w=\widetilde{w}$ is smaller. But $\left.\widetilde{h}\right|_{\partial K}=\left.h\right|_{\partial K}$, contradicting the minimality of $h$.

Harmonic functions of finite energy satisfy a form of the maximum principle.

Theorem 3.4. Let $h$ be a harmonic function of finite energy with boundary values $\varphi$. Suppose $\varphi$ is bounded from above (or below). Then $h$ is bounded from above (or below) by the same bounds.

Proof. Suppose $\varphi \leq M$. Let $v(x)=\min (u(x), M)$. Then $v$ has the same boundary values as $h$. But $\mathcal{E}(v, v) \leq \mathcal{E}(h, h)$ by Theorem 2.8 , so by the uniqueness of the minimizer in Theorem 3.1 we must have $v=h$, hence $h \leq M$. The same argument works for lower bounds.

The same result localizes to any simple subset.

It follows by the Hahn-Banach theorem that we have a "harmonic measure" representation of $h$ in terms of its boundary values: for each $x$ in the interior of $K$ there exists a probability measure $\nu_{x}$ on $\partial K$ such that

$$
h(x)=\int_{\partial K} \varphi(y) d \nu_{x}(y) .
$$

In Section 8 we will give a more precise description of the harmonic measure as a "Poisson integral".

\section{The Laplacian}

Given the energy constructed in Section 2 and a reasonable measure $\nu$ on $K$, we may define a Laplacian $\Delta_{\nu}$ via the weak formulation

$$
-\mathcal{E}(u, v)=\int_{K}\left(\Delta_{\nu} u\right) v d \nu
$$

for all $v \in \operatorname{dom}_{0} \mathcal{E}$, the subspace of dom $\mathcal{E}$ of functions vanishing on the boundary. For simplicity we will restrict attention to the case $\nu=\mu$.

Definition 4.1. For $u \in \operatorname{dom} \mathcal{E}$ and $f \in C(K)$, we say $u \in \operatorname{dom} \Delta$ and $\Delta u=f$ provided

$$
-\mathcal{E}(u, v)=\int f v d \mu \text { for all } v \in \operatorname{dom}_{0} \mathcal{E} .
$$

If $f \in L^{q}(K)$ for some $q \geq 2$ and (4.2) holds, we say $u \in \operatorname{dom}_{L^{q}} \Delta$. Note that the right side of (4.2) is well defined since $\operatorname{dom} \mathcal{E} \subseteq L^{2}$. (Later we will be able to reduce the lower bound on $q$, but $q=2$ is the case we will consider most often.) Note that it suffices to verify (4.2) for all $v \in \mathrm{PPH}$ vanishing on the boundary, by Corollary 2.7 (c). If $\Omega$ is an open set in the interior of $K$, we will say $\Delta u=f$ on $\Omega$ if (4.2) holds for all $v \in \operatorname{dom} \mathcal{E}$ with support in $\Omega$. Here we may allow $f \in C(\Omega)$ or $f \in L_{\mathrm{loc}}^{q}(\Omega)$.

It is easy to see that $\operatorname{dom}_{0} \mathcal{E}$ is dense in $L^{2}$, so $f$ is uniquely determined by (4.2). 
Next we give a variational formulation of $\Delta u=f$.

Theorem 4.2. Let $u \in$ dom $\mathcal{E}$ with boundary values $\varphi$, and let $B_{\varphi}$ denote the affine subspace of dom $\mathcal{E}$ of all functions with boundary values $\varphi$. Let $f \in C(K)$ (respectively $f \in L^{q}(K)$ for some $q \geq 2$ ). Then $u \in$ dom $\Delta$ (respectively $u \in$ $\left.\operatorname{dom}_{L^{q}} \Delta\right)$ and $\Delta u=f$ if and only if $u$ minimizes

$$
\frac{1}{2} \mathcal{E}(v, v)+\int f v d \mu
$$

over all $v \in B_{\varphi}$. Moreover, the minimum is unique.

Proof. Note that (4.3) is a strictly convex functional on $B_{\varphi}$, so every critical point is a minimum, and there is at most one minimum. So it suffices to show that critical points of (4.3) are the same as solutions of (4.2). Also note that if $v \in \operatorname{dom}_{0} \mathcal{E}$, then $(u+t v) \in B_{\varphi}$ for every real $t$. Thus we may substitute $u+t v$ in (4.3). The result is

$$
\frac{1}{2} \mathcal{E}(u, u)+t \mathcal{E}(u, v)+\frac{1}{2} t^{2} \mathcal{E}(v, v)+\int f u d \mu+t \int f v d \mu .
$$

Clearly the derivative vanishes at $t=0$ if and only if (4.2) holds.

Corollary 4.3. A function $u$ is harmonic with finite energy if and only if $u \in$ dom $\Delta$ and $\Delta u=0$. If $\Omega$ is any open set in the interior of $K$, then $u$ is harmonic on $\Omega$ if and only if $\Delta u=0$ on $\Omega$.

Proof. The first statement is an immediate consequence of Theorem 4.2 and Definition 3.2. Next suppose $\Delta u=0$ on $\Omega$, and let $C$ denote any simple subset with $C \subseteq \Omega$. Then any function $v$ vanishing on the boundary of $C$ may be extended to zero outside $C$, with $\mathcal{E}(u, v)=\mathcal{E}_{C}(u, v)$, and $v \in \operatorname{dom} \mathcal{E}_{C}$ if and only if $v \in \operatorname{dom} \mathcal{E}$. We may then repeat the argument in the proof of Theorem 4.2 to conclude that $u$ minimizes the energy in $C$ among functions with the same boundary values on $\partial C$. Thus $u$ is harmonic. Conversely, assume $u$ is harmonic and let $v \in \operatorname{dom} \mathcal{E}$ with supp $v \subseteq \Omega$. Since supp $v$ is compact, we can find a simple subset $C$ such that supp $v \subseteq C \subseteq \Omega$. Since $u$ minimizes energy in $C$ among functions with the same boundary values on $\partial C$, the above generalization of the proof of Theorem 4.2 shows that $\mathcal{E}(u, v)=0$. Thus $\Delta u=0$ on $\Omega$.

Theorem 4.4. Given any $f \in C(K)$ (respectively $f \in L^{q}(K)$ for some $q \geq 2$ ), there exists a unique solution to the Dirichlet problem $u \in$ dom $\Delta$ (respectively $u \in \operatorname{dom}_{L^{2}} \Delta$ ) with

$$
\Delta u=f,\left.\quad u\right|_{\partial K}=0 .
$$

Proof. We need to find a minimizer of (4.3) in $\operatorname{dom}_{0} \mathcal{E}$. First we claim that (4.3) is bounded from below on $\operatorname{dom}_{0} \mathcal{E}$. This follows easily from

$$
\|v\|_{2}^{2} \leq c \mathcal{E}(v, v) \quad \text { for } \quad v \in \operatorname{dom}_{0} \mathcal{E}
$$

a consequence of Corollary 2.7 (d) and the fact that $\operatorname{dom}_{0} \mathcal{E}$ is a closed subspace of $\operatorname{dom} \mathcal{E}$ containing no nonzero constants. Let $m$ denote the infimum of (4.3) over $\operatorname{dom}_{0} \mathcal{E}$, and let $\left\{v_{k}\right\}$ be a minimizing sequence. The argument is then much the 
same as in the proof of Theorem 3.1, the only difference is that we replace (3.1) by

$$
\begin{aligned}
\frac{1}{2}\left(\frac{1}{2} \mathcal{E}\left(v_{j}, v_{j}\right)+\int f v_{j} d \mu+\frac{1}{2} \mathcal{E}\left(v_{k}, v_{k}\right)+\int f v_{k} d \mu\right) \\
-\frac{1}{2} \mathcal{E}\left(\frac{v_{j}-v_{k}}{2}, \frac{v_{j}-v_{k}}{2}\right) \\
=\frac{1}{2} \mathcal{E}\left(\frac{v_{j}+v_{k}}{2}, \frac{v_{j}+v_{k}}{2}\right)+\int f\left(\frac{v_{j}+v_{k}}{2}\right) d \mu \geq m .
\end{aligned}
$$

We next consider a method to compute $\Delta u$ in some, but by no means all cases, based on the intuition that $\Delta=\Delta^{\prime}+\Delta^{\prime \prime}$.

Theorem 4.5. Suppose we are given continuous functions $u, f_{1}, f_{2}$, such that for all $x^{\prime \prime}, u\left(\cdot, x^{\prime \prime}\right) \in$ dom $\Delta^{\prime}$ and $\Delta^{\prime} u\left(\cdot, x^{\prime \prime}\right)=f_{1}\left(\cdot, x^{\prime \prime}\right)$, while also for all $x^{\prime}$, $u\left(x^{\prime}, \cdot\right) \in \operatorname{dom} \Delta^{\prime \prime}$ and $\Delta^{\prime \prime} u\left(x^{\prime}, \cdot\right)=f_{2}\left(x^{\prime}, \cdot\right)$. Then $u \in$ dom $\Delta$ and $\Delta u=f_{1}+f_{2}$. In particular, if $u\left(x^{\prime}, x^{\prime \prime}\right)=u^{\prime}\left(x^{\prime}\right) u^{\prime \prime}\left(x^{\prime \prime}\right)$ for $u^{\prime} \in$ dom $\Delta^{\prime}$ and $u^{\prime \prime} \in$ dom $\Delta^{\prime \prime}$, then

$$
\Delta u\left(x^{\prime}, x^{\prime \prime}\right)=\Delta^{\prime} u^{\prime}\left(x^{\prime}\right) u^{\prime \prime}\left(x^{\prime \prime}\right)+u^{\prime}\left(x^{\prime}\right) \Delta^{\prime \prime} u^{\prime \prime}\left(x^{\prime \prime}\right) .
$$

Proof. For $v \in \operatorname{dom}_{0} \mathcal{E}$, for almost every $x^{\prime \prime}, v\left(\cdot, x^{\prime \prime}\right) \in \operatorname{dom}_{0} \mathcal{E}^{\prime}$, so

$$
-\mathcal{E}^{\prime}\left(u\left(\cdot, x^{\prime \prime}\right), v\left(\cdot, x^{\prime \prime}\right)\right)=\int f_{1}\left(x^{\prime}, x^{\prime \prime}\right) v\left(x^{\prime}, x^{\prime \prime}\right) d \mu^{\prime}\left(x^{\prime}\right)
$$

by the definition of $\Delta^{\prime}$. Since $f, v \in L^{2} \subseteq L^{1}$ we may integrate (4.8) to obtain

$$
-\int \mathcal{E}^{\prime}\left(u\left(\cdot, x^{\prime \prime}\right), v\left(\cdot, x^{\prime \prime}\right)\right) d \mu^{\prime \prime}\left(x^{\prime \prime}\right)=\int f_{1} v d \mu .
$$

Running the argument in the other order and adding the results yields

$$
-\mathcal{E}(u, v)=\int\left(f_{1}+f_{2}\right) v d \mu
$$

We easily observe that $\Delta$ satisfies the scaling identity

$$
\Delta\left(u \circ F_{w}\right)=b^{-m}(\Delta u) \circ F_{w} \text { for }|w|=m
$$

under the hypotheses of Lemma 2.2.

Next we prove a removable singularities type theorem.

Theorem 4.6. Assume the hypotheses of Lemma 2.2 with $b r_{i}^{\prime} r_{j}^{\prime \prime}>1$. Suppose $u \in \operatorname{dom} \mathcal{E}$ satisfies $\Delta u=f$ on $K \backslash\{z\}$ for $f \in L^{2}$, where $z$ is any fixed point in the interior of $K$. Then $u \in d_{0 m_{L^{2}}} \Delta$ and satisfies $\Delta u=f$ on $K$.

Proof. We need to show that (4.2) holds for all $v \in \mathrm{PPH}$ vanishing on the boundary, and by hypothesis we know it holds if $v$ also vanishes in a neighborhood of $z$. So we approximate $v \in \mathrm{PPH}$ by a sequence $v_{m}$ where $v_{m}$ vanishes near $z$. This is easily done: just take the data $\left.v\right|_{V_{m}}$ and change all the values $v(x)$ to 0 for $x$ in any $m$-cell containing $z$. Then $v_{m} \in \mathrm{PPH}_{m}$ is determined by the new data. We have

$$
-\mathcal{E}\left(u, v_{m}\right)=\int f v_{m} d \mu .
$$


It is easy to see that the right side of (4.10) tends to $\int f v d \mu$ as $m \rightarrow \infty$, so it remains to show that the left side tends to $-\mathcal{E}(u, v)$. For this we use the estimate

$$
\left|\mathcal{E}\left(u, v-v_{m}\right)\right| \leq \mathcal{E}(u, u)^{1 / 2} \mathcal{E}\left(v-v_{m}, v-v_{m}\right)^{1 / 2} .
$$

Finally, it is easy to see that $\mathcal{E}\left(v-v_{m}, v-v_{m}\right) \rightarrow 0$ because $b r_{i}^{\prime} r_{j}^{\prime \prime}>1$.

This is not the optimal result. In the case of the square, $b r_{i}^{\prime} r_{j}^{\prime \prime}=1$. The result is still true, but it requires a more delicate approximation argument. In the case $b r_{i}^{\prime} r_{j}^{\prime \prime}>1$, the result should be true under weaker hypotheses on $u$. It should not be necessary to assume $u \in \operatorname{dom} \mathcal{E}$ on all of $K$, but only on $K \backslash \Omega$ for any neighborhood $\Omega$ of $z$, and in addition $u \in L^{q}(K)$ for the appropriate choice of $q$ (see Corollary $6.2(\mathrm{c}))$.

\section{The QUADRUPLE COVER}

Let $\widetilde{K}^{\prime}$ denote the double cover of $K^{\prime}$, so $\widetilde{K}^{\prime}$ consists of two copies of $K^{\prime}$ identified at all boundary points. In many cases, including SG, $\widetilde{K}^{\prime}$ fits into the fractafold framework described in [S6], in that a neighborhood of an identified boundary point is isomorphic to a neighborhood in $K^{\prime}$. But in any case, it is easy to extend the definition of $\mathcal{E}^{\prime}$ and $\operatorname{dom} \mathcal{E}^{\prime}$ to $\widetilde{K}^{\prime}$, and then $\Delta^{\prime}$, dom $\Delta^{\prime}$ and $\operatorname{dom}_{L^{2}} \Delta^{\prime}$ via the weak formulation (in the definition of $\Delta^{\prime} u$ we allow the test function $v$ to vary over all of dom $\widetilde{\mathcal{E}}^{\prime}$, since $\widetilde{K}^{\prime}$ has no boundary). We denote all these extensions by placing a tilde over the corresponding symbol. Similarly, let $\widetilde{K}^{\prime \prime}$ denote the double cover of $K^{\prime \prime}$. The quadruple cover $\widetilde{K}$ of $K$ is then defined to be $\widetilde{K}=\widetilde{K}^{\prime} \times \widetilde{K}^{\prime \prime}$. In the case $K^{\prime}=K^{\prime \prime}=S G$ it is easy to verify that every point in $\widetilde{K}$ has a neighborhood isomorphic to a neighborhood of a point in $K$, so the fractafold concept is also relevant. We may easily extend the definitions of energy and Laplacian to $\widetilde{K}$. Most of the results proved previously for $K$ extend to $\widetilde{K}$ with obvious modifications. The main simplification, due to the lack of boundary for $\widetilde{K}$, is that we can extend the spectral description of dom $\widetilde{\mathcal{E}}$ to $\operatorname{dom}_{L^{2}} \widetilde{\Delta}$.

To begin, we observe that the spectrum of $\widetilde{\Delta}^{\prime}$ on $\widetilde{K}^{\prime}$ is just the union of the Dirichlet and Neumann spectra of $\Delta^{\prime}$ on $K^{\prime}$. If $\left\{u_{j, D}^{\prime}\right\}$ and $\left\{u_{j, N}^{\prime}\right\}$ denote orthonormal bases of Dirichlet and Neumann eigenfunctions of $\Delta^{\prime}$ on $K^{\prime}$, then we essentially take odd and even reflections (except for a normalization factor) to extend these functions to $\widetilde{K}^{\prime}$ to be eigenfunctions of $\widetilde{\Delta}^{\prime}$. In this way we obtain an orthonormal basis of $L^{2}\left(\widetilde{K}^{\prime}\right)$, which we denote simply as $\left\{\widetilde{u}_{j}^{\prime}\right\}$, and the corresponding eigenvalues $\left\{\widetilde{\lambda}_{j}^{\prime}\right\}$, which we assume are arranged in nondecreasing order, with $\widetilde{\lambda}_{0}^{\prime}=0$ corresponding to the constant function, and all other $\widetilde{\lambda}_{j}^{\prime}$ strictly positive. If we denote the expansion of an $L^{2}$ function as

$$
u^{\prime}=\sum_{j=0}^{\infty} a_{j}\left(u^{\prime}\right) \widetilde{u}_{j}^{\prime}
$$

then $\operatorname{dom} \widetilde{\mathcal{E}}^{\prime}$ is characterized by

$$
\sum_{j=1}^{\infty} \widetilde{\lambda}_{j}^{\prime} a_{j}\left(u^{\prime}\right)^{2}=\widetilde{\mathcal{E}}\left(u^{\prime}, u^{\prime}\right)<\infty
$$


and $\operatorname{dom}_{L^{2}} \widetilde{\Delta}^{\prime}$ is characterized by

$$
\sum_{j=1}^{\infty}\left(\widetilde{\lambda}_{j}^{\prime}\right)^{2} a_{j}\left(u^{\prime}\right)^{2}=\left\|\widetilde{\Delta}^{\prime} u^{\prime}\right\|_{2}^{2}<\infty .
$$

These observations are rather straightforward, and also may be gleaned from Ki3.

Next we observe that by taking the tensor product of the bases $\left\{\widetilde{u}_{j}^{\prime}\right\}$ and $\left\{\widetilde{u}_{j}^{\prime \prime}\right\}$ we obtain an orthonormal basis $\left\{\widetilde{u}_{j k}\right\}$ of $L^{2}(K)$ of eigenfunctions

$$
\widetilde{u}_{j k}\left(x^{\prime}, x^{\prime \prime}\right)=\widetilde{u}_{j}^{\prime}\left(x^{\prime}\right) \widetilde{u}_{k}^{\prime \prime}\left(x^{\prime \prime}\right)
$$

with

$$
-\widetilde{\Delta} \widetilde{u}_{j k}=\left(\widetilde{\lambda}_{j}^{\prime}+\widetilde{\lambda}_{k}^{\prime \prime}\right) \widetilde{u}_{j k} .
$$

The analog of Theorem 2.6 says that dom $\widetilde{\mathcal{E}}$ is characterized by

$$
\sum_{j} \sum_{k}\left(\widetilde{\lambda}_{j}^{\prime}+\widetilde{\lambda}_{k}^{\prime \prime}\right) a_{j k}(u)^{2}=\widetilde{\mathcal{E}}(u, u)<\infty .
$$

The proof is essentially the same.

Theorem 5.1. $u \in \operatorname{dom}_{L^{2}} \widetilde{\Delta}$ if and only if

$$
\sum_{j} \sum_{k}\left(\widetilde{\lambda}_{j}^{\prime}+\widetilde{\lambda}_{k}^{\prime \prime}\right)^{2} a_{j k}(u)^{2}<\infty
$$

in which case the left side of (5.7) gives $\|\widetilde{\Delta} u\|_{2}^{2}$ and

$$
-\widetilde{\Delta} u=\sum_{j} \sum_{k}\left(\widetilde{\lambda}_{j}^{\prime}+\widetilde{\lambda}_{k}^{\prime \prime}\right) a_{j k}(u) \widetilde{u}_{j k} .
$$

Proof. If the expansion is finite, it is easy to see (5.8) and the equality of the left side of (5.7) and $\|\widetilde{\Delta} u\|_{2}^{2}$ by direct computation. It is then routine to extend the result to all expansions when (5.7) holds.

Conversely, suppose $u \in \operatorname{dom}_{L^{2}} \widetilde{\Delta}$ and $\widetilde{\Delta} u=f$, with $f \in L^{2}$. So both $u$ and $f$ have expansions, and they are related. Since both $u$ and $\widetilde{u}_{j k}$ are in dom $\widetilde{\mathcal{E}}$, we use the definition of $\widetilde{\Delta}$ in two ways:

$$
-\widetilde{\mathcal{E}}\left(u, \widetilde{u}_{j k}\right)=\int(\widetilde{\Delta} u) \widetilde{u}_{j k} d \mu=a_{j k}(f)
$$

and

$$
-\widetilde{\mathcal{E}}\left(\widetilde{u}_{j k}, u\right)=\int\left(\widetilde{\Delta}_{j k}\right) u d \mu=-\left(\widetilde{\lambda}_{j}^{\prime}+\widetilde{\lambda}_{j}^{\prime \prime}\right) a_{j k}(u) .
$$

This proves (5.8), and since $f \in L^{2}$ we obtain (5.7).

Corollary 5.2. dom $_{L^{2}} \widetilde{\Delta}$ modulo constants is a Hilbert space, and it is dense in dom $\widetilde{\mathcal{E}}$ modulo constants.

The next result could be expressed succinctly as $\operatorname{dom}_{L^{2}} \widetilde{\Delta}=\operatorname{dom}_{L^{2}} \widetilde{\Delta}^{\prime} \cap \operatorname{dom}_{L^{2}} \widetilde{\Delta}^{\prime \prime}$.

Theorem 5.3. $u \in \operatorname{dom}_{L^{2}} \widetilde{\Delta}$ if and only if for almost every $x^{\prime \prime}, u\left(\cdot, x^{\prime \prime}\right) \in \operatorname{dom}_{L^{2}} \widetilde{\Delta}^{\prime}$ and $\widetilde{\Delta}^{\prime} u\left(\cdot, x^{\prime \prime}\right)=f_{1}\left(x^{\prime}, x^{\prime \prime}\right)$ with $f_{1} \in L^{2}$, and also for almost every $x^{\prime}, u\left(x^{\prime}, \cdot\right) \in$ 
$\operatorname{dom}_{L^{2}} \widetilde{\Delta}^{\prime \prime}$ and $\widetilde{\Delta}^{\prime \prime} u\left(x^{\prime}, \cdot\right)=f_{2}\left(x^{\prime}, x^{\prime \prime}\right)$ with $f_{2} \in L^{2}$. In that case

$$
\left\{\begin{array}{l}
f_{1}=-\sum_{j} \sum_{k} \widetilde{\lambda}_{j}^{\prime} a_{j k}(u) \widetilde{u}_{j k}, \\
f_{2}=-\sum_{j} \sum_{k} \widetilde{\lambda}_{k}^{\prime \prime} a_{j k}(u) \widetilde{u}_{j k},
\end{array}\right.
$$

so

$$
\widetilde{\Delta} u=f_{1}+f_{2} .
$$

Also

$$
\left\|f_{1}\right\|_{2}^{2}+\left\|f_{2}\right\|_{2}^{2} \leq\|\widetilde{\Delta} u\|_{2}^{2} \leq 2\left(\left\|f_{1}\right\|_{2}^{2}+\left\|f_{2}\right\|_{2}^{2}\right) .
$$

Proof. Suppose $u\left(\cdot, x^{\prime \prime}\right) \in \operatorname{dom}_{L^{2}} \widetilde{\Delta}^{\prime}$ and $\widetilde{\Delta}^{\prime} u\left(\cdot, x^{\prime \prime}\right)=f_{1}\left(x^{\prime}, x^{\prime \prime}\right)$ for a fixed $x^{\prime \prime}$. Then

$$
-\widetilde{\lambda}_{j}^{\prime} \int u \widetilde{u}_{j k} d \mu^{\prime}=-\widetilde{\mathcal{E}}^{\prime}\left(u\left(\cdot, x^{\prime \prime}\right), \widetilde{u}_{j k}\right)=\int_{\widetilde{K}^{\prime}} f_{1} \widetilde{u}_{j k} d \mu^{\prime} .
$$

If this holds for almost every $x^{\prime \prime}$ and $f_{1} \in L^{2}$, then we may integrate with respect to $x^{\prime \prime}$ to obtain $-\widetilde{\lambda}_{j}^{\prime} a_{j k}(u)=a_{j k}\left(f_{1}\right)$. In this way we establish (5.9). Then (5.10) and the right inequality in (5.11) follow.

Conversely, suppose $u \in \operatorname{dom}_{L^{2}} \widetilde{\Delta}$. Use (5.9) to define $f_{1}$ and $f_{2}$. Then $f_{1}$ and $f_{2}$ are in $L^{2}$ and the left inequality in (5.11) holds. For almost every $x^{\prime \prime}$, the function $f_{1}\left(\cdot, x^{\prime \prime}\right) \in L^{2}\left(\widetilde{K}^{\prime}\right)$. For such $x^{\prime \prime}$ it is easy to see that $\widetilde{\Delta}^{\prime} u\left(\cdot, x^{\prime \prime}\right)=f_{1}\left(x^{\prime}, x^{\prime \prime}\right)$.

It follows easily that the "no squares" theorem of [BST] extends from $K^{\prime}$ to $\widetilde{K}$ : if $u \in \operatorname{dom}_{L^{2}} \widetilde{\Delta}$ is nonconstant, then $u^{2} \notin \operatorname{dom}_{L^{2}} \widetilde{\Delta}$.

We may also express the result in terms of "Riesz transforms". Define $R^{\prime} u$ by

$$
a_{j k}\left(R^{\prime} u\right)=\frac{\widetilde{\lambda}_{j}^{\prime}}{\widetilde{\lambda}_{j}^{\prime}+\widetilde{\lambda}_{k}^{\prime \prime}} a_{j k}(u)
$$

and similarly for $R^{\prime \prime}$. Then

$$
f_{1}=R^{\prime}(\widetilde{\Delta} u), \quad f_{2}=R^{\prime \prime}(\widetilde{\Delta} u) .
$$

The point of Theorem 5.3 is that the Riesz transforms are bounded on $L^{2}$, and $\|u\|_{2}^{2} \approx\left\|R^{\prime} u\right\|_{2}^{2}+\left\|R^{\prime \prime} u\right\|_{2}^{2}$, which are in fact obvious from (5.12). We believe that they are also bounded on all $L^{q}, 1<q<\infty$. This is well known for the torus, and should be true quite generally using methods in DOS. In the case of the torus it is also well known that the Riesz transforms do not preserve the space of continous functions, and there is no reason to expect any better behavior in the fractal case. That means we emphatically do not expect an analog of the theorem to hold for $\operatorname{dom} \widetilde{\Delta}$. Since $\operatorname{dom} \widetilde{\Delta} \subseteq \operatorname{dom}_{L^{2}} \widetilde{\Delta}$, however, we can still use the theorem to obtain information about functions in dom $\widetilde{\Delta}$.

One important application of the theorem is to prove the existence of normal derivatives and the Gauss-Green formula. First we comment on the situation on $K^{\prime}$. The usual formulation is to assume the function belongs to dom $\Delta^{\prime}$, but we claim that it suffices to deal with functions in $\operatorname{dom}_{L^{2}} \Delta^{\prime}$. The point is that such functions can be represented as

$$
u^{\prime}\left(x^{\prime}\right)=-\int_{K^{\prime}} G^{\prime}\left(x^{\prime}, y^{\prime}\right)\left(\Delta^{\prime} u^{\prime}\left(y^{\prime}\right)\right) d \mu^{\prime}\left(y^{\prime}\right)+h^{\prime}\left(x^{\prime}\right),
$$


where $G^{\prime}$ is the Green's function for $K^{\prime}$ and $h^{\prime}$ is harmonic. Of course the harmonic function has normal derivatives at all boundary points, and we may differentiate (5.14) to obtain

$$
\partial_{n} u^{\prime}\left(q_{i}^{\prime}\right)=\int \partial_{n} G^{\prime}\left(q_{i}^{\prime}, y^{\prime}\right)\left(\Delta^{\prime} u^{\prime}\left(y^{\prime}\right)\right) d \mu^{\prime}\left(y^{\prime}\right)+\partial_{n} h^{\prime}\left(q_{i}^{\prime}\right)
$$

(note that $\partial_{n} G^{\prime}\left(q_{i}^{\prime}, y^{\prime}\right)$ is a harmonic function of $y^{\prime}$, in fact $\left.\partial_{n} G^{\prime}\left(q_{i}^{\prime}, q_{j}^{\prime}\right)=-\delta_{i j}\right)$. In fact we even have the estimate

$$
\left|\partial_{n} u^{\prime}\left(q_{i}^{\prime}\right)\right| \leq c\left(\left\|\Delta^{\prime} u^{\prime}\right\|_{2}+\max _{\partial K^{\prime}}\left|u^{\prime}\right|\right),
$$

since $h^{\prime}\left(q_{i}^{\prime}\right)=u\left(q_{i}^{\prime}\right)$. It is then easy to extend the two versions of Gauss-Green as follows:

i) if $u^{\prime} \in \operatorname{dom}_{L^{2}} \Delta^{\prime}$ and $v^{\prime} \in \operatorname{dom} \mathcal{E}^{\prime}$, then

$$
-\mathcal{E}^{\prime}\left(u^{\prime}, v^{\prime}\right)=\int_{K^{\prime}}\left(\Delta^{\prime} u^{\prime}\right) v^{\prime} d \mu^{\prime}-\sum_{\partial K^{\prime}}\left(\partial_{n}^{\prime} u^{\prime}\right) v^{\prime} ;
$$

ii) if $u^{\prime}$ and $v^{\prime}$ are in $\operatorname{dom}_{L^{2}} \Delta^{\prime}$, then

$$
\int_{K^{\prime}}\left(\left(\Delta^{\prime} u^{\prime}\right) v^{\prime}-u^{\prime} \Delta v^{\prime}\right) d \mu^{\prime}=\sum_{\partial K^{\prime}}\left(\left(\partial_{n}^{\prime} u^{\prime}\right) v^{\prime}-u^{\prime} \partial_{n} v^{\prime}\right) .
$$

Moreover, we may localize to any cell in $K^{\prime}$, and obtain similar results on cells in $\widetilde{K}^{\prime}$.

Now suppose $C$ is any cell in $K$, which we may regard as a subset of $\widetilde{K}$, say $C=F_{w} K$. Then $\partial C$ can be regarded as consisting of "horizontal" pieces of the form $\left\{\left(F_{w^{\prime}}^{\prime} x^{\prime}, F_{w^{\prime \prime}}^{\prime \prime} q_{i}^{\prime \prime}\right)\right\}$ and "vertical" pieces of the form $\left\{\left(F_{w^{\prime}}^{\prime} q_{j}^{\prime}, F_{w^{\prime \prime}}^{\prime \prime} x^{\prime \prime}\right)\right\}$. There are points in common, but these are isolated and have measure zero. On the horizontal pieces we seek normal derivatives of the form $\partial_{n}^{\prime \prime} u\left(F_{w^{\prime}}^{\prime} x^{\prime}, F_{w^{\prime \prime}}^{\prime \prime} q_{i}^{\prime \prime}\right)$ and on vertical pieces $\partial_{n}^{\prime} u\left(F_{w^{\prime}}^{\prime} q_{j}^{\prime}, F_{w^{\prime \prime}}^{\prime \prime} x^{\prime \prime}\right)$. By $\partial_{n} u$ we denote the appropriate choice without specifying the details. Note that $\partial_{n} u$ is ambiguously defined at the common points. There is a natural measure $\nu$ on $\partial C$, using $d \mu^{\prime}$ on horizontal pieces and $d \mu^{\prime \prime}$ on vertical pieces. A similar description holds if $C$ is a simple subset, since the boundary of $C$ is a subset of the boundaries of the cells in $C$.

Theorem 5.4. (a) Let $u \in \operatorname{dom}_{L^{2}} \widetilde{\Delta}$. Then $\partial_{n} u$ is defined almost everywhere on $\partial C$, and $\partial_{n} u \in L^{2}(\partial C)$.

(b) If $u \in \operatorname{dom}_{L^{2}} \widetilde{\Delta}$ and $v \in \operatorname{dom} \widetilde{\mathcal{E}}$, then

$$
-\widetilde{\mathcal{E}}_{C}(u, v)=\int_{C}(\widetilde{\Delta} u) v d \mu-\int_{\partial C}\left(\partial_{n} u\right) v d \nu .
$$

(c) If $u, v \in \operatorname{dom}_{L^{2}} \widetilde{\Delta}$, then

$$
\int_{C}((\widetilde{\Delta} u) v-u \widetilde{\Delta} v) d \mu=\int_{\partial C}\left(\left(\partial_{n} u\right) v-u \partial_{n} v\right) d \nu .
$$

Proof. (a) Theorem 5.3 and the above discussion show that $\partial_{n} u$ exists almost everywhere in $\partial C$. Moreover the estimate (5.16) shows that $\partial_{n} u \in L^{2}(\partial C)$, since we already know that $u \in \operatorname{dom} \widetilde{\mathcal{E}}$ implies the restriction of $u$ to $\partial C$ is in $L^{2}$.

(b) Take the localized verison of (5.17) on $F_{w^{\prime}}^{\prime} K^{\prime}$ and integrate with respect to $x^{\prime \prime}$ over $F_{w^{\prime \prime}}^{\prime \prime} K^{\prime \prime}$. Do the same in the reverse order and add to obtain (5.19). Here we 
use Theorem 5.3 to control sections of $u$, and the corresponding fact for $v \in \operatorname{dom} \widetilde{\mathcal{E}}$; namely, for almost every $x^{\prime \prime}, v\left(\cdot, x^{\prime \prime}\right) \in \operatorname{dom} \widetilde{\mathcal{E}}^{\prime}$ and

$$
\int \widetilde{\mathcal{E}}^{\prime}\left(v\left(\cdot, x^{\prime \prime}\right), v\left(\cdot, x^{\prime \prime}\right)\right) d \mu\left(x^{\prime \prime}\right) \leq \widetilde{\mathcal{E}}(v, v)
$$

(c) By subtracting two versions of (5.19) with $u$ and $v$ reversed we obtain (5.20).

We now discuss some of the relationships between functions on $K$ and $\widetilde{K}$. We write $E R(f)$ and $O R(f)$ for the even and odd reflections of a function $f$ defined on $K$,

$$
E R(f)\left(x^{\prime}, x^{\prime \prime}\right)=E R(f)\left(-x^{\prime}, x^{\prime \prime}\right)=E R(f)\left(x^{\prime},-x^{\prime \prime}\right)=E R(f)\left(-x^{\prime},-x^{\prime \prime}\right)
$$

and

$$
\begin{aligned}
O R(f)\left(x^{\prime}, x^{\prime \prime}\right) & =-O R(f)\left(-x^{\prime}, x^{\prime \prime}\right)=-O R(f)\left(x^{\prime},-x^{\prime \prime}\right) \\
& =O R(f)\left(-x^{\prime},-x^{\prime \prime}\right) .
\end{aligned}
$$

Both operations preserve $L^{2}$, and ER preserves continuous functions, while $O R(f)$ is continuous if and only if $f$ is continuous and vanishes on $\partial K$.

Lemma 5.5. (a) $v \in \operatorname{dom} \mathcal{E}$ if and only if $E R(v) \in \operatorname{dom} \widetilde{\mathcal{E}}$. (b) $v \in d_{0} m_{0} \mathcal{E}$ if and only if $\operatorname{OR}(v) \in \operatorname{dom} \widetilde{\mathcal{E}}$.

Proof. Note that the same statements are true for $K^{\prime}$ and $\widetilde{K}^{\prime}$. The extension to the product situation is routine.

Theorem 5.6. $u \in$ dom $_{L^{2}} \Delta$ with $\Delta u=f$ and $\left.u\right|_{\partial K}=0$ a.e. if and only if $O R(u) \in \operatorname{dom}_{L^{2}} \widetilde{\Delta}$ with $\widetilde{\Delta} O R(u)=O R(f)$. In particlar, Theorem 5.4 holds for such functions.

Proof. Assume $u \in \operatorname{dom}_{L^{2}} \Delta$ with $\Delta u=f$ and $\left.u\right|_{\partial K}=0$ a.e. Note that every $\widetilde{v} \in \operatorname{dom} \widetilde{\mathcal{E}}$ may be written as a sum of four functions with parity types even-even, even-odd, odd-even and odd-odd. Now the vanishing of $u$ on the boundary means $u \in \operatorname{dom}_{0} \mathcal{E}$, hence $O R(u) \in \operatorname{dom} \widetilde{\mathcal{E}}$ by Lemma 5.5 (b). Thus it suffices to show that

$$
-\widetilde{\mathcal{E}}(O R(u), \widetilde{v})=\int_{\widetilde{K}} O R(f) \widetilde{v} d \mu
$$

for $\widetilde{v} \in \operatorname{dom} \widetilde{\mathcal{E}}$ of each of the four parity types. But both sides of (5.23) vanish in the first three cases by parity considerations, while in the odd-odd case we have $-\widetilde{\mathcal{E}}(O R(u), \widetilde{v})=-4 \mathcal{E}(u, v)$ and $\int_{\widetilde{K}} O R(f) \widetilde{v} d \mu=4 \int_{k} f v d \mu$ for $v=\left.\widetilde{v}\right|_{K}$. But also $\widetilde{v}=O R(v)$, so $v \in \operatorname{dom}_{0} \mathcal{E}$ in this case, so $-\mathcal{E}(u, v)=\int_{K} f v d \mu$ which proves (5.23). The proof of the converse is similar.

Note that if $u \in \operatorname{dom} \Delta$ and $\left.u\right|_{\partial K}=0$ a.e. we cannot conclude that $O R(u) \in$ $\operatorname{dom} \widetilde{\Delta}$ because $O R(f)$ need not be continuous.

Functions satisfying the hypotheses of Theorem 5.6 may be said to belong to the Dirichlet domain of $\Delta$. Similarly, a function $u$ is said to belong to the Neumann domain of $\Delta$ with $\Delta u=f$ for $f \in L^{2}(K)$ if $u \in \operatorname{dom} \mathcal{E}$ and

$$
-\mathcal{E}(u, v)=\int_{K} f v d \mu \text { for all } v \in \operatorname{dom} \mathcal{E} \text {. }
$$


Theorem 5.7. $u$ is in the Neumann domain of $\Delta$ if and only if $E R(u) \in \operatorname{dom}_{L^{2}} \widetilde{\Delta}$. Moreover, for such functions $\partial_{n} u=0$ almost everywhere on $\partial K$.

Proof. The equivalence is proved by similar arguments to the proof of Theorem 5.6 , only now $\widetilde{\Delta} E R(u)=E R(f)$ and only the even-even parity type for $\widetilde{v}$ produces nonzero values. If $E R(u) \in \operatorname{dom}_{L^{2}} \widetilde{\Delta}$ we may apply Theorem 5.4 to $K$. In particular, $\partial_{n} u$ exists almost everywhere on $\partial K$, and by (5.19) we have

$$
-\mathcal{E}(u, v)=\int_{K} f v d \mu-\int_{\partial K}\left(\partial_{n} u\right) v d \nu \text { for every } v \in \operatorname{dom} \mathcal{E} .
$$

Combined with (5.24) this implies $\int_{\partial K}\left(\partial_{n} u\right) v d \nu=0$. Since the boundary values of $v$ are dense in $L^{2}(\partial K)$, it follows that $\partial_{n} u=0$ almost everywhere.

We expect that conversely $\partial_{n} u=0$ a.e. and $u \in \operatorname{dom}_{L^{2}} \Delta$ should imply that $u$ is in the Neumann domain of $\Delta$, but it is not clear how to prove this.

The next result is a "weak $=$ strong" theorem on $\widetilde{K}$.

Theorem 5.8. Suppose $u$ and $f$ are in $L^{2}(\widetilde{K})$. Then for all $v \in \operatorname{dom}_{L^{2}} \widetilde{\Delta}$ we have

$$
\int_{\widetilde{K}} u \widetilde{\Delta} v d \mu=\int_{\widetilde{K}} f v d \mu,
$$

if and only if $u \in \operatorname{dom}_{L^{2}} \widetilde{\Delta}$ and $\widetilde{\Delta} u=f$. If, in addition, $f \in C(\widetilde{K})$, then $u \in \operatorname{dom} \widetilde{\Delta}$.

Proof. Note that (5.25) holds by Theorem 5.4 (c) if $u \in \operatorname{dom}_{L^{2}} \widetilde{\Delta}$ and $\widetilde{\Delta} u=f$. Conversely, suppose (5.25) holds. Since both $u$ and $f$ are in $L^{2}$, we have eigenfunction expansions

$$
\begin{aligned}
& u=\sum_{j} \sum_{k} a_{j k}(u) \widetilde{u}_{j k}, \\
& f=\sum_{j} \sum_{k} a_{j k}(f) \widetilde{u}_{j k} .
\end{aligned}
$$

To complete the proof it suffices to show $\left(\widetilde{\lambda}_{j}^{\prime}+\widetilde{\lambda}_{k}^{\prime}\right) a_{j k}(u)=a_{j k}(f)$ by Theorem 5.1. But this follows from (5.25) with the choice $v=\widetilde{u}_{j k}$.

It would be very desirable to establish a local version of this result. For example, this could be used to show that uniform limits of harmonic functions are harmonic.

\section{Heat kernel estimates}

In this section we observe that it is easy to combine heat kernel estimates on $K^{\prime}$ and $K^{\prime \prime}$ to obtain heat kernel estimates on $K$. These estimates have important consequences. We can work equally well either on $K^{\prime}$ with Dirichlet or Neumann conditions, or on $\widetilde{K}^{\prime}$ without boundary conditions. For simplicity we work on the cover $\widetilde{K}^{\prime}$, since the other cases just involve taking odd and even parts (we are not concerned here with lower bounds, which have not been established in the case of Dirichlet boundary conditions). The heat kernel $\widetilde{p}_{t}^{\prime}\left(x^{\prime}, y^{\prime}\right)$ in $\widetilde{K}^{\prime}$ may be defined by the eigenfunction expansion

$$
\widetilde{p}_{t}^{\prime}\left(x^{\prime}, y^{\prime}\right)=\sum_{j=0}^{\infty} e^{-t \widetilde{\lambda}_{j}^{\prime}} \widetilde{u}_{j}^{\prime}\left(x^{\prime}\right) \widetilde{u}_{j}^{\prime}\left(y^{\prime}\right) .
$$


We may solve the heat equation

$$
\frac{\partial}{\partial t}\left(x^{\prime}, t\right)=\widetilde{\Delta}^{\prime} u\left(x^{\prime}, t\right), \quad t>0,
$$

with initial conditions

$$
u\left(x^{\prime}, 0\right)=f\left(x^{\prime}\right)
$$

for any $f \in L^{2}\left(\widetilde{K}^{\prime}\right)$ via

$$
u\left(x^{\prime}, t\right)=\int_{\widetilde{K}^{\prime}} \widetilde{p}_{t}\left(x^{\prime}, y^{\prime}\right) f\left(y^{\prime}\right) d \mu^{\prime}\left(y^{\prime}\right) .
$$

Symbolically, we write $u=e^{t \widetilde{\Delta}^{\prime}} f$.

To describe the estimates on this heat kernel we first need to discuss the effective resistance metric $d^{\prime}\left(x^{\prime}, y^{\prime}\right)$ on $\widetilde{K}^{\prime}$. This is defined by

$$
d^{\prime}\left(x^{\prime}, y^{\prime}\right)=\left(\inf \left\{\widetilde{\mathcal{E}}^{\prime}(h, h): h\left(x^{\prime}\right)=0, h\left(y^{\prime}\right)=1\right\}\right)^{-1} .
$$

The function that achieves the minimum in (6.5) is harmonic in the complement of the points $x^{\prime}$ and $y^{\prime}$. In this metric each cell $F_{w^{\prime}}^{\prime} K^{\prime}$ has diameter on the order of $r_{w^{\prime}}^{\prime}$. In order to have a uniform relationship between measure and metric we will assume

$$
\mu_{i}^{\prime}=\left(r_{i}^{\prime}\right)^{d^{\prime}} \text { for all } i
$$

for some fixed $d^{\prime}$, which we will call the dimension of $K^{\prime}$ (more precisely, it is the dimension of the measure $\left.\mu^{\prime}\right)$. Then

$$
\mu^{\prime}\left(B_{r}\right) \sim r^{d^{\prime}},
$$

where $B_{r}$ denotes any ball of radius $r$ in the metric. Since the relation (6.6) tends to clash with the assumptions of Lemma 2.2 , the only interesting examples will have all $r_{i}^{\prime}$ the same. In the case SG the dimension is $\log 3 / \log (5 / 3)$.

The heat kernel estimate is that $\widetilde{p}_{t}^{\prime}\left(x^{\prime}, y^{\prime}\right)$ is bounded above and below by a multiple of

$$
t^{-\frac{d^{\prime}}{d^{\prime}+1}} \exp \left(-c_{2}\left(\frac{d^{\prime}\left(x^{\prime}, y^{\prime}\right)^{d^{\prime}+1}}{t}\right)^{J}\right), \text { for } 0<t<1,
$$

for a constant $J>0$ that depends on $K^{\prime}$. This is proved for $K^{\prime}=\mathrm{SG}$ (with $J=\log 2 / \log (5 / 2))$ in $[\mathrm{BP}$ and for a wider class of fractals in [HK]. For the rest of the paper we assume that (6.8) holds for $\widetilde{K}^{\prime}$, and $K^{\prime \prime}=K^{\prime}$. We will also use the trivial estimate that $\widetilde{p}_{t}^{\prime}\left(x^{\prime}, y^{\prime}\right)$ is uniformly bounded in $t \geq 1$, and also $\widetilde{p}_{t}^{\prime}$ is continuous. In [S5] it is observed that such heat kernel estimates are consistent with the idea that $\widetilde{\Delta}^{\prime}$ is an operator of order $d^{\prime}+1$. Unfortunately, an incorrect value for $J$ is given in [S5]. However, this error does not have significant consequences.

We introduce a metric on $\widetilde{K}=\widetilde{K}^{\prime} \times \widetilde{K}^{\prime}$ by

$$
d(x, y)=\left(d^{\prime}\left(x^{\prime}, y^{\prime}\right)^{J\left(d^{\prime}+1\right)}+d^{\prime}\left(x^{\prime \prime}, y^{\prime \prime}\right)^{J\left(d^{\prime}+1\right)}\right)^{\frac{1}{J\left(d^{\prime}+1\right)}}
$$

for $x=\left(x^{\prime}, x^{\prime \prime}\right)$ and $y=\left(y^{\prime}, y^{\prime \prime}\right)$. In this metric we have the analog of (6.7) with $d^{\prime}$ replaced by $2 d^{\prime}$, so it is natural to think of $2 d^{\prime}$ as the dimension of $\widetilde{K}$.

The heat kernel $\widetilde{p}_{t}(x, y)$ on $\widetilde{K}$ may be defined by the eigenfunction expansion

$$
\tilde{p}_{t}(x, y)=\sum_{j=0}^{\infty} \sum_{k=0}^{\infty} e^{-t\left(\widetilde{\lambda}_{j}^{\prime}+\tilde{\lambda}_{k}^{\prime}\right)} \widetilde{u}_{j k}(x) \widetilde{u}_{j k}(y) .
$$


We may solve the heat equation

$$
\frac{\partial}{\partial t} u(x, t)=\widetilde{\Delta} u(x, t), \quad t>0,
$$

with initial conditions

$$
u(x, 0)=f(x)
$$

for any $f \in L^{2}(\widetilde{K})$ via

$$
u(x, t)=\int_{\widetilde{K}} \widetilde{p}_{t}(x, y) f(y) d \mu(y) .
$$

Theorem 6.1. The heat kernel on $\widetilde{K}$ factors as

$$
\widetilde{p}_{t}(x, y)=\widetilde{p}_{t}^{\prime}\left(x^{\prime}, y^{\prime}\right) \widetilde{p}_{t}^{\prime}\left(x^{\prime \prime}, y^{\prime \prime}\right) .
$$

It satisfies the estimate that $\widetilde{p}_{t}(x, y)$ is bounded above and below by a multiple of

$$
t^{-\frac{2 d^{\prime}}{d^{\prime}+1}} \exp \left(-c_{2}\left(\frac{d(x, y)^{d^{\prime}+1}}{t}\right)^{J}\right) \text { for } 0<t<1 .
$$

Proof. (6.14) follows immediately from (6.10). Then (6.15) follows using (6.8) and (6.9).

We continue to regard $\widetilde{\Delta}$ as an operator of order $d^{\prime}+1$. The explanation of the exponents in (6.8) and (6.15) is that the power of $t$ is -dimension/order, and the powers inside the exponential are order and $1 /($ order -1$)$. This appears to be consistent with other known estimates [B], [BD].

An immediate consequence of heat kernel estimates are Sobolev embedding theorems [C], GHL]. We define the Sobolev spaces $L_{s}^{p}$ for $1<p<\infty$ and $s \geq 0$ to be the image of $L^{p}$ under the operator $(I-\widetilde{\Delta})^{-\frac{s}{d^{\prime}+1}}$. Then we may identify dom $\widetilde{\mathcal{E}}$ with $L_{\frac{d^{\prime}+1}{2}}^{2}$ and $\operatorname{dom}_{L^{2}} \widetilde{\Delta}$ with $L_{d^{\prime}+1}^{2}$.

Corollary 6.2. (a) If $s<2 d^{\prime} / p$, then $L_{s}^{p} \subseteq L^{q}$ for $\frac{1}{q}=\frac{1}{2}-\frac{s}{2 d^{\prime}}$. (b) If $s>2 d^{\prime} / p$, then $L_{s}^{p} \subseteq C$. (c) In particular dom $\widetilde{\mathcal{E}} \subseteq L^{q}$ for $q=\frac{4 d^{\prime}}{d^{\prime}-1}$ and $\operatorname{dom}_{L^{2}} \widetilde{\Delta} \subseteq C$.

Note that Theorem 2.4 shows that the embedding of dom $\widetilde{\mathcal{E}}$ is sharp, since (2.6) says exactly $q>\frac{4 d^{\prime}}{d^{\prime}-1}$. If we took an $n$-fold product of $\widetilde{K}$ with $n>2$, then we would no longer have $\operatorname{dom}_{L^{2}} \widetilde{\Delta} \subseteq C$, since the condition would be $s>n d^{\prime} / p$. However, since dom $\widetilde{\Delta} \subseteq L_{d^{\prime}+1}^{p}$ for any $p<\infty$, we will always have dom $\widetilde{\Delta} \subseteq C$.

The embedding of dom $\widetilde{\mathcal{E}}$ implies that we could extend the definition of $\operatorname{dom}_{L^{q}} \Delta$ down to $q \geq \frac{4 d^{\prime}}{3 d^{\prime}+1}$. For the $n$-fold product the bound is $q \geq \frac{2 n d^{\prime}}{(n+1) d^{\prime}+1}$.

The embedding of $L_{s}^{2}$ in $L_{t}^{2}$ for $s>t$ is easily seen to be compact. (We believe the same is true with 2 replaced by any $p$.) Combined with Corollary 6.2, this leads to many other compact embeddings.

The embedding in Corollary 6.2 (b) can be refined to an embedding into Hölder spaces. Because of technicalities we will not discuss such results here.

Another important, and closely related, consequence of heat kernel estimates is an estimate for Green's functions. First we discuss $\widetilde{K}$. A convenient way to factor out constants is to work with functions having total integral zero. Let

$$
\widetilde{G}(x, y)=\sum_{(j, k) \neq(0,0)} \frac{1}{\widetilde{\lambda}_{j}^{\prime}+\widetilde{\lambda}_{k}^{\prime}} \widetilde{u}_{j k}(x) \widetilde{u}_{j k}(y)
$$


If $f \in L^{2}(\widetilde{K})$ with $\int_{\widetilde{K}} f d \mu=0$, then

$$
u(x)=\int_{\widetilde{K}} \widetilde{G}(x, y) f(y) d \mu
$$

gives the unique solution of $-\widetilde{\Delta} u=f$ with $\int_{\widetilde{K}} u d \mu=0$.

Corollary 6.3. Suppose $d^{\prime}>1$. Then

$$
\widetilde{G}(x, y) \leq c d(x, y)^{1-d^{\prime}} .
$$

Proof. We have

$$
\widetilde{G}(x, y)=\int_{0}^{\infty}\left(\widetilde{p}_{t}(x, y)-1\right) d t .
$$

For $0<t<1$ we use $(6.15)$ to obtain

$$
\int_{0}^{1} \widetilde{p}_{t}(x, y) d t \leq c d(x, y)^{1-d^{\prime}} .
$$

For $t \geq 1$ we use routine estimates to show $\widetilde{p}_{t}(x, y)-1$ decays exponentially in $t$ so $\int_{1}^{\infty} \widetilde{p}_{t}(x, y) d t$ is bounded.

Similarly, we define a Dirichlet Green's function $G_{D}(x, y)$ on $K \times K$ by

$$
G_{D}(x, y)=\sum_{j=1}^{\infty} \sum_{k=1}^{\infty} \frac{1}{\lambda_{j, D}^{\prime}+\lambda_{k, D}^{\prime}} u_{j k, D}(x) u_{j k, D}(y),
$$

where

$$
u_{j k, D}(x)=u_{j, D}^{\prime}\left(x^{\prime}\right) u_{k, D}^{\prime}\left(x^{\prime \prime}\right) .
$$

Then

$$
u(x)=\int_{K} G_{D}(x, y) f(y) d \mu(y)
$$

gives the unique solution of $-\Delta u=f$ with $\left.u\right|_{\partial K}=0$ for any $f \in L^{2}(K)$. The analog of (6.18) holds: if $d^{\prime}>1$, then

$$
\left|G_{D}(x, y)\right| \leq c d(x, y)^{1-d^{\prime}} .
$$

\section{TRACES AND EXTENSIONS}

In this section we continue the assumptions made in Section 6 , that $K^{\prime}=K^{\prime \prime}$, all $r_{i}^{\prime}$ are equal, all $\mu_{i}^{\prime}$ are equal, and $\mu_{i}^{\prime}=\left(r_{i}^{\prime}\right)^{d^{\prime}}$.

We begin by investigating the traces of functions in $L_{s}^{2}(\widetilde{K})$ on subsets obtained by fixing one of the variables, $x^{\prime}=y^{\prime}\left(\right.$ or $\left.x^{\prime \prime}=y^{\prime}\right)$ for $y^{\prime}$ fixed.

Theorem 7.1. Let $u \in L_{s}^{2}(\widetilde{K})$ for $s>d^{\prime} / 2$. Then for every fixed $y^{\prime}, \operatorname{Tr}_{y^{\prime}}(u)=$ $u\left(y^{\prime}, \cdot\right) \in L_{s-d^{\prime} / 2}^{2}\left(\widetilde{K}^{\prime}\right)$. In particular, if $u \in \operatorname{dom} \widetilde{\mathcal{E}}$, then $\operatorname{Tr}_{y^{\prime}}(u) \in L_{1 / 2}^{2}\left(\widetilde{K}^{\prime}\right)$, and if $u \in \operatorname{dom}_{L^{2}} \widetilde{\Delta}$, then $\operatorname{Tr}_{y^{\prime}}(u) \in L_{\frac{d^{\prime}}{2}+1}^{2}\left(\widetilde{K}^{\prime}\right)$.

Proof. For $u \in L_{s}^{2}(\widetilde{K})$ orthogonal to constants we have

$$
u\left(x^{\prime}, x^{\prime \prime}\right)=\sum_{j} \sum_{k} a_{j k}(u) \widetilde{u}_{j}\left(x^{\prime}\right) \widetilde{u}_{k}\left(x^{\prime \prime}\right)
$$


with

$$
\sum_{j} \sum_{k} a_{j k}(u)^{2}\left(\widetilde{\lambda}_{j}^{\prime}+\widetilde{\lambda}_{k}^{\prime}\right)^{\frac{2 s}{d^{\prime}+1}}=\|u\|_{L_{s}^{2}(\widetilde{K})}^{2}
$$

Then, at least formally,

$$
\operatorname{Tr}_{y^{\prime}}(u)=\sum_{k}\left(\sum_{j} a_{j k}(u) \widetilde{u}_{j}\left(y^{\prime}\right)\right) \widetilde{u}_{k}\left(x^{\prime \prime}\right),
$$

so it suffices to show that

$$
\sum_{k}\left(\sum_{j} a_{j k}(u) \widetilde{u}_{j}\left(y^{\prime}\right)\right)^{2}\left(\widetilde{\lambda}_{k}^{\prime}\right)^{\frac{2 s-d^{\prime}}{d^{\prime}+1}} \leq c\|u\|_{L_{s}^{2}(\widetilde{K})}^{2} .
$$

We will do this by using the Cauchy-Schwartz inequality as follows:

$$
\begin{aligned}
& \left(\sum_{j} a_{j k}(u) \widetilde{u}_{j}\left(y^{\prime}\right)\right)^{2} \\
& \quad \leq\left(\sum_{j} a_{j k}(u)^{2}\left(\widetilde{\lambda}_{j}^{\prime}+\widetilde{\lambda}_{k}^{\prime}\right)^{\frac{2 s}{d^{\prime}+1}}\right)\left(\sum_{j}\left(\widetilde{\lambda}_{j}^{\prime}+\widetilde{\lambda}_{k}^{\prime}\right)^{-\frac{2 s}{d^{\prime}+1}} \widetilde{u}_{j}\left(y^{\prime}\right)^{2}\right) .
\end{aligned}
$$

We also observe that

$$
\sum_{j}\left(\widetilde{\lambda}_{j}^{\prime}+\widetilde{\lambda}_{k}^{\prime}\right)^{-\frac{2 s}{d^{\prime}+1}} \widetilde{u}_{j}\left(y^{\prime}\right)^{2}=\int_{0}^{\infty} \widetilde{p}_{t}^{\prime}\left(y^{\prime}, y^{\prime}\right) e^{-\tilde{\lambda}_{k}^{\prime} t} t^{\frac{2 s}{d^{\prime}+1}-1} d t .
$$

We use the heat kernel estimate (6.8) for $t \leq 1$ and the trivial uniform boundedness for $t \geq 1$ to obtain

$$
\begin{aligned}
\sum_{j}\left(\widetilde{\lambda}_{j}^{\prime}+\widetilde{\lambda}_{k}^{\prime}\right)^{-\frac{2 s}{d^{\prime}+1}} u_{j}\left(y^{\prime}\right)^{2} & \leq c \int_{0}^{\infty} e^{-\widetilde{\lambda}_{k}^{\prime} t} t^{\frac{2 s-d^{\prime}}{d^{\prime}+1}-1} d t+c \int_{1}^{\infty} e^{-\widetilde{\lambda}_{k}^{\prime} t} t^{\frac{2 s}{d^{\prime}+1}-1} d t \\
& \leq c\left(\left(\widetilde{\lambda}_{k}^{\prime}\right)^{-\left(\frac{2 s-d^{\prime}}{d^{\prime}+1}\right)}+e^{-\widetilde{\lambda}_{k}^{\prime}}\right) \\
& \leq c^{\prime}\left(\widetilde{\lambda}_{k}^{\prime}\right)^{-\left(\frac{2 s-d^{\prime}}{d^{\prime}+1}\right)}
\end{aligned}
$$

where the condition $s>d^{\prime} / 2$ guarantees the convergence of the first integral. Substituting this estimate in (7.5) and summing over $k$ yields (7.4).

We believe that the converse statement, every function in $L_{s-d^{\prime} / 2}^{2}\left(\widetilde{K}^{\prime}\right)$ is the trace of some $u \in L_{s}^{2}(\widetilde{K})$, is true in general, but we will only prove it in two cases of interest. In fact we are really interested in the trace on $\partial K$, which is a union of halves of subsets of the form considered above. The halving presents no difficulties, but there is the issue of the behavior in a neighborhood of the intersection points. For $s-d^{\prime} / 2$ small enough $\left(<d^{\prime} / 2\right)$ we do not have to require any consistency condition. For $s-d^{\prime} / 2>d^{\prime} / 2$ the functions in $L_{s-d^{\prime} / 2}^{2}\left(\widetilde{K}^{\prime}\right)$ are continuous, so we will also require functions in $L_{s-d^{\prime} / 2}^{2}(\partial K)$ to be continuous, and this is sufficient as long as $s-d^{\prime} / 2$ is not too large. The borderline case $s-d^{\prime} / 2=d^{\prime} / 2$ is more complicated; a complete description is given in [S1]. This is exactly the case for $\operatorname{dom} \mathcal{E}$ if $K$ is the square. Since this example is well understood, we will exclude it in what follows. 
Corollary 7.2. Suppose $d^{\prime}>1$. If $u \in \operatorname{dom} \mathcal{E}$, then $\operatorname{Tr}_{\partial K}(u) \in L_{1 / 2}^{2}(\partial K)$, where $L_{1 / 2}^{2}(\partial K)$ is defined by the requirement that on each subset of $\partial K$ of the form $q_{i}^{\prime} \times K^{\prime}$ or $K^{\prime} \times q_{i}^{\prime}$ the function belongs to $L_{1 / 2}^{2}\left(K^{\prime}\right)$ (the function can be extended to $\left.L_{1 / 2}^{2}\left(\widetilde{K}^{\prime}\right)\right)$.

Theorem 7.3. Suppose $d^{\prime}>1$. Then there exists a bounded linear extension operator $E: L_{1 / 2}^{2}(\partial K) \rightarrow \operatorname{dom} \mathcal{E}$ (extension means $\operatorname{Tr}_{\partial K} E \varphi=\varphi$ ).

Proof. It suffices to construct the extension when $\varphi$ vanishes on all of $\partial K$ except the face $K^{\prime} \times q_{i}^{\prime}$ for each boundary point $q_{i}^{\prime}$. Such a function has an expansion

$$
\varphi\left(x^{\prime}, q_{i}^{\prime}\right)=\sum_{j=1}^{\infty} a_{j}(\varphi) u_{j, D}^{\prime}\left(x^{\prime}\right)
$$

in Dirichlet eigenfunctions with

$$
\sum a_{j}(\varphi)^{2}\left(\lambda_{j, D}^{\prime}\right)^{\frac{1}{d^{\prime}+1}}=\|u\|_{L_{1 / 2}^{2}(\partial K)}^{2}<\infty .
$$

(This result is not quite established in [S5], where it is shown to be true modulo the finite-dimensional space of harmonic functions; in fact we do not need this because harmonic functions have an expansion of the form (7.6) because $1 / 2<d^{\prime} / 2$. However, it is trivial to define extensions for harmonic functions, so we do not really need this observation.)

We will take the extension operator to be of the form

$$
E \varphi\left(x^{\prime}, x^{\prime \prime}\right)=\sum_{j=1}^{\infty} a_{j}(\varphi) u_{j, D}^{\prime}\left(x^{\prime}\right) \psi_{j}\left(x^{\prime \prime}\right)
$$

where $\psi_{j}\left(q_{\ell}^{\prime}\right)=\delta_{i \ell}$ and the sum in (7.8) is finite for any $x^{\prime \prime} \neq q_{i}^{\prime}$. This condition guarantees $\operatorname{Tr}_{\partial K} E \varphi=\varphi$. We need further conditions on $\psi_{j}$ so that $E$ maps into $\operatorname{dom} \mathcal{E}$. The optimal choice of $\psi_{j}$, in the sense of minimizing $\mathcal{E}(E \varphi, E \varphi)$, would lead to $E \varphi$ being harmonic and $\psi_{j}$ being eigenfunctions (with eigenvalue $-\lambda_{j}^{\prime}$ ). We will discuss this in Section 8, but here we make a choice that leads to a simpler proof. Note that the orthogonality of $u_{j, D}^{\prime}$ with respect to both $\mathcal{E}^{\prime}$ and $L^{2}$ inner products means that

$$
\mathcal{E}(E \varphi, E \varphi)=\sum_{j=1}^{\infty} a_{j}(\varphi)^{2}\left(\mathcal{E}^{\prime}\left(\psi_{j}, \psi_{j}\right)+\lambda_{j, D}^{\prime}\left\|\psi_{j}\right\|_{2}^{2}\right) .
$$

In view of (7.7) it suffices to have

$$
\mathcal{E}^{\prime}\left(\psi_{j}, \psi_{j}\right) \leq c\left(\lambda_{j, D}^{\prime}\right)^{\frac{1}{d^{\prime}+1}}
$$

and

$$
\left\|\psi_{j}\right\|_{2}^{2} \leq c\left(\lambda_{j, D}^{\prime}\right)^{\frac{1}{d^{\prime}+1}-1} .
$$

We can accomplish this by taking $\psi_{j}$ to be the piecewise harmonic spline of level $m$ satisfying $\psi_{j}\left(q_{i}^{\prime}\right)=1$ and $\psi_{j}\left(x^{\prime}\right)=0$ for all $x^{\prime} \in V_{m}^{\prime} \backslash\left\{q_{i}^{\prime}\right\}$, where $m$ is chosen to depend on $j$. (Since we will have $m \rightarrow \infty$ as $j \rightarrow \infty$ this will make the sum (7.8) finite if $x^{\prime \prime} \neq q_{i}^{\prime}$.) Note that $\left\|\psi_{j}\right\|_{2}^{2}=c_{1}\left(\mu_{i}^{\prime}\right)^{m}$ and $\mathcal{E}\left(\psi_{j}, \psi_{j}\right)=c_{2}\left(r_{i}^{\prime}\right)^{-m}$. To satisfy (7.9) we choose $m$ to satisfy

$$
\left(r_{i}^{\prime}\right)^{-m} \leq\left(\lambda_{j, D}^{\prime}\right)^{\frac{1}{d^{\prime}+1}}<\left(r_{i}^{\prime}\right)^{-(m+1)} .
$$


Then

so (7.10) holds.

$$
\left(\mu_{i}^{\prime}\right)^{m}=\left(r_{i}^{\prime}\right)^{m d^{\prime}}<\left(r_{i}^{\prime}\right)^{-d^{\prime}}\left(\lambda_{j, D}^{\prime}\right)^{-\frac{d^{\prime}}{d^{\prime}+1}},
$$

Definition 7.4. The space $L_{\frac{d^{\prime}}{2}+1}^{2}(\partial K)$ is defined to be the space of continuous functions $\varphi$ on $\partial K$ whose restrictions to the subsets $K^{\prime} \times q_{i}^{\prime}$ and $q_{i}^{\prime} \times K^{\prime}$ belong to $L_{\frac{d^{\prime}}{2}+1}^{2}\left(K^{\prime}\right)$. Recall from S5 that

$$
L_{\frac{d^{\prime}}{2}+1}^{2}\left(K^{\prime}\right)=\mathcal{H}_{0}^{\prime} \oplus L_{\frac{d^{\prime}}{2}+1, D}^{2}\left(K^{\prime}\right)=\mathcal{H}_{0}^{\prime} \oplus L_{\frac{d^{\prime}}{2}+1, N}^{2}\left(K^{\prime}\right),
$$

where $\mathcal{H}_{0}^{\prime}$ is the space of harmonic functions on $K^{\prime}$, and $L_{\frac{d^{\prime}}{2}+1, D}^{2}\left(K^{\prime}\right)$ is the space of functions of the form

$$
\sum a_{j, D} u_{j, D}^{\prime}
$$

with

$$
\sum_{j=1}^{\infty}\left(\lambda_{j, D}^{\prime}\right)^{\frac{d^{\prime}+2}{d^{\prime}+1}}\left|a_{j, D}\right|^{2}<\infty
$$

and similarly for $L_{\frac{d^{\prime}}{2}+1, N}^{2}\left(K^{\prime}\right)$ with $u_{j, N}^{\prime}$ replacing $u_{j, D}^{\prime}$.

Lemma 7.5. (a) $\varphi \in L_{\frac{d^{\prime}}{2}+1, D}^{2}\left(K^{\prime}\right)$ if and only if the odd extension belongs to $L_{\frac{d^{\prime}}{2}+1}^{2}\left(\widetilde{K}^{\prime}\right) . \quad(\mathrm{b}) \varphi \in L_{\frac{d^{\prime}}{2}+1, N}^{2}\left(K^{\prime}\right)$ if and only if the even extension belongs to $L_{\frac{d^{\prime}}{2}+1}^{2}\left(\widetilde{K}^{\prime}\right)$. (c) $\varphi \in L_{\frac{d^{\prime}}{2}+1}^{2}\left(K^{\prime}\right)$ if and only if it has an extension in $L_{\frac{d^{\prime}}{2}+1}^{2}\left(\widetilde{K}^{\prime}\right)$.

Proof. The first two statements follow immediately from the definitions. It is also clear that the restriction of function in $L_{\frac{d^{\prime}}{2}+1}^{2}\left(\widetilde{K}^{\prime}\right)$ lies in $L_{\frac{d^{\prime}}{2}+1}^{2}\left(K^{\prime}\right)$. It remains to show that harmonic functions can be extended. But this is easy. We simply have to attach a biharmonic spline at each boundary point. Choose $m$ large enough so that every cell of level $m$ contains at most one boundary point. Let $h$ be the harmonic function we wish to extend. Record the boundary data $\left(h\left(q_{i}^{\prime}\right), \partial_{n} h\left(q_{i}^{\prime}\right)\right)$ at the boundary points. Let $b\left(x^{\prime}\right)$ denote the biharmonic spline determined by the data $\left(b\left(x^{\prime}\right), \partial_{n} b\left(x^{\prime}\right)\right)=(0,0)$ if $x^{\prime} \in V_{m}$ but $x^{\prime} \neq q_{i}^{\prime}$ for any $i$, and $\left(b\left(q_{i}^{\prime}\right), \partial_{n} b\left(q_{i}^{\prime}\right)\right)=$ $\left(h\left(q_{i}^{\prime}\right),-\partial_{n} h\left(q_{i}^{\prime}\right)\right)$. The extension $\widetilde{h}$ of $h$ is then just the even reflection of $b$. Note that the matching conditions imply that $\widetilde{h} \in \operatorname{dom}_{L^{2}} \widetilde{\Delta}$ with

$$
\widetilde{\Delta} \widetilde{h}= \begin{cases}0 & \text { on } K^{\prime} \\ \widetilde{\Delta} b & \text { on } \widetilde{K}^{\prime} \backslash K^{\prime}\end{cases}
$$

Since $\operatorname{dom}_{L^{2}} \widetilde{\Delta}=L_{d^{\prime}+1}^{2}(\widetilde{K}) \subseteq L_{\frac{d^{\prime}}{2}+1}^{2}(\widetilde{K})$ the result follows.

Theorem 7.6. If $u \in \operatorname{dom}_{L^{2}} \widetilde{\Delta}$, then $\operatorname{Tr}_{\partial K}(u)$ belongs to $L_{\frac{d^{\prime}}{2}+1}^{2}(\partial K)$. Conversely, there exists a bounded linear extension operator

$$
E: L_{\frac{d^{\prime}}{2}+1}^{2}(\partial K) \rightarrow \operatorname{dom}_{L^{2}} \widetilde{\Delta} .
$$

Proof. Let $u \in \operatorname{dom}_{L^{2}} \widetilde{\Delta}$. Since $\operatorname{dom}_{L^{2}} \widetilde{\Delta}$ consists of continuous functions, $\operatorname{Tr}_{\partial K}(u)$ is also continuous. Then $u \in L_{\frac{d^{\prime}}{2}+1}^{2}(\partial K)$ by Theorem 7.1, Definition 7.4 and Lemma 7.5 (c). 
Conversely, let $\varphi \in L_{\frac{d^{\prime}}{2}+1}^{2}(\partial K)$. Then there is piecewise harmonic function on $\partial K$ taking the same values as $\varphi$ on the distinguished points $\left(q_{i}^{\prime}, q_{j}^{\prime}\right)$. This function extends naturally to a pluriharmonic function on $K$, which is just a linear combination of products of harmonic functions in each factor. If we use the extension described in the proof of Lemma 7.5 (c) in each variable, we obtain an extension in $\operatorname{dom}_{L^{2}} \widetilde{\Delta}$. Thus we may assume, without loss of generality, that $\varphi\left(q_{i}^{\prime}, q_{j}^{\prime}\right)=0$ for all $i$ and $j$. Then we can write $\varphi$ as a finite sum of functions in $L_{\frac{d^{\prime}}{2}+1}^{2}(\partial K)$, each vanishing on all but one face, say $K^{\prime} \times q_{i}^{\prime}$.

So let $\varphi \in L_{\frac{d^{\prime}}{2}+1}^{2}\left(K^{\prime} \times q_{i}^{\prime}\right)$, vanishing on the boundary. Then we can again write $\varphi$ in the Dirichlet expansion (7.6), where now

$$
\sum_{j=1}^{\infty} a_{j}(\varphi)^{2}\left(\lambda_{j, D}^{\prime}\right)^{\frac{d^{\prime}+2}{d^{\prime}+1}}=\|\varphi\|_{L_{\frac{d^{\prime}}{2}+1}^{2}}^{2}<\infty
$$

By taking odd extensions this gives us an expansion on $\widetilde{K}^{\prime} \times q_{i}^{\prime}$. Once again we seek an extension of the form (7.8), but for a different choice of functions $\psi_{j}$ on $\widetilde{K}^{\prime}$. In this case we will use biharmonic splines, so that

$$
\widetilde{\Delta} E \varphi\left(x^{\prime}, x^{\prime \prime}\right)=\sum_{j=1}^{\infty} a_{j}(\varphi)\left(-\lambda_{j, D}^{\prime} u_{j, D}^{\prime}\left(x^{\prime}\right) \psi_{j}\left(x^{\prime \prime}\right)+u_{j, D}^{\prime}\left(x^{\prime}\right) \Delta^{\prime} \psi_{j}\left(x^{\prime \prime}\right)\right) .
$$

Since this expression is already orthogonal with respect to $d \mu^{\prime}\left(x^{\prime}\right)$, we see that $E \varphi \in \operatorname{dom}_{L^{2}} \widetilde{\Delta}$ if and only if

$$
\sum a_{j}(\varphi)^{2}\left(\left(\lambda_{j, D}^{\prime}\right)^{2}\left\|\psi_{j}\right\|_{2}^{2}+\left\|\widetilde{\Delta}^{\prime} \psi_{j}\right\|_{2}^{2}\right)<\infty
$$

Thus, instead of (7.9) and (7.10), we need

$$
\left\|\widetilde{\Delta}^{\prime} \psi_{j}\right\|_{2}^{2} \leq c\left(\lambda_{j, D}^{\prime}\right)^{\frac{d^{\prime}+2}{d^{\prime}+1}}
$$

and (7.10).

We take $\psi_{j}$ to be piecewise biharmonic splines of level $m$ with $\left(\psi_{j}\left(q_{i}^{\prime}\right), \partial_{n} \psi_{j}\left(q_{i}^{\prime}\right)\right)=$ $(1,0)$ and $\left(\psi_{j}\left(x^{\prime}\right), \partial_{n} \psi_{j}\left(x^{\prime}\right)\right)=(0,0)$ for all $x^{\prime} \in \tilde{V}_{m}^{\prime} \backslash\left\{q_{i}^{\prime}\right\}$. This makes $\psi_{j}$ even on $\widetilde{K}^{\prime}$. We then have $\left\|\psi_{j}\right\|_{2}^{2}=c_{1}\left(\mu_{i}^{\prime}\right)^{m}$ and $\left\|\widetilde{\Delta}^{\prime} \psi_{j}\right\|_{2}^{2}=c_{2}\left(r_{i}^{\prime}\right)^{-2 m}\left(\mu_{i}^{\prime}\right)^{-m}$. Choosing $m$ as in the proof of Theorem 7.3 yields (7.10) and (7.15).

Theorem 7.7. Let $u \in \operatorname{dom}_{L^{2}} \Delta$. Then $u$ extends to a function in $\operatorname{dom}_{L^{2}} \widetilde{\Delta}$ if and only if $\operatorname{Tr}_{\partial K}(u) \in L_{\frac{d^{\prime}}{2}+1}^{2}(\partial K)$.

Proof. If $u$ extends to a function in $\operatorname{dom}_{L^{2}} \widetilde{\Delta}$, then $\operatorname{Tr}_{\partial K}(u) \in L_{\frac{d^{\prime}}{2}+1}^{2}(\partial K)$ by Theorem 7.6. Conversely, suppose $\operatorname{Tr}_{\partial K}(u) \in L_{\frac{d^{\prime}}{2}+1}^{2}(\partial K)$. Then $E \varphi \in \operatorname{dom}_{L^{2}} \widetilde{\Delta}$ for $\varphi=\operatorname{Tr}_{\partial K}(u)$ by Theorem 7.6. Consider $u-E \varphi$ on $K$. Then $\operatorname{Tr}_{\partial K}(u-E \varphi)=$

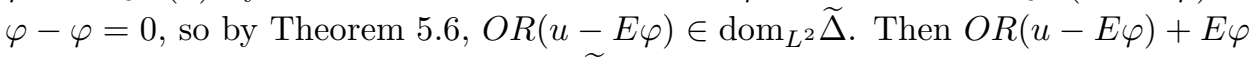
is the desired extension of $u$ in $\operatorname{dom}_{L^{2}} \widetilde{\Delta}$.

Next we consider traces of normal derivatives on $\partial K$. For this we will need to assume the following estimate on normal derivatives of the heat kernel:

$$
\partial_{n} \partial_{n} \widetilde{p}_{t}^{\prime}\left(q_{i}^{\prime}, q_{i}^{\prime}\right) \leq c t^{-\left(\frac{d^{\prime}+2}{d^{\prime}+1}\right)}
$$


(i.e., the normal derivative with respect to both variables). We could just as well use the Dirichlet heat kernel since the Neumann eigenfunctions have vanishing normal derivatives. Numerical evidence for (7.16) in the case of SG has been found by Nitsan Ben-Gal, and may be found at the website www.math.cornell.edu/ Bengal/ and in [BSSY]. The evidence suggests a more precise conjecture.

Theorem 7.8. Assume (7.16). Let $u \in \operatorname{dom}_{L^{2}} \widetilde{\Delta}$. Then $\partial_{n} u\left(x^{\prime}, q_{i}^{\prime}\right) \in L_{\frac{d^{\prime}}{2}}^{2}\left(K^{\prime}\right)$ and $\partial_{n} u\left(q_{i}^{\prime}, x^{\prime \prime}\right) \in L_{\frac{d^{\prime}}{2}}^{2}\left(K^{\prime}\right)$ for each $i$.

Remark. By abuse of notation we write $\left.\partial_{n} u\right|_{\partial K} \in L_{\frac{d^{\prime}}{2}}^{2}(\partial K)$. Since $d^{\prime} / 2$ is the borderline case, we should really require a compatibility condition on the separate $L_{\frac{d^{\prime}}{2}}^{2}\left(K^{\prime}\right)$ pieces to define the space $L_{\frac{d^{\prime}}{2}}^{2}(\partial K)$. However, in this situation the normal derivatives come from different directions, so there is no compatibility condition.

Proof. We follow the outine of the proof of Theorem 7.1. This time we need the estimate

$$
\sum_{j}\left(\widetilde{\lambda}_{j}^{\prime}+\widetilde{\lambda}_{k}^{\prime}\right)^{-2}\left|\partial_{n} \widetilde{u}_{j}^{\prime}\left(q_{i}^{\prime}\right)\right|^{2} \leq c\left(\widetilde{\lambda}_{k}^{\prime}\right)^{-\frac{d^{\prime}}{d^{\prime}+1}} .
$$

Now we observe that

$$
\sum_{j} e^{-\widetilde{\lambda}_{j}^{\prime} t}\left|\partial_{n} \widetilde{u}_{j}^{\prime}\left(q_{i}^{\prime}\right)\right|^{2}=\partial_{n} \partial_{n} \widetilde{p}_{t}^{\prime}\left(q_{i}^{\prime}, q_{i}^{\prime}\right)
$$

and so

$$
\sum_{j}\left({\widetilde{\lambda_{j}}}_{j}+\widetilde{\lambda}_{k}^{\prime}\right)^{-2}\left|\partial_{n} \widetilde{u}_{j}^{\prime}\left(q_{i}^{\prime}\right)\right|^{2}=\int_{0}^{\infty} e^{-\widetilde{\lambda}_{k}^{\prime} t} \partial_{n} \partial_{n} \widetilde{p}_{i}^{\prime}\left(q_{i}^{\prime}, q_{i}^{\prime}\right) t d t
$$

If we substitute the estimate (7.16) we obtain (7.17).

Theorem 7.9. Suppose $d^{\prime}>1$. There exists a bounded linear operator $E_{N}$ : $L_{\frac{d^{\prime}}{2}}^{2}(\partial K) \rightarrow \operatorname{dom}_{L^{2}} \widetilde{\Delta}$ such that $\left.\partial_{n} E_{N} \varphi\right|_{\partial K}=\varphi$.

Proof. We use the same idea as in the proof of Theorem 7.3, but this time we expand $\varphi$ on each piece of the boundary in a series of Neumann eigenfunctions. We may assume that $\varphi$ vanishes except on $K^{\prime} \times q_{i}^{\prime}$, where

$$
\varphi\left(x^{\prime}, q_{i}^{\prime}\right)=\sum_{j=0}^{\infty} a_{j}(\varphi) u_{j, N}^{\prime}\left(x^{\prime}\right)
$$

and

$$
\sum_{j=0}^{\infty} a_{j}(\varphi)^{2}\left(\lambda_{j, N}^{\prime}\right)^{\frac{d^{\prime}}{d^{\prime}+1}}=\|\varphi\|_{L_{\frac{d^{\prime}}{2}}^{2}(\partial K)}^{2}<\infty .
$$

We take our extension operator of the form

$$
E_{N} \varphi\left(x^{\prime}, x^{\prime \prime}\right)=\sum_{j=0}^{\infty} a_{j}(\varphi) u_{j, N}^{\prime}\left(x^{\prime}\right) \psi_{j}\left(x^{\prime \prime}\right)
$$

for the appropriate choice of $\psi_{j}$. This time we want $\partial_{n} \psi_{j}\left(q_{\ell}^{\prime}\right)=\delta_{j \ell}$ and the sum in (7.20) to be finite for $x^{\prime \prime} \neq q_{i}^{\prime}$, so as to have $\left.\partial_{n} E_{N} \varphi\right|_{\partial K}=\varphi$. As in the proof of Theorem 7.6 we will take $\psi_{j}$ to be a piecewise biharmonic spline of level $m$ with $\left(\psi_{j}\left(q_{i}^{\prime}\right), \partial_{n} \psi_{j}\left(q_{i}^{\prime}\right)\right)=(0,1)$ and $\left(\psi_{j}\left(x^{\prime}\right), \partial_{n} \psi_{j}\left(x^{\prime}\right)\right)=(0,0)$ for all $x^{\prime} \in \tilde{V}_{m}^{\prime} \backslash\left\{q_{i}^{\prime}\right\}$. 
Note that $\psi_{j}$ extends to be odd on $\widetilde{K}^{\prime}$, while $u_{j, N}^{\prime}$ extends to be even on $\widetilde{K}^{\prime}$. To show that $E_{N}$ maps to $\operatorname{dom}_{L^{2}} \widetilde{\Delta}$ we need to bound

$$
\sum_{j=1}^{\infty} a_{j}(\varphi)^{2}\left(\left(\lambda_{j, N}^{\prime}\right)^{2}\left\|\psi_{j}\right\|_{2}^{2}+\left\|\widetilde{\Delta}^{\prime} \psi_{j}\right\|_{2}^{2}\right)
$$

by a multiple of (7.19). In other words we need

$$
\left\|\psi_{j}\right\|_{2}^{2} \leq c\left(\lambda_{j, N}^{\prime}\right)^{-\left(\frac{d^{\prime}+2}{d^{\prime}+1}\right)}
$$

and

$$
\left\|\widetilde{\Delta}^{\prime} \psi_{j}\right\|_{2}^{2} \leq c\left(\lambda_{j, N}^{\prime}\right)^{\frac{d^{\prime}}{d^{\prime}+1}} .
$$

In order to have $\partial_{n} \psi_{j}\left(q_{i}^{\prime}\right)=1$ we need to have the values of $\psi_{j}$ on $\left(F_{j}^{\prime}\right)^{m} K^{\prime}$ to be of size $\left(r_{i}^{\prime}\right)^{m}$, so $\left\|\psi_{j}\right\|_{2}^{2} \approx\left(\mu_{i}^{\prime}\right)^{m}\left(r_{i}^{\prime}\right)^{2 m}$ and $\left\|\widetilde{\Delta}^{\prime} \psi_{j}\right\|_{2}^{2} \approx\left(\mu_{i}^{\prime}\right)^{-m}$. Once again the choice of $m$ in the proof of Theorem 7.3 (with $\lambda_{j, N}^{\prime}$ replacing $\lambda_{j, D}^{\prime}$ ) gives (7.21) and (7.22).

Conjecture 7.10. Suppose $d^{\prime}>1$. Let $u \in d_{\widetilde{A}} m_{L^{2}} \Delta$. Then $u$ extends to a function in dom $_{L^{2}} \widetilde{\Delta}$ if and only if $\left.\partial_{n} u\right|_{\partial K} \in L_{\frac{d^{\prime}}{2}}^{2}(\partial K)$.

The only difficulty in adapting the proof of Theorem 7.7 is to supply the missing converse to Theorem 5.7.

\section{REPRESENTATION OF HARMONIC FUNCTIONS}

We have seen that the maximum principle implies that a harmonic function $h$ on $K$ is determined by its boundary values $\left.h\right|_{\partial K}=\varphi$ by integration of $\varphi$ with respect to a "harmonic measure" on the boundary. We expect to be able to write this more precisely as a Poisson integral formula

$$
h(x)=\int_{\partial K} P(x, y) \varphi(y) d \nu(y)
$$

with a Poisson kernel $P$ defined on $K \times \partial K$ by

$$
P(x, y)=-\partial_{n}(y) G_{D}(x, y) .
$$

Theorem 8.1. Let $h$ be a harmonic function of finite energy on $K$, with boundary values $\varphi \in L_{1 / 2}^{2}(\partial K)$. Then (8.1) holds for all $x$ in the interior of $K$, where $P$ is given by (8.2).

Proof. We prove the result first for $\varphi \in L_{\frac{d^{\prime}}{2}+1}^{2}(\partial K)$, so $h \in L_{d^{\prime}+1}^{2}(K)$. Since $L_{\frac{d^{\prime}}{2}+1}^{2}(\partial K)$ is dense in $L_{\frac{1}{2}}^{2}(\partial K)$, the result follows by continuity.

There is a very simple formal proof of (8.1), namely

$$
\begin{aligned}
h(x) & =\int_{K}\left(\Delta_{y} h(y) G_{D}(x, y)-h(y) \Delta_{y} G_{D}(x, y)\right) d \mu(y) \\
& =\int_{\partial K}\left(\partial_{n}(y) h(y) G_{D}(x, y)-h(y) \partial_{n}(y) G_{D}(x, y)\right) d \nu(y)
\end{aligned}
$$

by the Gauss-Green formula, with the first integrals vanishing because $\Delta_{y} h(y)=0$ and $G_{D}(x, y)=0$ for $y \in \partial K$. Of course the Gauss-Green formula has not been established in this singular setting where $\Delta_{y} G_{D}(x, y)=\delta(x, y)$, so we give a more roundabout argument. Let $v \in L_{d^{\prime}+1}^{2}(K)$ with $\left.v\right|_{\partial K}=\varphi$. Such $v$ exists by Theorem 
7.6, and the proof shows that for any fixed $x_{0}$ in the interior of $K$ we can choose $v$ so that $x_{0}$ is not in the support of $v$. Therefore, $\Delta(h-v)=-\Delta v$ and $\left.(h-v)\right|_{\partial K}=0$, so

In particular

$$
h(x)-v(x)=\int_{K} G_{D}(x, y) \Delta_{y} v(y) d \mu(y) .
$$

$$
h\left(x_{0}\right)=\int_{D} G_{D}\left(x_{0}, y\right) \Delta_{y} v(y) d \mu(y)
$$

and $G_{D}\left(x_{0}, \cdot\right)$ is a harmonic function on the support of $\Delta_{y} v(y)$. Now there is no singularity so Gauss-Green yields

$$
h\left(x_{0}\right)=\int_{\partial K} G_{D}\left(x_{0}, y\right) \partial_{n}(y) v(y) d \nu(y)-\int_{\partial K} \varphi(y) \partial_{n}(y) G_{D}\left(x_{0}, y\right) d \nu(y)
$$

and the first integral vanishes since $G_{D}\left(x_{0}, y\right)=0$ for $y \in \partial K$.

For the next result we need to assume the following estimate holds for the normal derivative of the heat kernel:

$$
\left|\partial_{n} p_{t, D}^{\prime}\left(x^{\prime \prime}, q_{i}^{\prime}\right)\right| \leq c t^{-\left(\frac{d^{\prime}+2}{d^{\prime}+1}\right)} d^{\prime}\left(x^{\prime \prime}, q_{i}^{\prime}\right) \exp \left(-c\left(\frac{d^{\prime}\left(x^{\prime \prime}, q_{i}^{\prime}\right)^{d^{\prime}+1}}{t}\right)^{J}\right) .
$$

Theorem 8.2. Assume (8.3) holds. The Poisson kernel $P(x, y)$ is continuous for $x$ in the interior of $K$, and satisfies the following estimate for $y$ in the piece $K^{\prime} \times q_{i}^{\prime}$ of the boundary:

$$
\left|P\left(\left(x^{\prime}, x^{\prime \prime}\right),\left(y^{\prime}, q_{i}^{\prime}\right)\right)\right| \leq c \frac{d^{\prime}\left(x^{\prime \prime}, q_{i}^{\prime}\right)}{d(x, y)^{d^{\prime}+1}},
$$

and similar estimates on the other pieces.

Proof. We use (8.2) together with

$$
G_{D}(x, y)=\int_{0}^{\infty} p_{t, D}(x, y) d t
$$

to obtain

$$
P\left(\left(x^{\prime}, x^{\prime \prime}\right),\left(y^{\prime}, q_{i}^{\prime}\right)\right)=-\int_{0}^{\infty} p_{t, D}^{\prime}\left(x^{\prime}, y^{\prime}\right) \partial_{n}\left(y^{\prime \prime}\right) p_{t, D}^{\prime}\left(x^{\prime \prime}, q_{i}^{\prime}\right) d t .
$$

Substitute (8.3) into (8.5) to obtain (8.4). The continuity of $P(x, y)$ follows easily from these estimates and the continuity of the heat kernel.

In particular, it follows that harmonic functions are continuous away from the boundary.

There are analogous results for the solution of the Neumann problem. If $h \in$ $L_{d^{\prime}+1}^{2}(K)$ is harmonic, then

$$
\psi=\left.\partial_{n} h\right|_{\partial K} \in L_{\frac{d^{\prime}}{2}}^{2}(\partial K) \text { and } \int_{\partial K} \psi d \nu=0 .
$$

We can recover $h$, up to a constant, by

$$
h(x)=c+\int_{\partial K} G_{N}(x, y) \psi(y) d \nu(y) .
$$

The proof of (8.6) is similar to the proof of Theorem 8.1, so we omit the details. Using estimates as in Corollary 6.3 we obtain

$$
\left|G_{N}(x, y)\right| \leq c d(x, y)^{1-d^{\prime}}
$$


provided $d^{\prime}>1$ (in the case of the square there are logarithmic terms).

Of course our expectation is that the representation (8.1) should yield a harmonic function on the interior of $K$ for more general boundary values, at least $\varphi \in L^{2}\left(K^{\prime}\right)$ or $\varphi \in L^{1}\left(K^{\prime}\right)$, not to mention negative order Sobolev spaces defined by duality. This would follow easily if we could show that uniform limits of harmonic functions are harmonic. We also note that (8.1) is a kind of mean value property for harmonic functions.

Instead of using (8.1) for all $x$ in the interior of $K$, we may also consider just restricting it to the boundary of cells of order 1 (that is, just the portion not already in $\partial K$ ). In this way we obtain a "harmonic extension algorithm" that enables us to go from the boundary values of $h$ on $\partial K$ to the boundary values of $h$ on $\partial F_{w} K$ for all $|w|=1$. Then by iteration ( $h \circ F_{w}$ is also harmonic) we could obtain the values of $h$ on $\partial F_{w} K$ for all words $w$. This would be a way of generating harmonic functions that exploits the self-similar structure of $K$. However, it should be kept in mind that such a procedure in the case of the square has never been explicitly understood or utilized.

The following formula for the energy of a harmonic function in terms of its boundary values is known in the case of the standard Laplacian on domains in Euclidean space (see $[\mathrm{SB}]$ or $[\mathrm{BrP}]$ ).

Theorem 8.3. Let $h$ be harmonic in $K$ with finite energy having an extension in $\operatorname{dom}_{L^{2}} \widetilde{\Delta}$, and let $\varphi=\left.h\right|_{\partial K}$. Then

$$
\mathcal{E}(h, h)=\frac{1}{2} \int_{\partial K} \int_{\partial K}|\varphi(y)-\varphi(z)|^{2} \partial_{n} \partial_{n} G_{D}(y, z) d \nu(y) d \nu(z) .
$$

Proof. By Theorem 5.4 (b) we have

$$
\mathcal{E}(h, h)=\int_{\partial K} \varphi(y) \partial_{n} h(y) d \nu(y) .
$$

We use (8.1) to compute $\partial_{n} h(y)$. Note that we can write

$$
h(y)-h(x)=\int_{\partial K} P(x, z)(\varphi(y)-\varphi(z)) d \nu(z)
$$

because $\int_{\partial K} P(x, z) d \nu(z)=1$. Also for $y \neq z$ we have $P(y, z)=0$, so that

$$
h(y)-h(x)=\int_{\partial K}(P(y, z)-P(x, z))(\varphi(z)-\varphi(y)) d \nu(z) .
$$

From the definition of the normal derivative we obtain

$$
\partial_{n} h(y)=\int_{\partial K}\left(\partial_{n}(y) P(y, z)\right)(\varphi(z)-\varphi(y)) d \nu(z) .
$$

Now substitute (8.10) in (8.9) and use (8.2) to obtain

$$
\mathcal{E}(h, h)=\int_{\partial K} \int_{\partial K} \varphi(y)(\varphi(y)-\varphi(z)) \partial_{n} \partial_{n} G_{D}(y, z) d \nu(y) d \nu(z) .
$$

By symmetry this is the same as (8.8).

Next we give estimates for the kernel $\partial_{n} \partial_{n} G_{D}(y, z)$ in (8.8). For this we need the assumption

$$
\partial_{n} \partial_{n} p_{t, D}\left(q_{i}^{\prime}, q_{\ell}^{\prime}\right) \leq c_{1} e^{-c_{2} t} \text { for } i \neq \ell .
$$


Theorem 8.4. Assume (7.16), (8.3), and (8.11). (a) For $y=\left(y^{\prime}, q_{i}^{\prime}\right)$ and $z=$ $\left(z^{\prime}, q_{i}^{\prime}\right)$ in the same piece of $\partial K$ we have

$$
\partial_{n} \partial_{n} G_{D}(y, z) \leq c d^{\prime}\left(y^{\prime}, z^{\prime}\right)^{-\left(d^{\prime}+1\right)} .
$$

(b) For $y=\left(y^{\prime}, q_{i}^{\prime}\right)$ and $z=\left(q_{\ell}^{\prime}, z^{\prime \prime}\right)$ in adjacent pieces of $\partial K$ we have

$$
\partial_{n} \partial_{n} G_{D}(y, z) \leq \frac{c d^{\prime}\left(y^{\prime}, q_{\ell}^{\prime}\right) d^{\prime}\left(z^{\prime \prime}, q_{i}^{\prime}\right)}{d(y, z)^{d^{\prime}+3}} .
$$

(c) For $y=\left(y^{\prime}, q_{i}^{\prime}\right)$ and $z=\left(z^{\prime}, q_{\ell}^{\prime}\right)$ for $i \neq \ell$ in disjoint pieces of $\partial K$ we have

$$
\partial_{n} \partial_{n} G_{D}(y, z) \leq c .
$$

In all three cases we have

$$
\partial_{n} \partial_{n} G_{D}(y, z) \leq c d(y, z)^{-\left(d^{\prime}+1\right)},
$$

although (8.13) is a more precise estimate than (8.15) in case (b).

Proof. In all cases we use

$$
\partial_{n} \partial_{n} G_{D}(y, z)=\int_{0}^{\infty} \partial_{n} \partial_{n} p_{t, D}(y, z) d t .
$$

In (a) we have

$$
\partial_{n} \partial_{n} p_{t, D}(y, z)=p_{t, D}^{\prime}\left(y^{\prime}, z^{\prime}\right) \partial_{n} \partial_{n} p_{t, D}^{\prime}\left(q_{i}^{\prime}, q_{i}^{\prime}\right) .
$$

Using (6.8) and (7.16) we obtain

$$
\partial_{n} \partial_{n} p_{t, D}(y, z) \leq c t^{-2} \exp \left(-c\left(\frac{d^{\prime}\left(y^{\prime}, z^{\prime}\right)^{d^{\prime}+1}}{t}\right)^{J}\right) .
$$

Substitute this into (8.16) to obtain (8.12). In (b) we have

$$
\partial_{n} \partial_{n} p_{t, D}(y, z)=\partial_{n} p_{t, D}^{\prime}\left(y^{\prime}, q_{\ell}^{\prime}\right) \partial_{n} p_{t, D}^{\prime}\left(q_{i}^{\prime}, z^{\prime \prime}\right)
$$

Using (8.3) we obtain

$$
\partial_{n} \partial_{n} p_{t, D}(y, z) \leq c t^{-\left(\frac{2 d^{\prime}+4}{d^{\prime}+1}\right)} d^{\prime}\left(y^{\prime}, q_{\ell}^{\prime}\right) d^{\prime}\left(z^{\prime \prime}, q_{\ell}^{\prime}\right) \exp \left(-c\left(\frac{d(y, z)^{d^{\prime}+1}}{t}\right)^{J}\right) .
$$

Substitute this into (8.16) to obtain (8.13). In (c) we have

$$
\partial_{n} \partial_{n} p_{t, D}(y, z)=p_{t, D}^{\prime}\left(y^{\prime}, z^{\prime}\right) \partial_{n} \partial_{n} p_{t, D}^{\prime}\left(q_{i}^{\prime}, q_{\ell}^{\prime}\right)
$$

for $i \neq \ell$. Using (6.8), (8.11) and (8.16) we obtain (8.14).

Estimates analogous to (8.15) are given in $\mathrm{BrP}$ for Euclidean domains. In the case of the square we can see that (8.13) is sharp. It is easier to do the computation for the quarter complex plane $0 \leq \arg z \leq \pi / 2$ and conformally map it by $z^{2}$ onto the half-plane. We find that in (a) with $y$ and $z$ on the positive real axis

$$
\partial_{n} \partial_{n} G_{D}(y, z)=\frac{c y z}{(y+z)^{2}(y-z)^{2}} .
$$

In (b), with $y>0$ and $z=i w$ with $w \geq 0$

$$
\partial_{n} \partial_{n}(y, z)=\frac{c y w}{\left(y^{2}+w^{2}\right)^{2}} .
$$

There is another approach to the representation of harmonic functions that is well known in the case of the square. We assume first that $\varphi \in L_{\frac{d^{\prime}}{2}+1}^{2}(\partial K)$. We can easily construct a function in $\mathrm{PH}$ that matches the values of $\varphi$ on $V_{0}$, so we may assume without loss of generality that $\varphi$ vanishes on $V_{0}$. Then on each piece 
$K^{\prime} \times q_{i}^{\prime \prime}$ of the boundary (and similarly for $q_{i}^{\prime} \times K^{\prime \prime}$ ) we expand $\varphi$ in a Dirichlet eigenfunction expansion,

$$
\varphi\left(x^{\prime}, q_{i}^{\prime \prime}\right)=\sum_{j=1}^{\infty} a_{j} u_{j, D}^{\prime}\left(x^{\prime}\right)
$$

with

$$
\sum_{j=1}^{\infty}\left(\lambda_{j, D}^{\prime}\right)^{\frac{d^{\prime}+2}{d^{\prime}+1}}\left|a_{j}\right|^{2}<\infty
$$

We then create the function

$$
\sum_{j=1}^{\infty} a_{j} u_{j, D}^{\prime}\left(x^{\prime}\right) \psi_{j}\left(x^{\prime \prime}\right)
$$

where $\psi_{j}\left(x^{\prime \prime}\right)$ satisfies

$$
\Delta^{\prime \prime} \psi_{j}=\lambda_{j, D}^{\prime} \psi_{j} \text { on } K^{\prime \prime}
$$

with boundary conditions

$$
\psi_{j}\left(q_{\ell}^{\prime \prime}\right)=\delta(\ell, i)
$$

Note that the eigenvalue equation (8.20) has the wrong sign, so (8.19) is the analog of the expansion

$$
\sum_{j=1}^{\infty} a_{j} \sin \pi j x^{\prime} \frac{\sinh \pi j x^{\prime \prime}}{\sinh \pi j}
$$

in the case of the square. At least formally (8.19) is a harmonic function in $K$ whose boundary values vanish on every piece of $\partial K$ except $K^{\prime} \times q_{i}^{\prime \prime}$, and on that piece it equals $\varphi$ by (8.17). Thus by adding together such contributions from each piece of the boundary we obtain the harmonic function with boundary values $\varphi$.

It remains to examine the convergence of (8.19). We claim that as long as $x^{\prime \prime}$ lies in the interior of $K^{\prime \prime}$, the factor $\psi_{j}\left(x^{\prime \prime}\right)$ will decay exponentially. (This is well known on Riemannian manifolds; see $\left[\mathrm{Be}\right.$.) Suppose $d^{\prime}\left(x^{\prime \prime}, q_{i}^{\prime}\right) \geq \varepsilon$. Reasoning as in the proof of Theorem 8.1 we can write

$$
\psi_{j}\left(x^{\prime \prime}\right)=\int_{0}^{\infty} e^{-t \lambda} \partial_{n} p_{t, D}^{\prime}\left(q_{i}^{\prime}, x^{\prime \prime}\right) d t,
$$

where we put $\lambda=\lambda_{j, D}^{\prime}$ for simplicity of notation. Assuming (8.3) we obtain

$$
\left|\psi_{j}\left(x^{\prime \prime}\right)\right| \leq c \int_{0}^{\infty} e^{-t \lambda} t^{-\left(\frac{d^{\prime}+2}{d^{\prime}+1}\right)} \exp \left(-c\left(\frac{\varepsilon^{d^{\prime}+1}}{t}\right)^{J}\right) d t
$$

Splitting the integral at $t=\varepsilon^{\left(\frac{J}{J+1}\right)\left(d^{\prime}+1\right)} \lambda^{-1 /(J+1)}$ we obtain the estimate

$$
\left|\psi_{j}\left(x^{\prime \prime}\right)\right| \leq c \exp \left(-c \varepsilon^{\left(\frac{J}{J+1}\right)\left(d^{\prime}+1\right)}\left(\lambda_{j, D}^{\prime}\right)^{\left(\frac{J}{J+1}\right)}\right) .
$$

This is sufficient to make (8.19) converge, even without (8.18) (as long as $\varphi \in L^{2}$ ), and to justify applying the operators $\Delta^{\prime}$ and $\Delta^{\prime \prime}$ term by term. In particular, the representation is valid as long as the harmonic function, and hence its boundary values, is assumed continuous. 
The representation (8.19) is very useful if the boundary values $\varphi$ are supported on a single piece of the boundary, because the individual terms are orthogonal with respect to both $L^{2}$ and energy. In that case the total energy of (8.19) is

$$
\sum_{j=1}^{\infty}\left|a_{j}\right|^{2}\left(\lambda_{j, D}^{\prime}\left\|\psi_{j}\right\|_{2}^{2}+\mathcal{E}^{\prime \prime}\left(\psi_{j}, \psi_{j}\right)\right)
$$

It is less useful when we have to add contributions from more than one piece, since it is difficult to understand the interactions between them.

In Section 11 we will use the expansion (8.19) and the estimate (8.25), but we will also need a local version. Suppose $C=C^{\prime} \times C^{\prime \prime}$, where $C^{\prime}$ and $C^{\prime \prime}$ are simple subsets of $K^{\prime}$ and $K^{\prime \prime}$. Then we have complete orthonormal bases of Dirichlet eigenfunctions on $C^{\prime}$ and $C^{\prime \prime}$, and corresponding Dirichlet heat kernels that satisfy estimates (6.8). Correspond to each piece $C^{\prime} \times\left\{y^{\prime \prime}\right\}$ of $\partial C$ (here $y^{\prime \prime}$ denotes a point in the finite set $\partial C^{\prime \prime}$ ) we have an expansion analogous to (8.17) for the boundary values and hence (8.19) for a harmonic function of finite energy in $C$. Now $\psi_{j}\left(x^{\prime \prime}\right)$ satisfies (8.20) on $C^{\prime \prime}$ and boundary conditions $\psi_{j}\left(y^{\prime \prime}\right)=1$ and $\psi_{j}\left(z^{\prime \prime}\right)=0$ for $z^{\prime} \neq y^{\prime \prime}$ in $\partial C^{\prime \prime}$. Assuming the analog of (8.3) for the local Dirichlet heat kernels, we obtain the estimate $(8.25)$.

\section{Pointwise expressions}

We would like to have pointwise expressions for energy and Laplacian in terms of the values of the function on the discrete sets $V_{m}=V_{m}^{\prime} \times V_{m}^{\prime \prime}$. Although this cannot be the case in general, it is easy to accomplish for some natural dense subspaces of functions. Indeed we can create a graph $\Gamma_{m}$ with vertices $V_{m}$ and edge relation $\left(x^{\prime}, y^{\prime \prime}\right) \sim_{m}\left(y^{\prime}, y^{\prime \prime}\right)$ if either $x^{\prime \prime}=y^{\prime \prime}$ and $x^{\prime} \sim_{m} y^{\prime}$ or $x^{\prime}=y^{\prime}$ and $x^{\prime \prime} \sim_{m} y^{\prime \prime}$. If $u$ is continuous on $K$ it makes sense to restrict $u$ to $V_{m}$. Under the assumptions of Lemma 2.2 we may define a sequence of graph energies on $\Gamma_{m}$ as follows. On $\Gamma_{0}$ we define

$$
\mathcal{E}_{0}(u, u)=\sum_{x \sim 0} c_{x y}(u(x)-u(y))^{2},
$$

where the conductances $c_{x y}$ are defined by

$$
c_{x y}= \begin{cases}\frac{c_{i j}^{\prime}}{n_{i \prime}^{\prime \prime}} & \text { if } x=\left(q_{i}^{\prime}, q_{\ell}^{\prime \prime}\right) \text { and } y=\left(q_{j}^{\prime}, q_{\ell}^{\prime \prime}\right), \\ \frac{c_{i j}^{\prime \prime}}{n^{\prime}} & \text { if } x=\left(q_{\ell}^{\prime}, q_{i}^{\prime \prime}\right) \text { and } y=\left(q_{\ell}^{\prime}, q_{j}^{\prime \prime}\right) .\end{cases}
$$

On $\Gamma_{m}$ we define

$$
\mathcal{E}_{m}(u, u)=\sum_{|w|=m} \frac{1}{b^{m} r_{w^{\prime}}^{\prime} r_{w^{\prime \prime}}^{\prime \prime}} \mathcal{E}_{0}\left(u \circ F_{w}, u \circ F_{w}\right) .
$$

Note that we also may write

$$
\mathcal{E}_{m}(u, u)=\sum_{x \sim m} c_{x y}(u(x)-u(y))^{2}
$$

for certain conductances $c_{x y}$, but the exact formula for $c_{x y}$ is a bit complicated since the same edge may appear several times in (9.3). There is no claim that the sequence $\left\{\mathcal{E}_{m}(u, u)\right\}$ is monotone increasing.

The pointwise expression for energy would then be

$$
\mathcal{E}(u, u)=\lim _{m \rightarrow \infty} \mathcal{E}_{m}(u, u) .
$$


It is easy to see that (9.5) holds for $u \in \mathrm{PPH}$, or if $u$ is represented by a finite Neumann expansion (2.15).

It would be naive to hope that (9.5) would be valid for all continuous functions in dom $\mathcal{E}$, or that the existence of the limit in (9.5) for a continuous function would imply that the function belongs to $\operatorname{dom} \mathcal{E}$. In any case, it is not natural to assume the function is continuous. The next result is established only for $K=$ $\mathrm{SG}^{2}$, but presumably something similar is valid for $K=K^{\prime} \times K^{\prime}$ if $K^{\prime}$ satisfies spectral decimation. (Recall that $b^{m} r_{w^{\prime}}^{\prime} r_{w^{\prime \prime}}^{\prime \prime}=(9 / 5)^{m}$ in this case.) The idea is to simultaneously use a natural family of spectral projections $P_{m}$, and take the limit of $\mathcal{E}_{m}\left(P_{m} u, P_{m} u\right)$. We will use certain facts from OSS that are stated for Dirichlet eigenfunction expansions on SG, but the extension to the Neumann case is straightforward. Let $d_{m}=\frac{1}{2}\left(3^{m+1}+3\right)$ be the cardinality of $V_{m}^{\prime}$. Then the Neumann eigenspaces with eigenvalue $\lambda_{j}^{\prime}$ for $0 \leq j \leq d_{m}-1$ do not split multiplicities, and each eigenfunction extends from $V_{m}^{\prime}$ by spectral decimation choosing $\varepsilon_{m^{\prime}}=-1$ for $m^{\prime}>m$. The spectral projections we want to use are given by

$$
P_{m} u=\sum_{j=0}^{d_{m}-1} \sum_{k=0}^{d_{m}-1} a_{j k}(u) u_{j k} .
$$

Note that this is the analog of square summation of double Fourier series.

Theorem 9.1. Let $K=S G^{2}$. Then $u \in \operatorname{dom} \mathcal{E}$ if and only if

$$
\sup _{m} \mathcal{E}_{m}\left(P_{m} u, P_{m} u\right)<\infty
$$

in which case

$$
\mathcal{E}(u, u)=\lim _{m \rightarrow \infty} \mathcal{E}_{m}\left(P_{m} u, P_{m} u\right),
$$

and $\mathcal{E}(u, u)$ is bounded above and below by multiples of (9.7).

Proof. The key idea of the proof is that the functions $u_{j k}$ for $j, k<d_{m}$ are orthogonal with respect to $\mathcal{E}_{m}$, and there exists a universal constant $c$ such that

$$
\mathcal{E}_{m}\left(u_{j k}, u_{j k}\right) \leq c \mathcal{E}\left(u_{j k}, u_{j k}\right) .
$$

We also need the already observed fact that

$$
\mathcal{E}\left(u_{j k}, u_{j k}\right)=\lim _{m \rightarrow \infty} \mathcal{E}_{m}\left(u_{j k}, u_{j k}\right) .
$$

It is then a routine matter to complete the proof, and we omit the details.

Now $\mathcal{E}_{m}\left(u_{j k}, u_{j k}\right)$ is a sum of two terms, each being the product of a discrete approximation to $\mathcal{E}^{\prime}\left(u_{j}^{\prime}, u_{j}^{\prime}\right)$ and $\int u_{k}^{\prime} u_{k}^{\prime} d \mu^{\prime}$ (and vice versa). The approximation to $\mathcal{E}^{\prime}\left(u_{j}^{\prime}, u_{j}^{\prime}\right)$ is just $\mathcal{E}_{m}^{\prime}\left(u_{j}^{\prime}, u_{j}^{\prime}\right)$, and we know $\mathcal{E}_{m}^{\prime}\left(u_{j}^{\prime}, u_{j}^{\prime}\right) \leq \mathcal{E}^{\prime}\left(u_{j}^{\prime}, u_{j}^{\prime}\right)$, and moreover the orthogonality of $\left\{u_{j}^{\prime}\right\}$ for $j<d_{m}$ is a consequence of spectral decimation. The content of Section 2 of [OSS] is that the discrete approximation to the integral behaves similarly: the orthogonality is maintained, and moreover the discrete approximation is equal to a multiple of the integral that depends on the eigenvalue. The multiple is given by a value of a special function denoted $\widetilde{b}$ that is bounded, so that bound (about 1.63 in the graph of $\widetilde{b}$ in Figure 2.1 of [OSS] gives the constant in (9.9). Although the details of the argument in OSS are highly computational, the gist of the reason for (9.9) can be explained in a much simpler way: because we choose $\varepsilon_{m^{\prime}}=-1$ for all $m^{\prime}>m$, the extension algorithm for these eigenfunctions beyond $V_{m}$ does not differ too much from the extension algorithm for harmonic 
functions. For harmonic functions the discrete approximation to the integral is exact, so for these eigenfunctions the discrete approximation is fairly accurate.

Next we describe a discrete renormalized Laplacian $\Delta_{m}$. Let $\psi_{x}^{(m)}$ for $x \in$ $\left(V_{m}^{\prime} \backslash V_{0}^{\prime}\right) \times\left(V_{m}^{\prime \prime} \backslash V_{0}^{\prime \prime}\right)$ denote the $\mathrm{PPH}_{m}$ function satisfying $\psi_{x}^{(m)}(y)=\delta(x, y)$ for $y \in V_{m}$. We want

$$
-\mathcal{E}_{m}\left(u, \psi_{y}^{(m)}\right) \approx \int\left(\Delta_{m} u\right) \psi_{x}^{(m)} d \mu .
$$

We use the approximation

$$
\int\left(\Delta_{m} u\right) \psi_{x}^{(m)} d \mu \approx \Delta_{m}(x) \int \psi_{x}^{(m)} d \mu .
$$

Note that

$$
-\mathcal{E}_{m}\left(u, \psi_{x}^{(m)}\right)=\sum_{y \sim_{m} x} c_{x y}(u(y)-u(x))
$$

This leads to the choice

$$
\Delta_{m} u(x)=\left(\int \psi_{x}^{(m)} d \mu\right)^{-1} \sum_{y \sim_{m} x} c_{x y}(u(y)-u(x)) .
$$

Because $\psi_{x}^{(m)}$ is a product, it is easy to compute the integral. For the square

$$
\begin{gathered}
\Delta_{m} u\left(x^{\prime}, y^{\prime}\right)=4^{m}\left(u\left(x^{\prime}+\frac{1}{2^{m}}, y^{\prime}\right)+u\left(x^{\prime}-\frac{1}{2^{m}}, y^{\prime}\right)+u\left(x^{\prime}, y^{\prime}+\frac{1}{2^{m}}\right)\right. \\
\left.+u\left(x^{\prime}, y^{\prime}-\frac{1}{2^{m}}\right)-4 u\left(x^{\prime}, y^{\prime}\right)\right)
\end{gathered}
$$

is the standard second difference approximation to $\Delta u$. For SG ${ }^{2}$ we have $\int \psi_{x}^{(m)} d \mu=$ $\left(\frac{2}{3} \cdot 3^{-m}\right)^{2}$ so

The expectation is that

$$
\Delta_{m} u(x)=\frac{9}{4} 5^{m} \sum_{y \sim_{m} x}(u(y)-u(x)) .
$$

$$
\Delta u=\lim _{m \rightarrow \infty} \Delta_{m} u
$$

for a reasonable class of functions. But the true situation is likely to be quite technical.

First we should consider the case of the cover $\widetilde{K}$. It is easy to extend the discrete Laplacian to $\widetilde{\Delta}_{m}$ defined on $\widetilde{V}_{m}$ (no boundary to exclude here). It is easy to see that $\widetilde{\Delta} \widetilde{u}_{j k}=\lim _{m \rightarrow \infty} \widetilde{\Delta}_{m} \widetilde{u}_{j k}$ and so the same holds for finite linear combinations. For $u \in \operatorname{dom} \widetilde{\Delta}$, it should be possible to show $\widetilde{\Delta} u=\lim _{m \rightarrow \infty} \widetilde{\Delta}_{m}\left(\widetilde{P}_{m} u\right)$ uniformly. For $u \in \operatorname{dom}_{L^{2}} \widetilde{\Delta}$ the limit should exist in an $L^{2}$ sense. But more may be true. In the case of the 2-torus (the cover of the square) it is true that $\widetilde{\Delta} u=\lim _{m \rightarrow \infty} \widetilde{\Delta}_{m} u$ for $u \in \operatorname{dom} \widetilde{\Delta}$. The reason for this is that we have $u(x)=c+\int G(x, y) f(y) d y$ for $f$ continuous, and $\Delta_{m} G(x, y)$ for $x \in V_{m}$ behaves like an $L^{1}$ approximate identity (in fact there is no need to restrict $x$ to $V_{m}$ in this case). This observation requires rather detailed knowledge about the Green's function. It is not clear if we could obtain the same information even in the case of $\mathrm{SG}^{2}$. It is also not clear in the case of the 2 -torus whether or not we can characterize dom $\widetilde{\Delta}$ by the uniform convergence of $\widetilde{\Delta}_{m} u$. 
The situation for the square is not so nice. For one thing, we would expect to require that $u$ belong to the Sobolev space $L_{2}^{2}$ in addition to dom $\Delta$. However, although functions in $L_{2}^{2}$ extend to functions in $L_{2}^{2}$ on the 2-torus, there may not be any extension in dom $\widetilde{\Delta}$. Indeed it is easy to construct harmonic functions $u$ in $L_{2}^{2}$ of the square (essentially $z^{1+\varepsilon}$ for $\varepsilon>0$ ) such that $\Delta_{m} u$ does not converge uniformly to zero.

Further results must await future developments.

\section{Characterization of Sobolev spaces}

We discuss here a characterization of the $L^{2}$-Sobolev spaces for small orders of smoothness, in order to cast some light on some of the results of Sections 7 and 8 . We first discuss the p.c.f. case, and for simplicity we work on the double cover $\widetilde{K}^{\prime}$. By "small order" we mean specifically $s<\left(d^{\prime}+1\right) / 2$. We may then write

$$
\left\|u^{\prime}\right\|_{L_{s}^{2}\left(\widetilde{K}^{\prime}\right)}^{2}=\left\|\left(-\widetilde{\Delta}^{\prime}\right)^{\frac{s}{d^{\prime}+1}} u^{\prime}\right\|_{2}^{2}=\left\langle u^{\prime},\left(-\widetilde{\Delta}^{\prime}\right)\left(-\widetilde{\Delta}^{\prime}\right)^{\frac{2 s}{d^{\prime}+1}-1} u^{\prime}\right\rangle
$$

with $\frac{2 s}{d^{\prime}+1}-1<0$. We may also assume $u^{\prime}$ has mean value zero. We use

$$
\left(-\widetilde{\Delta}^{\prime}\right)^{\frac{2 s}{d^{\prime}+1}-1} u^{\prime}\left(x^{\prime}\right)=\int_{0}^{\infty} \int_{\widetilde{K}^{\prime}} \widetilde{p}_{t}^{\prime}\left(x^{\prime}, y^{\prime}\right) u^{\prime}\left(y^{\prime}\right) d \mu^{\prime}\left(y^{\prime}\right) t^{-\frac{2 s}{d^{\prime}+1}} d t
$$

and

to obtain

$$
\left(-\widetilde{\Delta}_{x}^{\prime}\right) \widetilde{p}_{t}^{\prime}\left(x^{\prime}, y^{\prime}\right)=-\frac{d}{d t} \widetilde{p}_{t}^{\prime}\left(x^{\prime}, y^{\prime}\right)
$$

$$
\left\|u^{\prime}\right\|_{L_{s}^{2}\left(\widetilde{K}^{\prime}\right)}^{2}=-\int_{\widetilde{K}^{\prime}} \int_{0}^{\infty} \int_{\widetilde{K}^{\prime}} u^{\prime}\left(x^{\prime}\right) \frac{d}{d t} \widetilde{p}_{t}^{\prime}\left(x^{\prime}, y^{\prime}\right) u^{\prime}\left(y^{\prime}\right) d \mu^{\prime}\left(y^{\prime}\right) t^{-\frac{2 s}{d^{\prime}+1}} d t d \mu^{\prime}\left(x^{\prime}\right) .
$$

However, since

$$
\int_{\widetilde{K}^{\prime}} \widetilde{p}_{t}^{\prime}\left(x^{\prime}, y^{\prime}\right) d \mu^{\prime}\left(y^{\prime}\right)=1
$$

we have

$$
\int_{\widetilde{K}^{\prime}} \frac{d}{d t} \widetilde{p}_{t}^{\prime}\left(x^{\prime}, y^{\prime}\right) d \mu^{\prime}\left(y^{\prime}\right)=0
$$

so in place of (10.3) we may write

$$
\int_{\widetilde{K}^{\prime}} \int_{0}^{\infty} \int_{\widetilde{K}^{\prime}} u^{\prime}\left(x^{\prime}\right)\left(u^{\prime}\left(x^{\prime}\right)-u^{\prime}\left(y^{\prime}\right)\right) \frac{d}{d t} \widetilde{p}_{t}^{\prime}\left(x^{\prime}, y^{\prime}\right) t^{-\frac{2 s}{d^{\prime}+1}} d \mu^{\prime}\left(y^{\prime}\right) d t d \mu^{\prime}\left(x^{\prime}\right)
$$

and by symmetry

$$
\frac{1}{2} \int_{\widetilde{K}^{\prime}} \int_{0}^{\infty} \int_{\widetilde{K}^{\prime}}\left|u^{\prime}\left(x^{\prime}\right)-u^{\prime}\left(y^{\prime}\right)\right|^{2} \frac{d}{d t} \widetilde{p}_{t}^{\prime}\left(x^{\prime}, y^{\prime}\right) t^{-\frac{2 s}{d^{\prime}+1}} d \mu^{\prime}\left(y^{\prime}\right) d t d \mu^{\prime}\left(x^{\prime}\right) .
$$

Integrating by parts yields

$$
\int_{0}^{\infty} \frac{d}{d t} \widetilde{p}_{t}^{\prime}\left(x^{\prime}, y^{\prime}\right) t^{-\frac{2 s}{d^{\prime}+1}} d t=\frac{2 s}{d^{\prime}+1} \int_{0}^{\infty} \widetilde{p}_{t}^{\prime}\left(x^{\prime}, y^{\prime}\right) t^{-\frac{2 s}{d^{\prime}+1}-1} d t
$$

Using the two-sided heat kernel estimate we see that $\|u\|_{L_{s}^{2}\left(\widetilde{K}^{\prime}\right)}^{2}$ is equivalent to

$$
\int_{\widetilde{K}^{\prime}} \int_{\widetilde{K}^{\prime}} \frac{\left|u^{\prime}\left(x^{\prime}\right)-u^{\prime}\left(y^{\prime}\right)\right|^{2}}{d^{\prime}\left(x^{\prime}, y^{\prime}\right)^{d^{\prime}+2 s}} d \mu^{\prime}\left(x^{\prime}\right) d \mu^{\prime}\left(y^{\prime}\right) \text {. }
$$


We can obtain a similar estimate on $\widetilde{K}$. Again we require $s<\left(d^{\prime}+1\right) / 2$. Reasoning as before we obtain the analog of (10.4), namely

$$
\|u\|_{L_{s}^{2}(\widetilde{K})}^{2}=\frac{1}{2} \int_{\widetilde{K}} \int_{0}^{\infty} \int_{\widetilde{K}}|u(x)-u(y)|^{2} \frac{d}{d t} \widetilde{p}_{t}(x, y) t^{-\frac{2 s}{d^{\prime}+1}} d \mu(x) d t d \mu(y) .
$$

The rest of the argument is the same, showing that $\|u\|_{L_{s}^{2}(\widetilde{K})}^{2}$ is equivalent to

$$
\int_{\widetilde{K}} \int_{\widetilde{K}} \frac{|u(x)-u(y)|^{2}}{d(x, y)^{2 d^{\prime}+2 s}} d \mu(x) d \mu(y) .
$$

Note that the condition $s<\left(d^{\prime}+1\right) / 2$ rules out using $(10.8)$ for $\operatorname{dom} \widetilde{\mathcal{E}}$ and $\operatorname{dom}_{L^{2}} \widetilde{\Delta}$. On the other hand (10.6) does apply to the cases $s=1 / 2$ and $s=d^{\prime} / 2$ that arise as traces of dom $\widetilde{\mathcal{E}}$ and normal derivatives of $\operatorname{dom}_{L^{2}} \widetilde{\Delta}$, but not to the case $s=\frac{d^{\prime}}{2}+1$ that arises from traces of $\operatorname{dom}_{L^{2}} \widetilde{\Delta}$.

\section{Hypoellipticity}

Hypoellipticity means that solutions of elliptic PDE are smooth in the interior of the domain where the PDE holds. We want to show that the same principle holds for harmonic functions on $K$. Before we can do this we need to define precisely what "smooth" means in this context, and in particular we need to define precisely the operators $\Delta^{\prime}$ and $\Delta^{\prime \prime}$ on $K$.

Definition 11.1. Let $u$ and $f$ be continuous on $K$. We say $u \in \operatorname{dom} \Delta^{\prime}$ and $\Delta^{\prime} u=f$ if for each fixed $x^{\prime \prime}, \Delta^{\prime} u\left(\cdot, x^{\prime \prime}\right)=f\left(\cdot, x^{\prime \prime}\right)$ (and similarly for $\Delta^{\prime \prime}$ ). Similarly, if $C$ is a simple subset of $K$ we define dom $\left.\Delta^{\prime}\right|_{C}$ and $\Delta^{\prime} u=f$ on $C$.

Note that if $u \in \operatorname{dom} \Delta^{\prime}$ and $u \in \operatorname{dom} \Delta^{\prime \prime}$, then $u \in \operatorname{dom} \Delta$ and $\Delta u=\Delta^{\prime} u+\Delta^{\prime \prime} u$.

As in ordinary calculus, these operators commute.

Lemma 11.2. Suppose $u \in \operatorname{dom} \Delta^{\prime}$ and $\Delta^{\prime} u \in \operatorname{dom} \Delta^{\prime \prime}$. Then $u \in \operatorname{dom} \Delta^{\prime \prime}, \Delta^{\prime \prime} u \in$ dom $\Delta^{\prime}$ and $\Delta^{\prime \prime} \Delta^{\prime} u=\Delta^{\prime} \Delta^{\prime \prime} u$. A similar result holds on any simple subset $C$.

Proof. We use the Green's function characterization of $\Delta^{\prime}$ on $K^{\prime}$, so $\Delta^{\prime} u^{\prime}\left(x^{\prime}\right)=$ $f^{\prime}\left(x^{\prime}\right)$ if and only if

$$
u^{\prime}\left(x^{\prime}\right)=\int_{K^{\prime}} G_{D}^{\prime}\left(x^{\prime}, y^{\prime}\right) f^{\prime}\left(y^{\prime}\right) d \mu^{\prime}\left(y^{\prime}\right)+h^{\prime}\left(x^{\prime}\right)
$$

for some harmonic function $h^{\prime}$. More precisely, $h^{\prime}$ is the harmonic function satisfying $h^{\prime}\left(q_{i}^{\prime}\right)=u^{\prime}\left(q_{i}^{\prime}\right)$.

Applying this to the function $u\left(\cdot, x^{\prime \prime}\right)$ for each fixed $x^{\prime \prime}$ yields

$$
u\left(x^{\prime}, x^{\prime \prime}\right)=\int_{K^{\prime}} G_{D}^{\prime}\left(x^{\prime}, y^{\prime}\right) f\left(y^{\prime}, x^{\prime \prime}\right) d \mu^{\prime}\left(y^{\prime}\right)+h^{\prime}\left(x^{\prime}, x^{\prime \prime}\right)
$$

if $\Delta^{\prime} u=f$, where $h^{\prime}$ is continuous on $K$ and $\Delta^{\prime} h^{\prime}\left(\cdot, x^{\prime \prime}\right)=0$ for each fixed $x^{\prime \prime}$. Now $f \in \operatorname{dom} \Delta^{\prime \prime}$ with say $\Delta^{\prime \prime} f=g$, so the analog of (11.1) yields

$$
f\left(y^{\prime}, x^{\prime \prime}\right)=\int_{K^{\prime \prime}} G_{D}^{\prime \prime}\left(x^{\prime \prime}, y^{\prime \prime}\right) g\left(y^{\prime}, y^{\prime \prime}\right) d \mu^{\prime \prime}\left(y^{\prime \prime}\right)+H\left(y^{\prime}, x^{\prime \prime}\right),
$$

where $\Delta^{\prime \prime} H\left(y^{\prime}, x^{\prime \prime}\right)=0$ for each $y^{\prime}$. Substituting (11.3) in (11.2) yields

$$
\begin{gathered}
u\left(x^{\prime}, x^{\prime \prime}\right)=\int_{K^{\prime}} \int_{K^{\prime \prime}} G_{D}^{\prime}\left(x^{\prime}, y^{\prime}\right) G_{D}^{\prime \prime}\left(x^{\prime \prime}, y^{\prime \prime}\right) g\left(y^{\prime}, y^{\prime \prime}\right) d \mu^{\prime \prime}\left(y^{\prime \prime}\right) d \mu^{\prime}\left(y^{\prime}\right) \\
+h^{\prime}\left(x^{\prime}, x^{\prime \prime}\right)+h^{\prime \prime}\left(x^{\prime}, x^{\prime \prime}\right),
\end{gathered}
$$


where $h^{\prime \prime}\left(x^{\prime}, x^{\prime \prime}\right)=\int G_{D}^{\prime}\left(x^{\prime}, y^{\prime}\right) H^{\prime \prime}\left(y^{\prime}, x^{\prime \prime}\right) d \mu^{\prime}\left(y^{\prime}\right)$ is continuous on $K$ and satisfies $\Delta^{\prime \prime} h^{\prime \prime}\left(x^{\prime}, x^{\prime \prime}\right)=0$. Now we may interchange integrals in (11.4) because the Green's functions are continuous, and work backwards to conclude $\Delta^{\prime} \Delta^{\prime \prime} u=g$ also.

The argument is similar for the restriction to a simple subset $C$. Note that each section of $C$ is a simple subset of $K^{\prime}$ or $K^{\prime \prime}$, and there exists a continuous Green's function for simple subsets of $K^{\prime}$ (this follows from [Ki4]).

Definition 11.3. A function $u$ is said to be smooth on $K$ (or any simple subset of $K$ or even $\widetilde{K}$ ) if $\left(\Delta^{\prime}\right)^{j}\left(\Delta^{\prime \prime}\right)^{k} u$ is continuous for every $j$ and $k$. More generally, if $\Omega$ is any domain in $K, u$ is said to be smooth on $\Omega$ if the restriction of $u$ to every simple subset contained in $\Omega$ is smooth.

Theorem 11.4. Assume (8.3) and its local analog. Let $u$ be a harmonic function of finite energy on $K$. Then $u$ is smooth on the interior of $K$. More generally, if $u$ is harmonic on an open set $\Omega$, then $u$ is smooth on $\Omega$.

Proof. Let $C=C^{\prime} \times C^{\prime \prime}$ be a simple subset not intersecting $\partial K$. By Theorem 8.2, u is continuous on $C$. By subtracting a pluriharmonic function we may arrange that $u$ vanish on the distinguished boundary of $C$. Then $u$ may be written as a finite sum of terms of the local version of (8.19), where the coefficients $\left\{a_{j}\right\} \in \ell^{2}$. The estimate (8.25) shows that we may apply the operators $\Delta^{\prime}$ and $\Delta^{\prime \prime}$ any number of times to (8.19) and still obtain a uniformly convergent series on any simple subset in the interior of $C$ (these operators produce factors of $\pm \lambda_{j, D}^{\prime}$ ).

For the local result we just have to start with a simple subset $C$ of the form $C^{\prime} \times C^{\prime \prime}$ in $\Omega$, and repeat the same argument, noting that every point in $\Omega$ lies in such a simple subset.

We expect that the same conclusion holds if instead of assuming $u$ is harmonic we merely assume $\Delta u=f$ where $f$ is smooth.

Corollary 11.5 (Local Gauss-Green formula). Let $u \in \operatorname{dom}_{L^{2}} \Delta$ and let $C$ be any simple subset that does not intersect the boundary of $K$. Then the conclusions of Theorem 5.4 hold.

Proof. We have $\Delta u=f$ on $K$ with $f \in L^{2}(K)$. Write $u=\left(u+G_{D}(f)\right)-G_{D}(f)$ for $G_{D}(f)(x)=\int_{K} G_{D}(x, y) f(y) d \mu(y)$. Then $G_{D}(f)$ extends by OR to a function in $\operatorname{dom}_{L^{2}} \widetilde{\Delta}$ where Theorem 5.4 holds. But $u+G_{D} f$ is harmonic in $K$, hence smooth on $C$. It is easy to see that Theorem 5.4 holds for smooth functions.

On $\widetilde{K}$, it is easy to see using Theorem 5.1 that a function $u$ is smooth if and only if it is in $\operatorname{dom}(\widetilde{\Delta})^{\infty}$, and this holds if and only if $a_{j k}(u)$ is rapidly decreasing. Of course nothing like this holds on $K$ or locally.

\section{ACKNOWLEDGMENTS}

We are grateful to Alexander Bendikov, Takashi Kumagai, Gregory Lawler, Larry Payne, and Al Schatz for helpful comments.

\section{REFERENCES}

[B] G. Barbatis, Explicit estimates on the fundamental solution of higher-order parabolic equations with measurable coefficients, J. Diff. Eq. 174 (2001), 442-463. MR 1846743 (2002i:35002) 
[BD] G. Barbatis and E. B. Davies, Sharp bounds on heat kernels of higher order uniform operators, J. Operator Theory 36 (1996), 179-198. MR 1417193 (97k:35105)

[Ba] M. Barlow, Diffusion on fractals, Lecture Notes Math., vol. 1690, Springer, 1998. MR 1668115 (2000a:60148)

[BP] M. Barlow and E. Perkins, Brownian motion on the Sierpinski gasket, Probab. Theory Related Fields 79 (1988), 543-623. MR 0966175 (89g:60241)

[BST] O. Ben-Bassat, R. Strichartz and A. Teplyaev, What is not in the domain of the Laplacian on Sierpinski gasket type fractals, J. of Functional Analysis 166 (1999), 197-217. MR 1707752 (2001e:31016)

[BSSY] N. Ben-Gal, A. Shaw-Krauss, R. Strichartz and C. Young, Calculus on the Sierpinski gasket II: point singularities, eigenfunctions, and normal derivatives of the heat kernel, preprint.

[Be] C. Berg, Potential theory on the infinite dimensional torus, Inv. Math. 32 (1976), 49-100. MR 0402093 (53:5915)

[BrP] J. H. Bramble and L.E. Payne, Bounds for the first derivatives of Green's function, Atti Accad. Naz. Lincei. Rend. Cl. Sci. Fis. Mat. Natur (8) 42 (1967), 604-610. MR $0224850(37: 449)$

[C] T. Coulhon, Off-diagonal heat kernel lower bounds without Poincaré, J. London Math. Soc. (2) 68 (2003), 795-816. MR 2010012

[CDS] K. Coletta, K. Dias and R. Strichartz, Numerical analysis on the Sierpinski gasket, with applications to Schrödinger equations, wave equation, and Gibbs' phenomenon, Fractals (to appear).

[DSV] K. Dalrymple, R. Strichartz and J. Vinson, Fractal differential equations on the Sierpinski gasket, J. Fourier Anal. Appl. 5 (1999), 203-284. MR 1683211 (2000k:31016)

[DOS] X. T. Duong, E. M. Ouhabaz and A. Sikora, Plancherel type estimates and sharp spectral multipliers, J. Funct. Anal. 196 (2002), 443-485. MR 1943098 (2003k:43012)

[FS] M. Fukushima and T. Shima, On a spectral analysis for the Sierpinski gasket, Potential Anal. 1 (1992), 1-35. MR 1245223 (95b:31009)

[GHL] A. Grigor'yan, Jiaxin Hu and Ka-Sing Lau, Heat kernels on metric measure spaces and an application to semilinear elliptic equations, Trans. Amer. Math. Soc. 255 (2003), 2065-2095. MR 1953538 (2003j:60103)

[HK] B. Hambly and T. Kumagai, Transition density estimates for diffusion processes on post critically finite self-similar fractals, Proc. London Math. Soc. 78 (3) (1999), 431-458. MR 1665249 (99m:60118)

[KZ] S. Kasuoka and X. Y. Zhou, Dirichlet forms on fractals: Poincaré constant and resistance, Probab. Theory Related Fields 93 (1992), 169-196. MR 1176724 (94e:60069)

[Ki1] J. Kigami, A harmonic calculus on the Sierpinski spaces, Japan J. Appl. Math. 8 (1989), 259-290. MR 1001286 (91g:31005)

[K2] J. Kigami, Harmonic calculus on p.c.f. self-similar sets, Trans. Amer. Math. Soc. 335 (1993), 721-755. MR 1076617 (93d:39008)

[Ki3] J. Kigami, Analysis on Fractals, Cambridge University Press, New York, 2001. MR 1840042 (2002c:28015)

[Ki4] J. Kigami, Harmonic analysis for resistance forms, J. Funct. Anal. 204 (2003), 399-444. MR 2017320

[M] V. Metz, Renormalization contracts on nested fractals, J. Reine Angew. Math. 480 (1996), 161-175. MR 1420562 (98b:31007)

[OSS] R. Oberlin, B. Street and R. Strichartz, Sampling on the Sierpinski gasket, Experimental Math. 12 (2003), 403-418.

[P] R. Peirone, Convergence and uniqueness problems for Dirichlet forms on fractals, Boll. Unione Mat. Ital. Sez. B Artic. Ric. Mat. (8) 3 (2000), 431-460. MR 1769995 (2001i:31016)

[Sa] C. Sabot, Existence and uniqueness of diffusions on finitely ramified self-similar fractals, Ann. Sci. Écoel Norm. Sup (4) 30 (1997), 605-673. MR 1474807 (98h:60118)

[SST] J. Stanley, R. Strichartz and A. Teplayev, Energy partition on fractals, Indiana Univ. Math. Journal 52 (2003), 133-156. MR 1970024 (2004a:31006)

[SB] L. N. Slobodeckii and V. M. Babic, On bounds for the Dirichlet integrals (Russian), Doklady Akad. Nauk. S.S.S.R. 106 (1956), 604-606. MR 0076886 (17:959d) 
[S1] R. Strichartz, Multipliers on fractional Sobolev spaces, J. Math. Mech. 16 (1967), 10311060. MR 0215084 (35:5927)

[S2] R. Strichartz, Some properties of Laplacians on fractals, J. Funct. Anal. 164 (1999), 181-208. MR 1695571 (2000f:35032)

[S3] R. Strichartz, Analysis on fractals, Notices Amer. Math. Soc. 46 (1999), 1199-1208. MR 1715511 (2000i:58035)

[S4] R. Strichartz, The Laplacian on the Sierpinski gasket via the method of averages, Pac. J. Math. 201 (2001), 241-256. MR 1867899 (2003f:35056)

[S5] R. Strichartz, Function spaces on fractals, J. Funct. Anal. 198 (2003), 43-83. MR 1962353 (2003m:46058)

[S6] R. Strichartz, Fractafolds based on the Sierpinski gasket and their spectra, Trans. Amer. Math. Soc. 355 (2003), 4019-4043. MR 1990573 (2004b:28013)

[SU] R. Strichartz and M. Usher, Splines on fractals, Math. Proc. Cambridge Phil. Soc. 129 (2000), 331. MR 1765920 (2001c:28016)

Department of Mathematics, Malott Hall, Cornell University, Ithaca, New York 14853

E-mail address: str@math.cornell.edu 